\title{
Proto-Ong-Be Initials and Finals
}

\author{
Yen-ling Chen \\ University of Hawai'i at Mānoa/ Academia Sinica \\ yenling@hawaii.edu
}

\begin{abstract}
This study is the first to provide a bottom-up reconstruction of Proto-Ong-Be initials and finals using the comparative method. Thirty-four initials (labelled with tone series) and nine finals that can be reconstructed with confidence are postulated. This paper shows that the early voicing contrast associated with initials cannot be directly reconstructed based on the internal evidence, although the loss of the contrast is compensated for at the suprasegmental level. This study also demonstrates that Proto-Ong-Be finals remain intact in modern Ong-Be varieties. In addition, this paper serves as a testing ground and a demonstration for doing linguistic reconstruction from different angles.
\end{abstract}

\section{Keywords}

Ong-Be - initials - finals - reconstruction - Kra-Dai

\section{Introduction}

\subsection{Prospectus}

The objective of this study is to provide a bottom-up reconstruction of Proto-Ong-Be consonants using the comparative method. The postulated reconstruction is based on first-hand lexical materials gathered in the field in 2015 and 2017. This study also compares different reconstructions of Proto-Ong-Be consonants and demonstrates how they reflect the phonological system of Ong-Be at different times, which helps us better understand sound change in Ong-Be.

The classification of Ong-Be within the Kra-Dai language family is beyond the scope of this article and will not be addressed here because it requires (1) a full reconstruction of the Proto-Ong-Be phonological system, not just its consonantal system, and (2) reconstructions above the Proto-Ong-Be level to distinguish shared innovations from retentions.

\section{$1.2 \quad$ Background}

Ong-Be (Iso 639-3: onb), a.k.a. Lingaohua 臨高話, is a subgroup of Kra-Dai, composed of two languages spoken in the regions located to the west of the Nandu River (南渡江), northwestern Hainan (海南), China. One is found in scattered locations in Haikou City (海口市) and northeastern Chengmai County (澄邁縣), and the other is spoken in northwestern Chengmai County, Lingao County (臨高縣) and the nearby regions of Danzhou City (儋州市) (see Map 1). Although both languages are commonly 


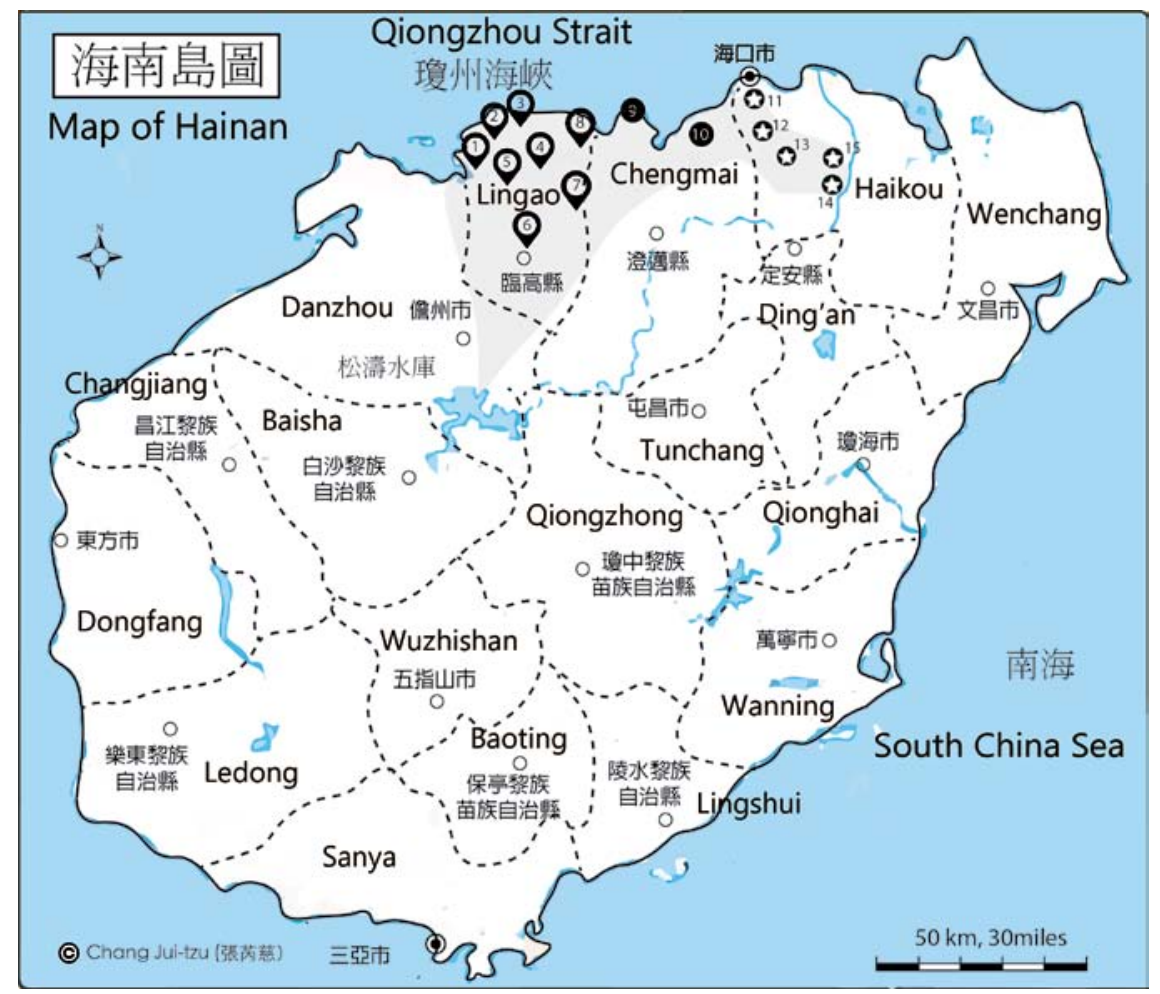

MAP $1 \quad$ Map of Hainan ${ }^{1,2}$

referred to as Ong-Be/Lingaohua, they are mutually unintelligible, and each has its own sub-varieties. Chen (2018) proposes the terms, Eastern Ong-Be and Western Ong-Be, for the two languages, corresponding to their geographical distributions.

The term Ong-Be $/ \mathrm{Raj}^{3} \mathrm{be}^{3} /$ was first recorded by the French Missionary F.M. Savina based on the Changliu (長流) variety. It is an autonym which literally means "prefix $x_{\text {[human] }}$ village," hence "village people." "Ong-Be" / $\mathrm{Pa \eta}^{3} \mathrm{Ge}^{3} /$ in the Changliu variety corresponds to $/ \mathrm{Pa \eta}^{3} \mathrm{vo}^{3} /$ in other Ong-Be varieties. The term Ong-Be is commonly used in materials published outside China, but the term Lingaohua "Lingao language" is mostly used in Chinese scholarship, given that Lingao County has the most Ong-Be speaking population. Note that in this study, Ong-Be is used to refer to the entire branch, rather than a particular variety.

1 Map 1 is illustrated by Chang Jui-tzu, which bases its geographic frame on a map provided by Holiday China Tour (n.d.) and its administrative boundary on the 2017 version of the Hainan Map published by Hainan Administration of Surveying Mapping and Geoinformation. The varieties spoken in Lingao County, Chengmai County, and Haikou City (including former Qiongshan County) are marked with a black number in a white circle (1-8), a white number in a black circle (9, 10), and a white star in a black background (11-15), respectively.

2 The shaded area on Map 1 indicates the Ong-Be speaking regions, which are mostly plains. In addition to Ong-Be, there are several Kra-Dai languages, Sinitic languages, a Mienic language, and an Austronesian language spoken on the island. See Language Atlas of China (中國語言地圖集) compiled by The Institute of Linguistics (Chinese Academy of Social Sciences) et al. (2012) for more information. 
A list of the Ong-Be varieties surveyed is shown in Table 1.

TABLE 1 The locations surveyed

\begin{tabular}{|l|l|l|}
\hline$\#$ & Townships & Sources \\
\hline 1 & 新盈 Xinying & Hashimoto (1980); my fieldwork [Cangmi village/倉米村] \\
\hline 2 & 美良 Meiliang & my fieldwork [Kundian village/昆殿村] \\
\hline 3 & 東英 Dongying & Zhang et al. (1985) \\
\hline 4 & 臨城 Lincheng & Jeremiassen (1893); ; Liang \& Zhang (1997) \\
\hline 5 & 波蓮 Bolian & Liu (200o) \\
\hline 6 & 加來 Jialai & my fieldwork [Langguan village/郎貫村] \\
\hline 7 & 馬裊 Maniao & my fieldwork [Yangda village/洋大村; Daozao village/道灶村] \\
\hline 8 & 皇桐 Huangtong & my fieldwork [Wenxian village/文顯村] \\
\hline 9 & 橋頭 Qiaotou & my fieldwork [Linshigang/林詩港] \\
\hline 10 & 老城 Laocheng & Zhang et al. (1985) \\
\hline 11 & 長流 Changliu & Savina (1965); Xin (2008); my fieldwork [Qionghua village/瓊華村] \\
\hline 12 & 石山 Shishan & Parker (1892);Xin (2011) \\
\hline 13 & 永興 Yongxing & my fieldwork [Nandao village/南道村] \\
\hline 14 & 龍塘 Longtang & $\begin{array}{l}\text { Liang \& Zhang (1997); my fieldwork [Renhe village/仁何村;Wencai village/ } \\
\text { 文彩村] }\end{array}$ \\
\hline 15 & 龍橋 Longqiao & Zhang et al. (1985) \\
\hline
\end{tabular}

a Jeremiassen did not specify which variety he investigated. Based on the phonology and the lexicon, Xin (2007: 123) suggests that it is a variety closely related to Lincheng and/or the Ong-Be varieties spoken in central-south Lingao.

Chinese scholars tend to consider the Kra-Dai language family, to which Ong-Be belongs, as part of Sino-Tibetan. However, the resemblances between Kra-Dai and Sinitic have been ascribed to typological factors and lexical borrowing by most non-Chinese linguists, as there is no clear evidence for a genetic relationship between Kra-Dai and Tibeto-Burman languages. ${ }^{3}$ In addition, the Sinitic words found in Kam-Sui and Tai, which are in close contact with Chinese, are often absent in Hlai or Kra, both of which belong to the Kra-Dai branches which have had little contact with Chinese. Those Sinitic words are not reconstructible to Proto-Kra-Dai, and should be regarded as loans, not cognates between Kra-Dai and Sinitic (Ostapirat 2016). Considering the basic vocabulary, there is no doubt that although Ong-Be speakers are classified as Han Chinese by the government in terms of nationality, their language is Kra-Dai, not Sino-Tibetan, cf. Zhang et al. (1985: 8-10), and Liang \& Zhang (1997:1).

3 The Sino-Tibetan language family is traditionally, although not uncontroversially, divided into the Sinitic branch and the Tibeto-Burman branch. If Kra-Dai were genetically related to Sino-Tibetan languages, but was not a subgroup within Sinitic, we would expect to see cognates shared exclusively between Kra-Dai and Tibeto-Burman, but this is not the case. The lexical evidence certain scholars claim to support the genetic relationship between Kra-Dai and Sinitic is best attributed to borrowing. 


\section{Hansell (1988)}

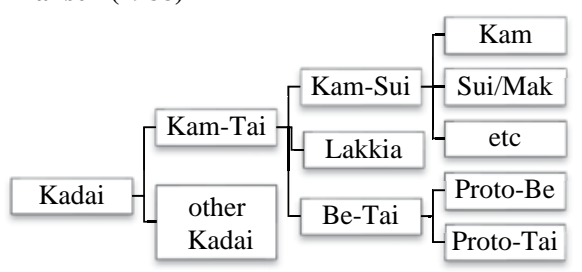

\section{Edmondson \&}

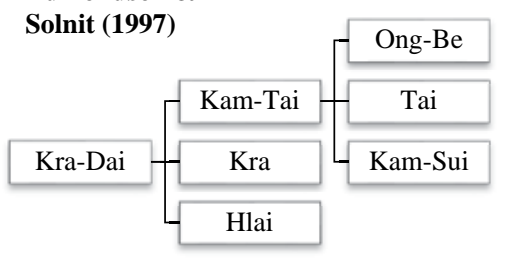

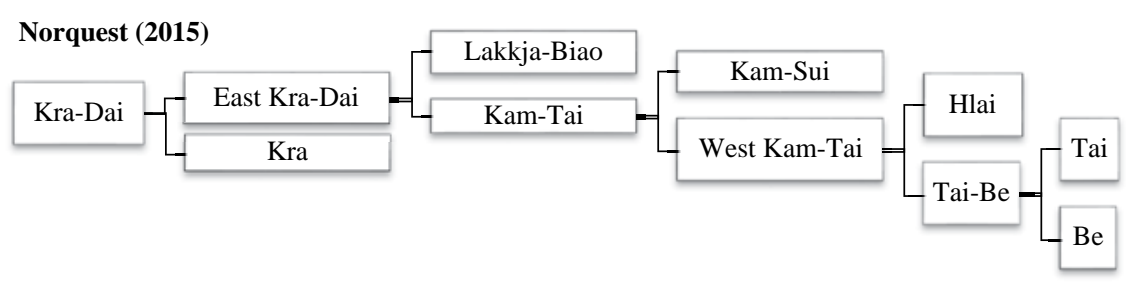

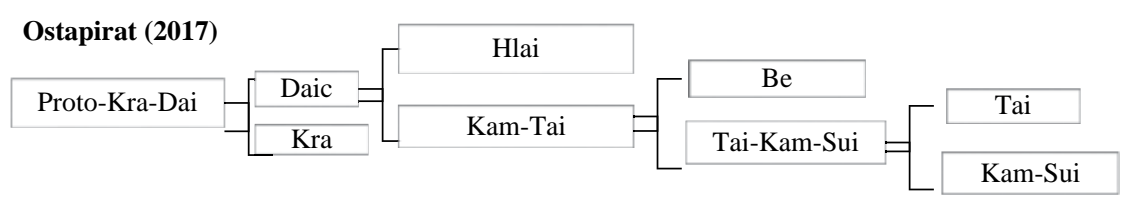

FIGURE 1 The Kra-Dai language family

Within the Kra-Dai language family, scholars have divergent opinions regarding subgrouping, as illustrated in Figure 1. Ong-Be is traditionally placed under Kam-Tai (Hansell 1988; Edmondson \& Solnit 1997; Ostapirat 2000; among others) although details concerning the internal subgrouping of Kam-Tai have never been presented. Hansell (1988) proposes that Ong-Be and Tai form a Be-Tai subgroup under the Kam-Tai node. Liang \& Zhang (1996: 69) classifies Ong-Be as a Tai subgroup, whereas Xin (2006) considers it an independent, non-Tai subgroup closely related to Southern Zhuang. Norquest (2015: 3) places Ong-Be with Tai to form a Tai-Be branch of his West Kam-Tai subgroup, but Norquest (2007) regards Ong-Be as a subgroup under the Be-Tai node within his Southern Kra-Dai branch. On the other hand, Ostapirat (2005a) considers Ong-Be a primary branch in the Kra-Dai language family, whereas Ostapirat (2017) places Ong-Be under the Kam-Tai node.

Ong-Be has a Sino-Ong-Be stratum composed of multiple layers in addition to its native Kra-Dai lexicon, which has made its linguistic status controversial. Stübel (1937), Woon (1979) and Liu (2000: 9) even consider Ong-Be a mixed Sinitic language. Nevertheless, Ong-Be is unlikely to be a mixed language because a mixed language, which generally arises from widespread bi- or multilingualism in its source languages, is more likely to serve as an in-group identity marker than a lingua franca (see Meakins 2013). On the contrary, Ong-Be speakers do not share a single group identity. To our knowledge, monolingual speakers of Ong-Be were common in the past, and are not uncommon now among people above 65, especially those who are uneducated or rarely come into contact with non-Ong-Be speakers. Ong-Be had been the lingua franca only in Lingao County, where everyone, regardless of their linguistic background, had to learn to speak Ong-Be for communicative purposes. 
According to Xin (2007), the earliest known documents on Ong-Be date to the late $19^{\text {th }}$ century, namely Parker (1892) on Shishan and Jeremiassen (1893) on a variety of Lingao. Synchronic studies of Ong-Be include Savina (1965) on Changliu, Hashimoto (1980) on Xinying, Zhang et al. (1985) on Dongying, Laocheng, and Longqiao, Liang \& Zhang (1997) on Lincheng and Longtang, Liu (2000) on Bolian, Xin (2008) on Changliu, and Xin (2011) on Shishan. The remainder of this section reviews several diachronic studies of Ong-Be.

\subsection{Hansell (1988)}

Hansell (1988) presents the most systematic comparison between Ong-Be (mostly based on Hashimoto 1980) and Proto-Tai (based on Li 1977) regarding initials and tones. Several sound change routes have been suggested. For example, he proposed that $p^{1}$ - in 'rain' and ' millet' and $v^{1}$ - in 'dream' in Ong-Be were

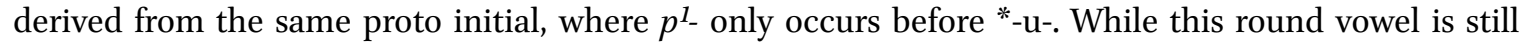
reconstructible in Tai, this labialization environment is not observed in today's Ong-Be (Hansell 1988: 269). Ostapirat (2005b: 277-278), on the other hand, suggests that these two initials in Ong-Be were conditioned by different Proto-Kra-Dai presyllablic onsets.

Hansell (1988: 268-270) compares the Ong-Be data with Sui data and notes that $t^{1}$ - and $t^{2}$ - in Ong-Be are the result of palatalization, which is a shared retention of both Ong-Be and Sui (also see Solnit 1982: 224 and Ostapirat 2005b: 281-283). Nevertheless, he found that neither labialization nor palatalization can be reconstructed in Proto-Ong-Be based solely on the internal evidence.

Hansell (1988: 280-285) concludes that shared lexical innovations show that Ong-Be is more closely related to Tai than to Kam-Sui or Hlai. No shared phonological innovations between Ong-Be and Tai were presented, however. He argues that Tai and Ong-Be were derived from a common ancestor he calls 'Be-Tai' which is a sister language of Kam-Sui.

\section{$2.2 \quad$ Ostapirat $(1998 ; 2005 b)$}

Ostapirat (1998) is the first to demonstrate that Jizhao Haihua (吉兆海話), a language spoken in Jizhao village of Tanba Town (覃巴鎮), Wuchuan City (吳川市) in southwestern Guangdong, contains Ong-Be related elements that are confined to basic vocabulary.

Ostapirat suggests that it is reasonable to postulate that 'the Jízhào were rather a Bê group, which has become assimilated to Chinese, but somehow kept remnants of their former mother tongue in a few basic words' (p. 341) However, because there is no evidence indicating the nature of the contact that the Jizhao and the Ong-Be had, it is open for discussion whether Jizhao Haihua speakers were descendants of a larger indigenous people of Leizhou who once spoke a language resembling Ong-Be, but have been assimilated by the Chinese-speaking populations.

Ostapirat (2005b) examines the Ong-Be obstruents from a Kra-Dai perspective, and shows how residuals of Proto-Kra-Dai are reflected in today's Ong-Be. He concludes that 'early Kd obstruent initial and medial consonants have shuffled into a Proto-Be system that chiefly contrasts voiceless and voiced initials. These initials were in more recent history devoiced, implosivized, and had given up their voicing contrast for tonal distinction.' (pp. 289-290). A detailed comparison between Ostapirat (2005b) and the reconstruction proposed in this study is presented in $\$ 5.3$. The major difference lies in the reconstruction of an early voicing contrast in initials. 
2.3 Norquest (2007)

Norquest (2007: 249-306) reconstructs lexical items for Proto-Ong-Be, although he does not explicitly state a phoneme inventory for Proto-Ong-Be. Based on his reconstructed vocabulary, Norquest's ProtoOng-Be consonant inventory is presented in Table 2.

TABLE $2 \quad$ Norquest's Proto-Ong-Be consonant inventory

\begin{tabular}{|c|c|c|c|c|c|c|}
\hline & bilabial & labiodental & alveolar & (alveo)palatal & velar & glottal \\
\hline stops & ${ }^{*} \mathrm{p} \quad * \mathrm{~b}$ & & ${ }^{*} \mathrm{t} \quad * \mathrm{~d}$ & ${ }^{*} \mathrm{c}$ & ${ }^{*} \mathrm{k} \quad * \mathrm{~g}$ & *? \\
\hline nasals & ${ }^{*} \mathrm{~m}$ & & *n & ${ }^{*} \mathrm{n}$ & $* \eta$ & \\
\hline fricatives & & ${ }^{*} \mathrm{f} \quad{ }^{*} \mathrm{v}$ & ${ }^{*} \mathrm{~S} \quad{ }^{*} \mathrm{z}$ & ${ }^{*} 6$ & ${ }^{*} \mathrm{x} \quad{ }^{*} \mathrm{\gamma}$ & ${ }^{*} \mathrm{~h} \quad * \mathrm{~h}$ \\
\hline trills & & & *r & & & \\
\hline laterals & & & *l & & & \\
\hline approximants & ${ }^{*} \mathrm{~W}$ & & & ${ }^{*} \mathrm{j}$ & & \\
\hline
\end{tabular}

Norquest reconstructs a voicing contrast, and states that voiceless sonorants resulted from consonant clusters in which the first segment was voiceless. He does not reconstruct any affricates, but does reconstruct labiodental and velar fricatives. In his system, ${ }^{*}$ c occurs only in coda position, and ${ }^{*} \mathrm{j}$ never occurs syllable-initially. Norquest's canonical syllable structure for Proto-Ong-Be is $C(C) V(C)^{4}$ in which the medial (the second segment in a cluster) can be either *-w- or *-j-. Because Norquest did not provide an explanation of his notation regarding Proto-Ong-Be, it remains unclear if his $p j-, \mid j-, f w-, 6 w$ - should be treated as complex onsets composed of a consonant followed by a glide, or a single palatalized or labialized segment. It is also unclear if the medials belong to onset or rime.

There is a question of whether Norquest's reconstruction should be considered Proto-Ong-Be or pre-Proto-Ong-Be, as he reconstructs sesquisyllables in addition to monosyllables. Chen (2018) shows that a monosyllabic model is sufficient in explaining sound correspondences and changes in Ong-Be. The current study argues that because a voicing contrast in initials has been completely lost in contemporary Ong-Be varieties, consonant clusters and a voicing contrast are not reconstructible.

With respect to the coda, the reconstruction of Proto-Ong-Be *-c cannot be reached without referring to external evidence, since none of today's Ong-Be varieties employ $-c$ as a coda. The reconstruction of Proto-Tai ${ }^{*}$-c, which is a shared retention of Proto-Kra-Dai ${ }^{*}$-c, is grounded in a recurrent $-k$ : $-t$ correspondence between Saek and the rest Tai languages (Pittayaporn 2009: 211-213; Ostapirat 2009). However, regular discrepancies in the coda are not attested in the Ong-Be languages to serve as the ground for reconstructing *-c because today's Ong-Be varieties regularly reflect Proto-Kra-Dai * ${ }^{*}$ as a glottal stop.

\section{$2.4 \quad$ Chen (2015)}

Based on Liang \& Zhang (1997) and Xin (2008; 2011), Chen (2015) proposes a reconstruction for ProtoOng-Be consonants that includes a voicing contrast instead of a tone series (see Table 3). Chen argues that no consonant clusters or sesquisyllabic onsets can be reconstructed, and phonemic aspiration did

$4 \mathrm{C}$ stands for consonant, $\mathrm{V}$ for vowel, and segments in parenthesis are optional. 
not exist in Proto-Ong-Be. While all consonants are allowed to occur in the onset, only *-m, *-n, *-n, *-p, *-t, *-k and *-? can serve as Proto-Ong-Be codas. ${ }^{*}$-j was not included in Chen's system because Chen did not distinguish off-glides from vowels. Chen, following Ostapirat (2005), suggested that fortition and spirantization are the major sound change mechanisms which took place in Ong-Be. The main differences between Chen (2015) and this paper lie in the reconstruction of a voicing contrast and the assignment of phonetic values to proto-phonemes.

TABLE 3 Chen (2015)'s Proto-Ong-Be consonants

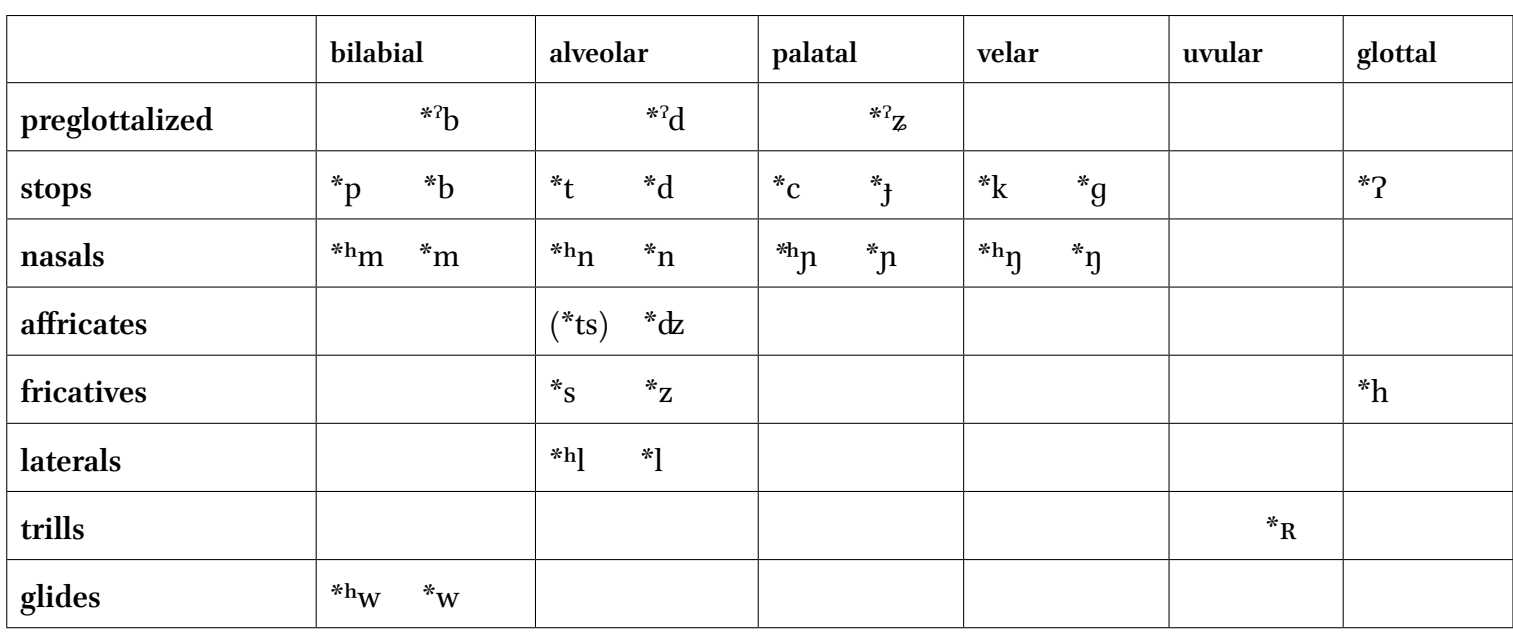

3

\section{The Phonological system of Ong-Be}

This section provides an overview of the phonological system of two Ong-Be languages, where Longtang represents the eastern subgroup and Lincheng represents the western subgroup. According to Chen (2018), the syllable structure of Ong-Be is canonically $\mathrm{CV}(\mathrm{C})^{\mathrm{T}},{ }^{5}$ a pattern which lacks a vowel length distinction. No consonant clusters are allowed in either onset or coda. All consonants, except for approximants, can function as onsets, but only approximants, non-palatal nasals (if any) and voiceless stops occur in codas, and all the stop codas are voiceless and unreleased. Note that this paper focuses on the Kra-Dai lexicon, so the phonology of the Sino-Ong-Be stratum is not addressed.

A modified version of Liang \& Zhang's (1997) phonological system of Longtang and Lincheng is presented in the following section (see Map 1 for their locations). ${ }^{6}$ What I present as $/ 6 /, / d /, / \mathrm{w} /, / \mathrm{j} /, / \mathrm{pf} /$, and $/ \mathrm{kx} /$ in Table 4 are treated as / $\mathrm{bb} /, / \mathrm{Rd} /, / \mathrm{u} /, / \mathrm{i} /, / \mathrm{f} /$ and $/ \mathrm{x} /$ respectively in Liang \& Zhang. The choice of implosives and affricates over preglottalized stops and fricatives is purely notational. ${ }^{7}$ The use of glides in the coda position, rather than vowels, is based on their phonological distribution in which $/ \mathrm{w} /$ and $/ \mathrm{j} /$ do not co-occur with stop and nasal codas.

Tables 4-7 show that there are 16 onsets, nine codas, nine monophthongs, and nine tones in Longtang. Speakers vary in their phonetic realization of the onsets $/ \mathrm{pf} /$ and $/ \mathrm{kx} /$, which can be can be pronounced

$5 \quad \mathrm{~T}$ stands for tone, and diphthongs are treated as single phonemes.

6 The modification is made to facilitate a comparison between my data and the data given in Liang \& Zhang.

7 The phonetic nature of the so-called "preglottalized stops" or "implosives" in Ong-Be remains unclear and requires experimental study. 
as an affricate ([pf] and $[\mathrm{kx}]$ respectively), a fricative $([\mathrm{f}]$ and $[\mathrm{x}])$, or an aspirated stop $\left(\left[\mathrm{p}^{\mathrm{h}}\right]\right.$ and $\left.\left[\mathrm{k}^{\mathrm{h}}\right]\right)$. Only stops, nasals, and approximants are allowed in coda position. All stop codas are voiceless and unreleased and all nasal and approximant codas are voiced. Approximants do not occur in the onset position. In addition, Longtang shows a two-way distinction in both mid vowels and low vowels, and a tonal split in Tone 1 , Tone 7 , and Tone 8.

TABLE 4 The Onset Inventory of Longtang

\begin{tabular}{|c|c|c|c|c|c|}
\hline & bilabial & labiodental & alveolar & velar & glottal \\
\hline stops & 6 & & d & $\mathrm{k}$ & $?$ \\
\hline nasals & $\mathrm{m}$ & & $\mathrm{n}$ & $\eta$ & \\
\hline fricatives & & $\mathrm{v}$ & $\mathrm{s}$ & & $\mathrm{h}$ \\
\hline affricates & $\mathrm{pf}$ & & ts & $\mathrm{kx}$ & \\
\hline laterals & & & l & & \\
\hline
\end{tabular}

TABLE 5 The Coda Inventory of Longtang

\begin{tabular}{|l|l|l|l|l|l|}
\hline & bilabial & alveolar & palatal & velar & glottal \\
\hline stops & $-\mathrm{p}$ & $-\mathrm{t}$ & & $-\mathrm{k}$ & $-\mathrm{P}$ \\
\hline nasal & $-\mathrm{m}$ & $-\mathrm{n}$ & & $-\mathrm{y}$ & \\
\hline approximants & $-\mathrm{w}$ & & $-\mathrm{j}$ & & \\
\hline
\end{tabular}

TABLE 6 Monophthongs in Longtang

\begin{tabular}{|c|c|c|c|}
\hline & Front & Central & Back \\
\hline high & $\mathrm{i}$ & & $\mathrm{u}$ \\
\hline close-mid & $\mathrm{e}$ & ә & o \\
\hline open-mid & $\varepsilon$ & & o \\
\hline low & a & $\mathrm{e}$ & \\
\hline
\end{tabular}

TABLE $7 \quad$ Tones in Longtang

\begin{tabular}{|l|l|l|l|}
\hline Tones & Citation Form & Tones & Citation Form \\
\hline 1 & 13 & 7 & 13 \\
\hline $1^{\prime}$ & 44 & $7^{\prime}$ & 44 \\
\hline 2 & 33 & 8 & 11 \\
\hline 3 & 24 & $8^{\prime}$ & 33 \\
\hline 4 & 11 & & \\
\hline
\end{tabular}


Lincheng has 17 onsets, nine codas, seven monophthongs, and six tones, as presented in Tables 8-11. Lincheng has the same coda inventory as Longtang. As in Longtang, approximants are confined to coda position. Lincheng has an alveo-palatal nasal not observed in Longtang. As for vowels, Lincheng shows a two-way contrast only in mid back vowels. No further tonal splits have been reported.

TABLE 8 The Onset Inventory of Lincheng

\begin{tabular}{|c|c|c|c|c|c|c|}
\hline & bilabial & labiodental & alveolar & alveo-palatal & velar & glottal \\
\hline stops & 6 & & $\mathrm{t} \quad \mathrm{d}$ & & $\mathrm{k}$ & $?$ \\
\hline nasals & $\mathrm{m}$ & & $\mathrm{n}$ & $\mathrm{n}$ & $\eta$ & \\
\hline affricates & & & ts & & & \\
\hline fricatives & & f $\quad$ v & S & Z & $\mathrm{x}$ & $\mathrm{h}$ \\
\hline laterals & & & l & & & \\
\hline
\end{tabular}

TABLE 9 The Coda inventory of Lincheng

\begin{tabular}{|l|l|l|l|l|l|}
\hline & bilabial & alveolar & palatal & velar & glottal \\
\hline stops & $-\mathrm{p}$ & $-\mathrm{t}$ & & $-\mathrm{k}$ & $-\mathrm{P}$ \\
\hline nasal & $-\mathrm{m}$ & $-\mathrm{n}$ & & $-\mathrm{n}$ & \\
\hline approximants & $-\mathrm{w}$ & & $-\mathrm{j}$ & & \\
\hline
\end{tabular}

TABLE 10 Monophthongs in Lincheng

\begin{tabular}{|l|c|l|l|}
\hline & Front & Central & Back \\
\hline high & $\mathrm{i}$ & & $\mathrm{u}$ \\
\hline close-mid & & ə & o \\
\hline open-mid & $\varepsilon$ & & ว \\
\hline low & a & & \\
\hline
\end{tabular}

TABLE 11 Tones in Lincheng

\begin{tabular}{|l|l|l|l|}
\hline Tones & Citation Form & Tones & Citation Form \\
\hline 1 & 213 & 7 & 33 \\
\hline 2 & 55 & 8 & 55 \\
\hline 3 & 33 & & \\
\hline 4 & 21 & & \\
\hline
\end{tabular}


In terms of segmental features, the major differences between these two Ong-Be varieties are: (1) the number of vowels, where Longtang has $/ \mathrm{a} /$ and $/ \mathrm{e} /$, and $/ \mathrm{e} /$ and $/ \varepsilon /$, but Lincheng only has $/ \mathrm{a} /$ and $/ \varepsilon /$; (2) the lenition of voiceless affricates or aspirated stops, where Longtang has /pf/ ([pf] [ $\left.\left[\mathrm{p}^{\mathrm{h}}\right]\right)$ and $/ \mathrm{kx} /$ $\left([\mathrm{kx}] \sim\left[/ \mathrm{k}^{\mathrm{h}}\right]\right)$ corresponding to Lincheng $/ \mathrm{f} /$ and $/ \mathrm{x} /$, and (3) the presence/absence of alveo-palatals, in which Longtang has $/ \mathrm{n} /$ whereas Lincheng shows a $/ \mathrm{n} /-/ \mathrm{n} /$ distinction.

\section{$4 \quad$ Proto-Ong-Be Consonants}

The comparative method can be summarized into three stages: (1) identify regular sound correspondences, (2) set up place-holders, and (3) assign a phonetic value to the place-holders (see Fox 1995 and Campbell 2013 for more details). This study adopts a bottom-up approach to the assignments of the phonetic values to Proto-Ong-Be initials and finals, based upon the phonetic qualities of modern reflexes.

Changliu, Longtang, Huangtong, and Xinying were selected as the foundation for this reconstruction for two reasons: (1) Changliu and Longtang of the eastern subgroup have the most complex phoneme inventory among all the Ong-Be varieties; $(2)$ in the western subgroup, the Huangtong variety preserves the $n$ - $n$ distinction absent in the eastern subgroup, while Xinying preserves $/ \mathrm{p}^{\mathrm{h}} /$ and $/ \mathrm{k}^{\mathrm{h}} /$, which have been lenited to fricatives in most of the western Ong-Be varieties.

Regarding Proto-Ong-Be, this study follows a monosyllable canon $\mathrm{CV}(:)(\mathrm{C})^{\mathrm{T}}$, whereby no consonant clusters are reconstructed and diphthongs are equal to long vowels, both bimoraic. Since all contemporary Ong-Be varieties have lost the early voicing contrast that conditioned the secondary tonal split attested in many Kra-Dai languages, a contrast in voicing in initials is not reconstructed. To put it another way, this study proposes that the loss of a voicing contrast and its associated tonal splits had taken place in pre-Proto-Ong-Be.

\subsection{Proto-initials ${ }^{8}$}

Table 12 provides a summary of my reconstruction of Proto-Ong-Be initials based only on Ong-Be internal evidence. This inventory includes seven stops, four nasals, one affricate, five fricatives, and one lateral. Both fricatives and nasals show an alveolar-alveopalatal distinction. From a typological perspective, this

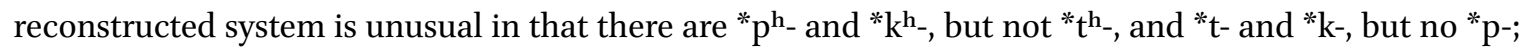
* 6 - but no *p-, and *v- but no *f-. Such distributional gaps can only be explained by bringing in external evidence from beyond Ong-Be in order to see the broader picture, which will be addressed in $\$ 5 \cdot 3$.

TABLE 12 Inventory of Proto-Ong-Be initials

\begin{tabular}{|c|c|c|c|c|c|c|}
\hline & bilabial & labiodental & alveolar & alveo-palatal & velar & glottal \\
\hline implosives & ${ }^{*} 6$ & & ${ }^{*} d$ & & & \\
\hline stops & ${ }^{*} \mathrm{p}^{\mathrm{h}}$ & & $*_{\mathrm{t}}$ & & ${ }^{*} \mathrm{k}^{*} \mathrm{k}^{\mathrm{h}}$ & *? \\
\hline nasals & ${ }^{*} \mathrm{~m}$ & & ${ }^{*} \mathrm{n}$ & ${ }^{*} \mathrm{n}$ & ${ }^{*} \eta$ & \\
\hline
\end{tabular}

8 Some of the Changliu, Longtang, and Xinying data are from Xin (2008), Liang \& Zhang (1997), and Hashimoto (1980), and they are marked respectively as Xin, L\&Z, and HM. LC stands for Lincheng from Liang \& Zhang (1997), and BL for Bolian from Liu (200o). All remaining Ong-Be data are based on my own fieldwork unless otherwise specified. 


\begin{tabular}{|c|c|c|c|c|c|c|}
\hline & bilabial & labiodental & alveolar & alveo-palatal & velar & glottal \\
\hline affricates & & & *ts & & & \\
\hline fricatives & & ${ }^{*} \mathrm{v}$ & *s $\quad$ * $\mathrm{Z}$ & ${ }^{*} \mathrm{z}$ & & ${ }^{*} \mathrm{~h}$ \\
\hline laterals & & & *l & & & \\
\hline
\end{tabular}

4.1.1 Stops

The words listed in Table 13 all begin with a bilabial implosive. Using the comparative method, /6 / is reconstructed for these cognate sets. Although these words agree at the segmental level, they differ in tone.

TABLE $13 \quad 9 * 6$ -

\begin{tabular}{|c|c|c|c|c|c|c|c|}
\hline Chinese & English & CL & $\mathbf{Y X}$ & LT & LSG & нт & $\mathbf{X Y}$ \\
\hline & & $6-$ & $6-$ & $6-$ & $6-$ & $6-$ & $6-$ \\
\hline 魚 & 'fish' & $6 a^{1}$ & $6 a^{1}$ & $6 a^{1}$ & $6 a^{1}$ & $6 a^{1}$ & $6 a^{1}$ \\
\hline 雲 & 'cloud' & $6 a^{4}$ & $6 a^{4}$ & $6 a^{4}$ & $6 a^{4}$ & $6 a^{4}$ & $6 a^{4}$ \\
\hline 水蛭 & 'aquatic leech' & $-6 i \eta^{1}$ & $-6 i \eta^{1}$ & $-6 i y^{\prime}$ & $\operatorname{bin}^{1}$ & $\operatorname{bin}^{1}$ & $\operatorname{bin}^{1}$ \\
\hline 泥 & 'mud' & $6 y^{2}$ & 6on ${ }^{2}$ 'dust' & $6 o \eta^{2}$ & bun $^{2}$ & $6 o \eta^{2}$ & $6 o \eta^{2}$ \\
\hline 乾淨 & 'clean' & $6 a \eta^{3}$ & $6 a y^{3}$ & - & $6 a \eta^{3}$ & $6 a \eta^{3}$ & $6 a \eta^{3}$ \\
\hline$\square 、$ 嘴 & 'mouth' & $6 \mathrm{ak}^{7}$ & $6 \mathrm{ak}^{7}$ & $6 \mathrm{ak}^{7}$ & $6 \mathrm{ak}^{7}$ & $6 \mathrm{ak}^{7}$ & $6 a k^{7}$ \\
\hline 中午 & 'midday' & $6 \mathrm{ak}^{8}$ & $6 \mathrm{ak}^{8}$ & $6 \mathrm{ek}^{8}$ & $6 \mathrm{ak}^{8}$ & - & $6 a k^{8}$ \\
\hline
\end{tabular}

'breakfast'

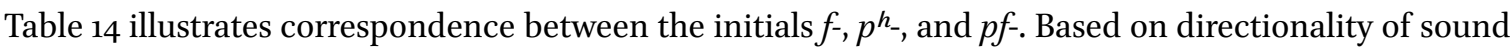
change and the majority-wins principle, I reconstructed a voiceless aspirate ${ }^{*} \mathrm{p}^{\mathrm{h}}$ - for this correspondence. We will see in $\$ 5.3$ that pre-Proto-Ong-Be consonants in medial position underwent spirantization, becoming either aspirated stops or affricates.

TABLE $14 \quad * p^{h_{-}}$

\begin{tabular}{|c|c|c|c|c|c|c|c|}
\hline Chinese & English & CL & $\mathbf{Y X}$ & $\mathbf{L T}$ & LSG & HT & $\mathbf{X Y}$ \\
\hline & & $\mathrm{p}^{\mathrm{h}}$ & $\mathrm{p}^{\mathrm{h}}$ & pf- & f- & $f-$ & $\mathbf{p}^{\mathrm{h}_{-}}$ \\
\hline$天^{b}$ & 'sky' & $\mathrm{p}^{\mathrm{h}} \mathrm{a}^{3}$ & $\mathrm{p}^{\mathrm{h}} \mathrm{a}^{3}$ & $\mathrm{pfa}^{3}$ & $\mathrm{fa}^{3}$ & $\mathrm{fa}^{3}$ & $\mathrm{p}^{\mathrm{h}} \mathrm{a}^{3}$ \\
\hline 還 & 'to return' & $\mathrm{p}^{\mathrm{h}} \mathrm{e}^{4}$ & $\mathrm{p}^{\mathrm{h}} \mathrm{J}^{4}$ & $\mathrm{pf} \mathrm{J}^{4}$ & $\mathrm{fo}^{4}$ & $f 3^{4}$ & $\mathrm{p}^{\mathrm{h}} \mathrm{J}^{4}$ \\
\hline 雨 & 'rain' & $\mathrm{p}^{\mathrm{h}} \mathrm{an}^{1}$ & $\mathrm{p}^{\mathrm{h}} \mathrm{n}^{1}$ & pfen $^{1}$ & fun ${ }^{1}$ & fun 1 & $\mathrm{p}^{\mathrm{h}} \mathrm{un}^{1}$ \\
\hline
\end{tabular}

9 CL stands for Changliu, YX for Yongxing, LT for Longtang, LSG for Linshigang, HT for Huangtong, and XY for Xinying. 


\begin{tabular}{|c|c|c|c|c|c|c|c|}
\hline Chinese & English & CL & $\mathbf{Y X}$ & LT & LSG & нт & $\mathbf{X Y}$ \\
\hline & & $\mathrm{p}^{\mathrm{h}}$ & $\mathrm{p}^{\mathrm{h}}$ & pf- & f- & f- & $\mathrm{p}^{\mathrm{h}_{-}}$ \\
\hline 扇子 & 'fan' & $\mathrm{p}^{\mathrm{h}} \mathrm{aj}^{2}$ & $\mathrm{p}^{\mathrm{h}} \mathrm{j}^{2}$ & $p f j j^{2}$ & $f ə j^{2}$ & $f \jmath j^{2}$ & $\mathrm{p}^{\mathrm{h}} \mathrm{j}^{2}$ \\
\hline 掏 & 'to take out' & $\mathrm{p}^{\mathrm{h}} \mathrm{o} \mathrm{P}^{8}$ & $\mathrm{p}^{\mathrm{h}_{\mathcal{E}} \mathrm{P}^{8}}$ & - & - & - & $\mathrm{p}^{\mathrm{h}} \partial \mathrm{P}^{8}$ \\
\hline
\end{tabular}

b Hansell (1988: 247) suggested that the word for 'sky' in Ong-Be could correspond to the word for 'cloud' in Tai.

Table 15 illustrates words that begin with an implosive $d$-. Because all the modern reflexes have $d$ - as the initial, ${ }^{*} d$ - is reconstruct for this corresponding set.

TABLE $15 \quad * d-$

\begin{tabular}{|c|c|c|c|c|c|c|c|}
\hline Chinese & English & CL & $\mathbf{Y X}$ & LT & LSG & нт & $\mathbf{X Y}$ \\
\hline & & d- & d- & d- & d- & d- & d- \\
\hline 眼 & 'eye' & $d a^{1}$ & $d a^{1}$ & $d a^{1}$ & $d a^{1}$ & $d a^{1}$ & $d a^{1}$ \\
\hline 石 & 'stone' & $\operatorname{din}^{2}$ & $\operatorname{din}^{2}$ & $\operatorname{din}^{2}$ & $\operatorname{din}^{2}$ & $\operatorname{din}^{2}$ & $\mathrm{din}^{2}$ \\
\hline 樹 & 'tree' & don ${ }^{3}$ & dən ${ }^{3}$ & don ${ }^{3}$ & dun $^{3}$ & dun ${ }^{3}$ & dun ${ }^{3}$ \\
\hline 陸龜 & 'turtle' & - & - & dכ $w^{3}$ & dכ $w^{3}$ & dכw $w^{3}$ & dəw $w^{3}$ \\
\hline 黃蜂 & ‘wasp' & $\mathrm{daw}^{4}$ & $\mathrm{daw}^{4}$ & daw ${ }^{4}$ & $\mathrm{daw}^{4}$ & daw $^{4}$ & daw 4 \\
\hline 祐 & 'to sun-dry' & dak ${ }^{7}$ & $\mathrm{dak}^{7}$ & $\mathrm{dak}^{7}$ & $\mathrm{dak}^{7}$ & $\mathrm{dak}^{7}$ & $\mathrm{dak}^{7}$ \\
\hline 織布機 & 'loom' & $d \varepsilon \mathrm{k}^{8}$ & $d \varepsilon \mathrm{k}^{8}$ & $d \varepsilon \mathrm{k}^{8}$ & - & dək ${ }^{8}$ & dək ${ }^{8}$ \\
\hline
\end{tabular}

As all varieties in Table 16 have the same initial segment $t$-, it is straightforward to reconstruct * $t$ for this proto-phoneme.

\begin{tabular}{|c|c|c|c|c|c|c|c|}
\hline Chinese & English & CL & $\mathbf{Y X}$ & $\mathbf{L T}$ & LSG & HT & $\mathbf{X Y}$ \\
\hline & & t- & t- & $t-$ & $\mathrm{t}-$ & $\mathrm{t}-$ & $\mathrm{t}-$ \\
\hline 背 (v.) & 'to carry' (on back) & $\mathrm{ta}^{4}$ & $\mathrm{ta}^{4}$ & $\mathrm{ta}^{4} ; \mathrm{ta}^{\mathrm{l}}$ & $\mathrm{ta}^{4}$ & $\mathrm{ta}^{4}$ & - \\
\hline 鳴叫 & 'to crow' & $\tan ^{1}$ & $\tan ^{1}$ & $\tan ^{1}$ & $\tan ^{1}$ & $\tan ^{1}$ & $\tan ^{1}$ \\
\hline 魚鉤 & 'fish hook' & $\operatorname{tin}^{3}$ & $\operatorname{tin}^{3}$ & $\operatorname{tin}^{3}$ & $\operatorname{tin}^{3}$ & $\operatorname{tin}^{3}$ & $\operatorname{tin}^{3}$ \\
\hline 茅草 & 'thatch grass' & $\mathrm{tia}^{2}$ & $\operatorname{tia}^{2}$ & $\mathrm{tia}^{2}$ & $\operatorname{tia}^{2}$ & $\mathrm{tia}^{2}$ & $\mathrm{tia}^{2}$ \\
\hline 水牛 & 'water buffalo' & tej ${ }^{3}$ & $\mathrm{t} \varepsilon j^{3}$ & tej $^{3}$ & təj $j^{3}$ & $t_{\partial j} j^{3}$ & təj $j^{3}$ \\
\hline 簑衣 & 'straw rain cape' & $\mathrm{t} \varepsilon j^{4}$ & $\mathrm{t} \varepsilon j^{4}$ & $\operatorname{tej}^{4}$ & təj ${ }^{4}$ & təj ${ }^{4}$ & təj ${ }^{4}$ \\
\hline 尾巴 & 'tail' & tu $P^{7}$ & tu? ${ }^{7}$ & tur? & $\operatorname{tu}^{8}-\mathrm{t}$ & tu? ${ }^{7}$ & tu? ${ }^{7}$ \\
\hline 洗(手) & 'to wash (hands)' & tuk $^{7}$ & tuk $^{7}$ & tuk $^{7}$ & $\operatorname{tuk}^{7}$ & $\operatorname{tuk}^{7}$ & $\operatorname{tuk}^{7}$ \\
\hline
\end{tabular}


All words in Table 17 begin with $k$ - Consequently, I reconstruct * $k$ - for this initial. Although ' $3 S^{\text {SG }}$ in Changliu shows a tonal irregularity, it is not uncommon in tonal languages for function words and numerals to have unexpected tonal behaviors compared to content words. ${ }^{10}$

TABLE $17 \quad *$

\begin{tabular}{|c|c|c|c|c|c|c|c|}
\hline Chinese & English & CL & $\mathbf{Y X}$ & $\mathbf{L T}$ & LSG & HT & $\mathbf{X Y}$ \\
\hline & & k- & k- & k- & k- & k- & k- \\
\hline 殺 & 'to kill' & $\mathrm{ka}^{3}$ & $\mathrm{ka}^{3}$ & $\mathrm{ka}^{3}$ & $\mathrm{ka}^{3}$ & $\mathrm{ka}^{3}$ & $\mathrm{ka}^{3}$ \\
\hline 他 & '3 SG' & $\begin{array}{l}\mathrm{k} \partial^{4}-\mathrm{t} ; \\
\mathrm{ku} \mathrm{u}^{4}(\mathrm{XIN})\end{array}$ & $\mathrm{k}^{2}$ & $\mathrm{ke}^{2}$ & $\mathrm{k} \partial^{2}$ & $\mathrm{k}^{2}$ & $\mathrm{k}^{2}$ \\
\hline 雞 & ‘chicken’ & $\mathrm{kaj}^{1}$ & $\mathrm{k} \varepsilon j^{1}$ & $\mathrm{kej}^{1}$ & $\mathrm{kaj}^{1}$ & $\mathrm{kaj}^{1}$ & $\mathrm{kaj}^{1}$ \\
\hline 屎 & 'excrement' & $\mathrm{kaj}^{4}$ & $\mathrm{kaj}^{4}$ & $\mathrm{kaj}^{4}$ & $\mathrm{kaj}^{4}$ & $\mathrm{kaj}^{4}$ & $\mathrm{kaj}^{4}$ \\
\hline 吃 & 'to eat' & kon ${ }^{1}$ & $\mathrm{kon}^{1}$ & $\mathrm{kon}^{1}$ & $\mathrm{kon}^{1}$ & $\mathrm{kon}^{1}$ & kon 1 \\
\hline 苦 & 'bitter' & $\mathrm{kam}^{2}$ & $\mathrm{kam}^{2}$ & $\mathrm{kam}^{2}$ & $\mathrm{kam}^{2}$ & $\mathrm{kam}^{2}$ & $\mathrm{kam}^{2}$ \\
\hline 晚上 & 'night' & $\mathrm{kim}^{4}$ & kom $^{4}$ & kom $^{4}$ & - & $\mathrm{kom}^{4}$ & $\mathrm{kJm}^{4}$ \\
\hline 頭蟲 & 'head louse' & $\mathrm{kat}^{7}$ & $\mathrm{~kat}^{7}$ & kat $^{7}$ & kat $^{7}$ & $\mathrm{~kat}^{7}$ & $\mathrm{~kat}^{7}$ \\
\hline 綁 & 'to tie' & $\mathrm{kat}^{8}$ & $\mathrm{~kat}^{8}$ & $\mathrm{ket}^{8}$ & $\mathrm{~kat}^{8}$ & $\mathrm{~kat}^{8}$ & $\mathrm{~kat}^{8}$ \\
\hline
\end{tabular}

After reconstructing * $\mathrm{k}$ - for Table 17 , we encounter another correspondence that is also [+velar], as presented in Table 18. Considering that $k^{h_{-}}>x$ - is a widely attested natural sound change, I reconstruct ${ }^{*} \mathrm{k}^{\mathrm{h}_{-}}$ for this correspondence set. Note that there are only a small number correspondence sets with this proto-phoneme as the initial, unlike its stop counterpart * $k$-.

\begin{tabular}{|c|c|c|c|c|c|c|c|}
\hline Chinese & English & CL & $\mathbf{Y X}$ & $\mathbf{L T}$ & LSG & HT & $\mathbf{X Y}$ \\
\hline & & $\mathbf{k}^{\mathbf{h}_{-}}$ & $\mathbf{k}^{\mathbf{h}_{-}}$ & kx- & $x^{-}$ & $\mathbf{x}^{-}$ & $\mathbf{k}^{\mathbf{h}_{-}}$ \\
\hline 輕 & 'light (not heavy)' & $\mathrm{k}^{\mathrm{h}} \mathrm{e}^{3}$ & $\mathrm{k}^{\mathrm{h}} \mathrm{j}^{3}$ & $\mathrm{kx}^{3}$ & $\mathrm{xo}^{3}$ & $x 3^{3}$ & $\mathrm{k}^{\mathrm{h}} \mathrm{j}^{3}$ \\
\hline 重 & 'heavy' & $\mathrm{k}^{\mathrm{h}} \mathrm{en}^{1}$ & $\mathrm{k}^{\mathrm{h}} \mathrm{On}^{1}$ & kxวn $^{1}$ & xon $^{1}$ & xכn ${ }^{1}$ & $\mathrm{k}^{\mathrm{h}} \mathrm{\jmath n}^{1}$ \\
\hline 半夜 & 'midnight' & - & - & $-k x \partial n^{2}$ & -xən ${ }^{2}$ & $-\mathrm{x}^{2} \mathrm{n}^{2}$ & $-\mathrm{k}^{\mathrm{h}} \mathrm{n}^{2}$ \\
\hline 蟹 & 'crab' & $\mathrm{k}^{\mathrm{h}} \mathrm{Jm}^{2}$ & $\mathrm{k}^{\mathrm{h}} \mathrm{\jmath m}^{2}$ & $\mathrm{kx \supset m}^{2}$ & $\mathrm{xum}^{2}$ & $\mathrm{xum}^{2}$ & $\mathrm{k}^{\mathrm{h}} \mathrm{um}^{2}$ \\
\hline 癩蛤蟆 & 'toad' & $-\mathrm{k}^{\mathrm{h}} \mathrm{ok}^{7}$ & $-\mathrm{k}^{\mathrm{h}} \mathrm{ok}^{7}$ & $-\mathrm{kxok}^{7}$ & - & $-\mathrm{xok}^{7}$ & $-\operatorname{kxok}^{7}(\mathrm{HM})$ \\
\hline 貴 & 'expensive' & $\mathrm{k}^{\mathrm{h}} \mathrm{\jmath k}^{8}$ & $\mathrm{k}^{\mathrm{h}} \mathrm{\jmath k}^{8}$ & kxok $^{8}$ & $\mathrm{xok}^{8}$ & $\mathrm{x} \mathrm{k}^{8}$ & $\mathrm{k}^{\mathrm{h}} \mathrm{Jk}^{8}$ \\
\hline
\end{tabular}

10 For instance, the negator in Mandarin, 不 (bù), is pronounced with a falling tone. But when it is used to negate a verb that carries a falling tone, the tone of this negator has to change to a rising one. The numeral one, - (yī), in Mandarin has a level tone in its citation form. When followed by a classifier, it can be pronounced with a rising tone or a falling tone, depending on the tone of the classifier that follows. 
The words given in Table 19 all begin with a glottal stop; hence * 2 - is reconstructed for this set.

TABLE 19 *?

\begin{tabular}{|c|c|c|c|c|c|c|c|}
\hline Chinese & English & CL & $\mathbf{Y X}$ & LT & LSG & HT & $\mathbf{X Y}$ \\
\hline & & ?- & ?- & ?- & ?- & ?- & ?- \\
\hline 咳嗽 & 'to cough' & $\mathrm{Paj}^{1}$ & Paj $^{1}$ & $\mathrm{Pej}^{{ }^{1}-}$ & Paj $^{1}$ & Paj $^{1}$ & $-\mathrm{Paj}^{1}$ \\
\hline 拿 & 'to take' & Pow $^{1}$ & Pow ${ }^{1}$ & $30^{1}$ & Pow ${ }^{1}$ & $30 \mathrm{w}^{1}$ & - \\
\hline 甜、美味 & 'sweet; delicious' & $\operatorname{Pen}^{3}$ & Pian $^{3}$ & $\mathrm{Pen}^{3}$ & $? \varepsilon n^{3}$ & $? \varepsilon n^{3}$ & $? \varepsilon n^{3}$ \\
\hline 賣 ～～～～～～ & 'to sell' & $2 i \eta^{3}$ & $2 i \eta^{3}$ & $1 \varepsilon \eta^{3}$ & $\mathrm{Pi \eta}^{3}$ & $\mathrm{in}^{3}$ & $\operatorname{Pin}^{3}$ \\
\hline 粽子 & 'zongzi' & $\operatorname{Pot}^{7}$ & $\operatorname{Pot}^{7}$ & $\operatorname{Pot}^{7}$ & $\operatorname{Pot}^{7}$ & $\operatorname{Pot}^{7}$ & $\operatorname{Pot}^{7}$ \\
\hline 胸膛 & 'chest' & - Puak $^{7}$ & $-30 \mathrm{k}^{7}$ & $-30 \mathrm{k}^{7}$ & $-3 \partial \mathrm{k}^{7}$ & $-3 \partial \mathrm{k}^{7}$ & $-3 \partial \mathrm{k}^{7}$ \\
\hline 烏鴉 & 'crow' & $-\mathrm{Pak}^{8}$ & $-\mathrm{Pak}^{8}$ & $-\mathrm{Pak}^{8}$ & $-\mathrm{Pak}^{8}$ & $-\mathrm{Pak}^{8}$ & $-\mathrm{Pak}^{8}$ \\
\hline
\end{tabular}

\subsubsection{Fricatives}

I reconstruct Proto-Ong-Be *v for the correspondence set in Table 20. Considering the majority-wins principle, $v$ - is the favored candidate. It is noteworthy that ${ }^{*} 6$ - and *v- have merged in Changliu. In Yongxing and Longtang, *v- has split into $b$ - and $v$-, with $b$ - conditioned by following *-i- (Chen 2018).

\begin{tabular}{|c|c|c|c|c|c|c|c|}
\hline Chinese & English & CL & $\mathbf{Y X}$ & LT & LSG & HT & $\mathrm{XY}$ \\
\hline & & $6-$ & v-; 6- & $\mathbf{v - ; 6 -}$ & $v-$ & v- & v- \\
\hline 大腿 & 'thigh' & $-6 a^{2}$ & $-\mathrm{va}^{2}$ & $-\mathrm{va}^{2}$ & $-\mathrm{va}^{2}$ & $-\mathrm{va}^{2}$ & $-\mathrm{va}^{2}$ \\
\hline 村 & 'village' & $6 e^{3}$ & vo ${ }^{3}$ & vo ${ }^{3}$ & $\mathrm{vo}^{3}$ & $\mathrm{v} o^{3}$ & $v o^{3}$ \\
\hline 細糠 & 'rice bran' & $6 e^{4}$ & $\mathrm{v} \supset^{4}$ & $v o^{4}$ & $v^{4}$ & $v \supset^{4}$ & $v \jmath^{4}$ \\
\hline 飛 & 'to fly' & $\operatorname{ban}^{1}$ & $6 n^{1}$ & $6 e n^{1}$ & $\operatorname{vin}^{1}$ & $\operatorname{vin}^{1}$ & $\operatorname{vin}^{1}$ \\
\hline 太陽、白天 & 'sun; day' & $6 a n^{2}$ & vวn $^{2}$ & von $^{2}$ & vən $^{2}$ & vən $^{2}$ & vən $^{2}$ \\
\hline 風 & 'wind' & $6 a^{3}$ & $\operatorname{van}^{3}$ & $\operatorname{van}^{3}$ & $\operatorname{van}^{3}$ & $\operatorname{van}^{3}$ & $\operatorname{van}^{3}$ \\
\hline 蒼蠅 & 'fly' & $-\mathrm{ma \eta}^{4}-\mathrm{i}$ & $-\mathrm{van}^{4}$ & $-v^{4}{ }^{4}$ & $-\mathrm{va \eta}^{4}$ & $-\mathrm{va \eta}^{4}$ & $-\mathrm{va \eta}^{4}$ \\
\hline 薄 & 'thin (not thick)' & bian $^{1}$ & $\operatorname{biay}^{1}$ & biay $^{1}$ & viaj $^{1}$ & viaj $^{1}$ & viay $^{1}$ \\
\hline 買 & 'to buy' & $\operatorname{bian}^{1}$ & bien ${ }^{1}$ & $6 \varepsilon n^{1}$ & vi६n ${ }^{1}$ & $\operatorname{vian}^{1}$ & $\operatorname{vian}^{1}$ \\
\hline 柴 & 'firewood' & $6 ə n^{2}$ & vən $^{2}$ & vən $^{2}$ & vən $^{2}$ & vən $^{2}$ & vən $^{2}$ \\
\hline 旱地 & 'dry field’ & - & - & $6 \mathrm{in}^{3}$ & vən $^{3}$ & vən $^{3}$ & vən $^{3}$ \\
\hline 肩膀 & 'shoulder' & $-6 i^{3}$ & $-6 i^{3}$ & $-6 i^{3}$ & $-\mathrm{via}^{3}$ & $-v^{3}{ }^{3}$ & -via ${ }^{3}$ arm \\
\hline 火 & 'fire' & $6 \varepsilon j^{2}$ & $v \varepsilon j^{2}$ & $v_{e j}^{2}$ & vəj ${ }^{2}$ & vəj $^{2}$ & vəj $^{2}$ \\
\hline 吹(風) & 'to blow' & bew 4 & $v^{2} w^{4}$ & $v^{2} w^{4}$ & $v^{4} w^{4}$ & vวw $^{4}$ & vow $^{4}$ \\
\hline
\end{tabular}


${ }^{*}$ s- is selected for Table 21 because all the modern reflexes are realized as $s$-. Note that, while the correspondence set in Ong-Be varieties surveyed in this paper have $s^{-}$, the corresponding segment in Jizhao Haihua is $t s$-, as in $t$ siom $^{31}$ 'skinny', $t$ siai ${ }^{31}$ 'moon', and $t s^{\prime} y^{33}$ 'chopsticks' (Ostapirat 1998).

TABLE $21 \quad{ }^{*} S$ -

\begin{tabular}{|c|c|c|c|c|c|c|c|}
\hline Chinese & English & CL & $\mathbf{Y X}$ & $\mathbf{L T}$ & LSG & HT & $\mathbf{X Y}$ \\
\hline & & s- & s- & s- & s- & s- & s- \\
\hline 月亮 & 'moon' & $\mathrm{saj}^{1}$ & saj $^{1}$ & $\operatorname{sej}^{1}$ & saj $^{1}$ & saj $^{1}$ & saj $^{1}$ \\
\hline 耳朵 & ‘ear’ & $\mathrm{sa}^{1}$ & $\mathrm{sa}^{1}$ & $\mathrm{sa}^{1}$ & $\mathrm{sa}^{1}$ & $\mathrm{sa}^{1}$ & $\mathrm{sa}^{1}$ \\
\hline 筷子 & 'chopsticks' & $\mathrm{so}^{4}$ & səw $^{4}$ & $s \partial^{4}$ & səw $^{4}$ & səw $^{4}$ & səw $^{4}$ \\
\hline 瘦 & 'skinny' & $\operatorname{sum}^{1}$ & $\operatorname{sum}^{1}$ & $\operatorname{sum}^{1}$ & $\operatorname{sum}^{1}$ & $\operatorname{sum}^{1}$ & $\operatorname{sum}^{1}$ \\
\hline 蜜蜂 & 'bee' & $\operatorname{sa\eta }^{3}$ & $\operatorname{sa\eta }^{3}$ & $\operatorname{sa\eta }^{3}$ & $\operatorname{sa\eta }^{3}$ & $\operatorname{sa\eta }^{3}$ & $\operatorname{sa\eta }^{3}$ \\
\hline 漁網 & 'fishnet' & $\mathrm{saj}^{3}$ & saj $^{3}$ & saj $^{3}$ & $\mathrm{saj}^{3}$ & $\mathrm{saj}^{3}$ & $\mathrm{saj}^{3}$ \\
\hline 䟽菜 & 'vegetable' & $\operatorname{sak}^{7}$ & $\operatorname{sak}^{7}$ & $\operatorname{sak}^{7}$ & $\operatorname{sak}^{7}$ & $\operatorname{sak}^{7}$ & $\operatorname{sak}^{7}$ \\
\hline 寺頭 & 'taro' & $\mathrm{sak}^{7}$ & $\mathrm{sak}^{7}$ & $-\mathrm{sak}^{7}$ & $-\mathrm{sak}^{7}$ & $-\mathrm{sak}^{7}$ & $-\mathrm{sak}^{7}$ \\
\hline
\end{tabular}

Two reflexes z- and $z$ - are observed in Table 22. Considering the place of articulation, where one is alveolar and the other palatal, I propose that ${ }^{*} z$ - should be reconstructed as the initial for this sound correspondence $(z: z: z: z: z: z)$, because ${ }^{*} \mathrm{z}$ - is better reserved for the corresponding sets in Table 23 where a majority of reflexes are alveolar (z:z:z:z:z l:l:l).

TABLE $22 \quad{ }^{*} z_{-}$

\begin{tabular}{|c|c|c|c|c|c|c|c|}
\hline Chinese & English & CL & $\mathbf{Y X}$ & $\mathbf{L T}$ & LSG & HT & $\mathbf{X Y}$ \\
\hline & & $\mathbf{z}^{-}$ & $\mathbf{z}^{-}$ & $\mathbf{z}^{-}$ & Z- & Z- & $\mathrm{Z}^{-}$ \\
\hline 老虎 & 'tiger' & $\mathrm{zua}^{1}$ & $\mathrm{zua}^{1}$ & zua $^{1}$ & zua $^{1}$ & $\begin{array}{l}\text { zua' } \\
\text { 'lion' (LC) }\end{array}$ & - \\
\hline 藥 & 'medicine' & $\mathrm{zia}^{1}$ & $\mathrm{zia}^{1}$ & $\mathrm{zia}^{1}$ & $\mathrm{zia}^{1}$ & $\operatorname{zia}^{1}$ & zia $^{1}$ \\
\hline 椰子 & 'coconut' & $-\mathrm{zia}^{2}$ & $-\mathrm{zia}^{2}$ & $-\mathrm{zia}^{2}$ & $-\mathrm{zia}^{2}$ & $-\mathrm{zia}^{2}$ & $-\mathrm{zia}^{2}$ \\
\hline 木瓜 & 'papaya' & $\mathrm{zia}^{2}-$ & - & $\mathrm{kia}^{2}-;$ pia $^{2}-$ & $-\mathrm{zia}^{2}-$ & $\mathrm{zial}^{2}$ & $\mathrm{zia}^{2}-$ \\
\hline 酒 & 'liquor' & $\operatorname{zan}^{3}$ & $\operatorname{zan}^{3}$ & $\operatorname{zan}^{3}$ & $\operatorname{zan}^{3}$ & $\operatorname{zan}^{3}$ & $\operatorname{zan}^{3}$ \\
\hline 油 & 'oil' & $\mathrm{zJw}^{2}$ & ${\mathrm{z} \partial \mathrm{w}^{2}}^{2}$ & ${\mathrm{z} \partial \mathrm{w}^{2}}^{2}$ & zəow $^{2}$ & $\mathrm{zu}^{2}$ & $\mathrm{zu}^{2}$ \\
\hline 衣服 & 'clothes' & $-z^{3} a^{3}$ & $\mathrm{zua}^{3}$ & $-z^{3} a^{3}$ & zua $^{3}$ & - zua $^{3}$ & - zua $^{3}$ \\
\hline 餓 & 'hungry' & $\mathrm{zak}^{7}$ & $\mathrm{zak}^{7}$ & $\mathrm{zak}^{7}$ & ziak $^{7}$ & ziak $^{7}$ & ziak $^{7}$ \\
\hline
\end{tabular}


Words listed in Table 23 have $z$-, $z$ - or $l$ - as reflexes. Because I have already reconstructed ${ }^{*} z$ - for Table 22 , and because *s- has been taken by Table 21, another proto phoneme must be selected as the placeholder. Given that most reflexes are alveolar, I reconstruct ${ }^{*} \mathrm{z}$ - for this table.

\begin{tabular}{|c|c|c|c|c|c|c|c|c|}
\hline Chinese & English & CL & $\mathbf{Y X}$ & $\mathbf{L T}$ & LSG & HT & JL & $\mathbf{X Y}$ \\
\hline & & $\mathrm{z}^{-}$ & $\mathrm{z}^{-}$ & z- & Z- & z- or l- & l- & l- \\
\hline 裡面 & 'inside' & $\mathrm{ze}^{1}$ & $\mathrm{zJ}^{1}$ & $\mathrm{z} J^{1}$ & $\mathrm{zo}^{1}$ & - & $\log$ & $\mathrm{l}^{1}(\mathrm{HM})$ \\
\hline 林投果 & 'pandanus fruit' & $-\mathrm{za}^{4}$ & $-\mathrm{za}^{4}$ & $-\mathrm{za}^{4}$ & $-\mathrm{za}^{4}$ & $\mathrm{la}^{4}$ & $-\mathrm{la}^{4}$ & $-\mathrm{la}^{4}$ \\
\hline 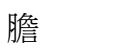 & 'gall' & $z^{3} j^{1}$ & $\mathrm{zaj}^{1}$ & $\mathrm{zej}^{1}$ & $\mathrm{zoj}^{1}$ & $z^{2} j^{1}$ & $\operatorname{loj}^{1}$ & $\operatorname{loj}{ }^{1}$ \\
\hline 黑色 & 'black' & $\mathrm{zam}^{1}$ & $\mathrm{zam}^{1}$ & zem $^{1}$ & $\mathrm{zam}^{1}$ & $\operatorname{zam}^{1}$ & $\operatorname{lam}^{1}$ & $\operatorname{lam}^{1}$ \\
\hline 兔子 & 'rabbit' & $\operatorname{zunn}^{3}$ & $\operatorname{zin}^{3}$ & $\operatorname{zin}^{3}$ & $\operatorname{zon}^{3}$ & $\operatorname{lon} n^{3}$ & $\operatorname{lon}^{3}$ & $\operatorname{lən} n^{3}$ \\
\hline 房屋 & 'house' & $\operatorname{zan}^{2}$ & $\operatorname{zan}^{2}$ & $\operatorname{zan}^{2}$ & $\operatorname{zan}^{2}$ & $\operatorname{zan}^{2}$ & $\operatorname{lan}^{2}$ & $\operatorname{lan}^{2}$ \\
\hline 篩子 & 'winnowing basket' & $\mathrm{zJ \eta}^{2}$ & $\mathrm{zJ \eta}^{2}$ & $\mathrm{zon}^{2}$ & $\mathrm{zOJ}^{2}$ & $\operatorname{lo\eta } \eta^{2}$ & $\operatorname{lo\eta } \eta^{2}$ & $\operatorname{lo\eta } \eta^{2}$ \\
\hline 鹹 & 'salty' & $\operatorname{za\eta j}^{3}$ & $\operatorname{zan}^{3}$ & $\operatorname{zan}^{3}$ & $\operatorname{zan}^{3}$ & $\operatorname{la\eta }^{3}$ & $\operatorname{lan}^{3}$ & $\operatorname{la\eta }^{3}$ \\
\hline 生 (肉) & 'raw' & $\mathrm{zop}^{7}$ & $z^{\prime} p^{7}$ & zop $^{7}$ & zəp $^{7}$ & $\operatorname{zip}^{7}$ & $\operatorname{lip}^{7}$ & $\operatorname{lip}^{7}$ \\
\hline 米 & 'husked rice' & $\operatorname{zap}^{8}$ & zap $^{8}$ & zep ${ }^{8}$ & zop 8 & zop 8 & $\operatorname{lop}^{8}$ & lop 8 \\
\hline 蜈蚣 & 'centipede’ & zJp $^{8}$ & $-z \supset p^{8}$ & -zep ${ }^{8}$ & zәр 8 & $\operatorname{zip}^{8}$ & $\operatorname{lip}^{8}$ & $\operatorname{lip}^{8}$ \\
\hline
\end{tabular}

It is noteworthy that Huangtong and Maniao have two variants, $z$ - and $l-$, as their reflexes of * $\mathrm{z}-$. $z$ - and $l$ - are free variants in Huangtong and Maniao, and inter- and intraspeaker variation is observed, as exemplified in Table 24. I propose that this is due to their proximity to languages with different reflexes. To the east of Huangtong lie the varieties that reflect ${ }^{*} z^{-}$as $z^{-}$, and to the west of Huangtong are the varieties that have $l$ - for ${ }^{*} \mathrm{z}$-. As a result of language contact, Huangtong employs two free variants for the same proto initial. The Jialai $(\mathrm{JL})$ data are included in Table 23 as a basis for comparison.

TABLE $24 \quad{ }^{11}$ Free variants in Huangtong and Maniao

\begin{tabular}{|c|c|c|c|c|c|}
\hline Chinese & English & MN (Xin 2007) & MN (mine) & HT (Xin 2007) & HT (mine) \\
\hline 房屋 & 'house' & $\operatorname{zan}^{2}$ & $\operatorname{lan}^{2}$ & $\operatorname{zan}^{2}$ & $\operatorname{zan}^{2}$ \\
\hline 㙐子 & 'mat' & ziu $^{4}$ & ziw $^{4}$ & ziu $^{4}$ & $\operatorname{liw}^{4}$ \\
\hline 黑 & 'black' & $\operatorname{zam}^{1}$ & $\operatorname{lam}^{1}$ & $\operatorname{zam}^{1}$ & $\operatorname{zam}^{1} \sim \operatorname{lam}^{1}$ \\
\hline 鼻 & 'nose' & Zoग ${ }^{1}$ & $\operatorname{lo\eta }{ }^{1} \sim \mathrm{z}^{1}$ & Zכ\'1 & $\operatorname{lo\eta }^{1}$ \\
\hline 米 & 'husked rice' & zop 8 & lэp 8 & zop 8 & zop 8 \\
\hline 指甲 & 'nail' & - & zip 8 & - & $\operatorname{lip}^{8}$ \\
\hline
\end{tabular}

All the words in Table 25 begin with $h$-. For this reason, *h- is reconstructed as the proto initial.

11 The coda in 'mat' is analyzed as a vowel in Xin (2007), but as a glide in my system. 
TABLE $25 * h$ -

\begin{tabular}{|c|c|c|c|c|c|c|c|}
\hline Chinese & English & CL & $\mathbf{Y X}$ & $\mathbf{L T}$ & LSG & HT & $\mathbf{X Y}$ \\
\hline & & h- & h- & h- & h- & h- & h- \\
\hline 梳子 & 'comb' & haj $^{1}$ & haj $^{1}$ & hej $^{1}$ & haj $^{1}$ & haj $^{1}$ & haj $^{1}$ \\
\hline 含 & 'to hold in mouth' & $\mathrm{ham}^{2}$ & ham $^{2}$ & (h) $\mathrm{am}^{2}$ & $\mathrm{ham}^{2}$ & həm $^{2}$ & hom $^{2}$ \\
\hline 者(飯) & 'to cook (rice)' & hoy $^{2}$ & hū ${ }^{2}$ & hoy $^{2}$ & huj ${ }^{2}$ & hup $^{2}$ & huy $^{2}$ \\
\hline 柱子 & 'pillar' & - & həw ${ }^{1}$ & hew ${ }^{1}$ & how $^{1}$ & hวw ${ }^{1}$ & həw $^{1}$ \\
\hline 穿山甲 & 'pangolin' & $\operatorname{han}^{4}$ & hon 4 & hวn 4 & hən 4 & $\operatorname{hin}^{4}$ & $\operatorname{hin}^{4}$ \\
\hline 酸 & 'sour' & hua $^{3}$ & hua $^{3}$ & hua $^{3}$ & hua $^{3}$ & $(\mathrm{~h}) \mathrm{ua}^{3}$ & hua $^{3}$ \\
\hline 挑(擔) & $\begin{array}{l}\text { 'to carry (a pole) on the } \\
\text { shoulder' }\end{array}$ & $\operatorname{hap}^{7}$ & hap $^{7}$ & hap $^{7}$ & hap $^{7}$ & hap $^{7}$ & hap $^{7}$ \\
\hline 布 & 'cloth' & hap $^{8}$ & hap $^{8}$ & hep ${ }^{8}$ & həp 8 & həp 8 & həp 8 \\
\hline
\end{tabular}

\subsubsection{The Proto-affricate *ts-}

Only a handful of words in Ong-Be begin with /ts/, which can be realized as [ts] or [ts $\left.{ }^{\mathrm{h}}\right]$ in all varieties surveyed. That is, aspiration is not phonemic for this affricate. Since all of these words have an identical affricate initial, *ts- is reconstructed for Table 26. Many of them are early Chinese loans, such as 灶 'kitchen range; hearth' (Middle Chinese:12 tsa u), 滴 ‘(to) drop' (Middle Chinese: tiek), 鹿 'deer' (Middle Chinese: luk), and 藍 'blue; indigo' (Middle Chinese: lam). But based on the internal evidence, these etyma show a regular correspondence as those of the native stratum, indicating that they are early loans borrowed before the branch-off of Proto-Ong-Be.

TABLE $26 *$ *t-

\begin{tabular}{|c|c|c|c|c|c|c|c|}
\hline Chinese & English & CL & $\mathbf{Y X}$ & $\mathbf{L T}$ & LSG & HT & $\mathbf{X Y}$ \\
\hline & & $t s \sim t s^{h}$ & ts $\sim t^{h}$ & ts $\sim t^{h}$ & ts $\sim t^{h}$ & $t s \sim t s^{h}$ & ts $\sim t^{h}$ \\
\hline 小鈸 & 'a type of cymbals' & - & $-\mathrm{ts}^{\mathrm{h}} \mathcal{E}^{1}$ & $-\mathrm{ts}^{\mathrm{h}} \mathcal{E}^{1}$ & - & $\mathrm{ts}^{\mathrm{h}} \mathcal{E}^{1}$ & $\mathrm{ts}^{\mathrm{h}} \mathcal{E}^{1}$ \\
\hline 腸 & 'intestine' & - & - & $-\mathrm{ts}^{\mathrm{h}} \mathrm{e}^{4}$ & - & $\mathrm{ts}^{\mathrm{h}} \varepsilon^{4}$ & $\mathrm{ts}^{\mathrm{h}} \varepsilon^{4}$ \\
\hline 下午 & 'afternoon' & - tsew $^{2}$ & $-\mathrm{ts}^{\mathrm{h}} \mathrm{\partial w^{2 }}$ & - & $-\mathrm{tsow}^{2}$ & $-\mathrm{ts}^{\mathrm{h}} \mathrm{\partial \textrm {w } ^ { 2 }}$ & $-\mathrm{ts}^{\mathrm{h}} \mathrm{\partial w^{2 }}$ \\
\hline 灶 & 'kitchen range' & ts ${ }^{h} \mathrm{aw}^{3}{ }_{-}$ & $\mathrm{ts}^{\mathrm{h}} \mathrm{aw}^{3}$ & tsaw $^{3}$ & tsaw $^{3}$ & $\mathrm{ts}^{\mathrm{h}} \mathrm{aw}^{3}$ & $\mathrm{ts}^{\mathrm{h}} \mathrm{aw}^{3}$ \\
\hline 監靛草 & 'blue; indigo' & $\mathrm{ts}^{\mathrm{h}} \mathrm{am}^{2}$ & - & $\operatorname{tsam}^{2}(\mathrm{~L} \& \mathrm{z})$ & - & $\operatorname{tsam}^{2}(\mathrm{LC})$ & - \\
\hline 滴 (v.) & 'to drop' & $\mathrm{ts}^{\mathrm{h}} \mathrm{P}^{7}$ & tshi? ${ }^{7}$ & ts $^{h_{i} P^{7}}$ & $t s^{(h)} \partial P^{7}$ & $\mathrm{ts}^{\mathrm{h}} \partial \mathrm{P}^{7}$ & $\mathrm{ts}^{\mathrm{h}} \mathrm{P}^{7}$ \\
\hline 溼 & 'wet' & $\mathrm{ts}^{\mathrm{h}} \mathrm{ak}^{8}$ & tsak $^{8}$ & $\mathrm{ts}^{\mathrm{h}} \mathrm{ek}^{8}$ & tsak $^{8}$ & $\mathrm{ts}^{\mathrm{h}} \mathrm{ak}^{8}$ & $\mathrm{ts}^{\mathrm{h}} \mathrm{ak}^{8}$ \\
\hline 鹿 & 'deer’ & ts $^{\text {h }}$ uak $^{8}$ & $\mathrm{ts}^{\mathrm{h}} \mathrm{ok}^{8}$ & $\mathrm{ts}^{(\mathrm{h})} \mathrm{Ok}^{8}$ & $\mathrm{ts}^{\mathrm{h}} \mathrm{ok}^{8}$ & tsok $^{8}$ & ts $^{\mathrm{h}} \mathrm{ok}^{8}$ \\
\hline
\end{tabular}

12 Middle Chinese cited in this study is based on Wang Li's (王力) reconstruction from Corpus of Old Chinese, Middle Chinese and Modern Chinese (漢字古今音資料庫), edited by Department of Chinese Literature (National Taiwan University) \& Institute of Information Science (Academia Sinica), n.d. 


\subsubsection{Sonorants}

Table 27 illustrates words that begin with $l$-. Based on the internal evidence, ${ }^{*}$ - is straightforwardly reconstructed for this proto-initial.

TABLE $27 \quad *$ -

\begin{tabular}{|c|c|c|c|c|c|c|c|}
\hline Chinese & English & CL & $\mathbf{Y X}$ & LT & LSG & нт & $\mathbf{X Y}$ \\
\hline & & l- & l- & l- & $1-$ & l- & l- \\
\hline 秧苗 & 'rice seedling' & $\mathrm{la}^{3}$ & $\mathrm{la}^{3}$ & $\mathrm{la}^{3}$ & $\mathrm{la}^{3}$ & $l^{3}$ & $\mathrm{la}^{3}$ \\
\hline 藍 & 'blue' & $\operatorname{lam}^{2}$ & $\operatorname{lam}^{2}$ & $\operatorname{lam}^{2}$ & $\operatorname{lam}^{2}$ & $\operatorname{lam}^{2}$ & $\operatorname{lam}^{2}$ \\
\hline 黃色 & 'yellow' & $\operatorname{lay}^{1}$ & $\operatorname{lag}^{1}$ & $\operatorname{lag}^{1}$ & $\operatorname{lay}^{1}$ & $\operatorname{lag}^{1}$ & $\operatorname{lay}^{1}$ \\
\hline 舌頭 & 'tongue' & $\operatorname{lin}^{4}$ & $\operatorname{lin}^{4}$ & $\operatorname{lin}^{4}$ & $\operatorname{lin}^{4}$ & $\operatorname{lin}^{4}$ & $\operatorname{lin}^{4}$ \\
\hline 多 & 'many, much' & $\operatorname{liaw}^{4}$ & liaw $^{4}$ & $\operatorname{liaw}^{4}$ & liaw $^{4}$ & liaw $^{4}$ & liaw $^{4}$ \\
\hline 魚鱗 & 'fish scale' & $\operatorname{lum} P^{7}$ & $\operatorname{li} ?^{7}$ & $\operatorname{li} ?^{7}$ & $\operatorname{li} P^{8}-\mathrm{t}$ & $\operatorname{li}^{7}$ & $\operatorname{li}^{7}$ \\
\hline 睡 、躺 & 'to sleep; to lie down' & $\operatorname{lap}^{7}$ & $\operatorname{lap}^{7}$ & $\operatorname{lap}^{7}$ & $\operatorname{lap}^{7}$ & $\operatorname{lap}^{7}$ & $\operatorname{lap}^{7}$ \\
\hline 蟫筒闻 & 'cocoon of the silkworm' & $\operatorname{luk}^{7}$ & $\operatorname{luk}^{7}$ & $\operatorname{luk}^{7}$ & - & $\operatorname{luk}^{7}$ & - \\
\hline 深 & 'deep’ & $\operatorname{lak}^{8}$ & $\mathrm{lak}^{8}$ & $\operatorname{lek}^{8}$ & $\mathrm{lak}^{8}$ & $\mathrm{lak}^{8}$ & $\mathrm{lak}^{8}$ \\
\hline 兒女 & 'offspring' & $\mathrm{l}_{\varepsilon \mathrm{k}^{8}}$ & $\mathrm{l}_{\varepsilon \mathrm{k}^{8}}$ & $\mathrm{l}_{\varepsilon \mathrm{k}^{8}}$ & ${\mathrm{l} ə \mathrm{k}^{8}}$ & $l \mathrm{k}^{8}$ & $\mathrm{lək}^{8}$ \\
\hline
\end{tabular}

All the words in Table 28 begins with $m$-, so * $\mathrm{m}$ - is reconstructed as the proto-initial for these cognate sets.

TABLE $28 \quad * m$ -

\begin{tabular}{|c|c|c|c|c|c|c|c|}
\hline Chinese & English & CL & $\mathbf{Y X}$ & LT & LSG & HT & $\mathbf{X Y}$ \\
\hline & & m- & m- & m- & m- & m- & m- \\
\hline 客人 & 'guest' & $m e^{1}$ & $m \jmath^{1}$ & - & $\mathrm{mo}^{1}$ & $m \jmath^{1}$ & $m \jmath^{1}$ \\
\hline 手 & 'hand' & $\mathrm{me}^{2}$ & $\mathrm{~m} \mathrm{\jmath}^{2}$ & $m \jmath^{2}$ & $\mathrm{mo}^{2}$ & $m \jmath^{2}$ & $\mathrm{~m} \mathrm{\jmath}^{2}$ \\
\hline 甘蔗 & 'sugarcane' & $\mathrm{maj}^{3}$ & $\mathrm{maj}^{3}$ & $\mathrm{maj}^{3}$ & $\mathrm{maj}^{3}$ & maj $^{3}$ & $\mathrm{maj}^{3}$ \\
\hline 雌性 & 'female' & $\mathrm{maj}^{4}$ & $\mathrm{maj}^{4}$ & $\mathrm{maj}^{4}$ & $\mathrm{maj}^{4}$ & $\mathrm{maj}^{4}$ & $\mathrm{maj}^{4}$ \\
\hline 蟲 & 'bug; worm' & $\min ^{2}$ & $\min ^{2}$ & $m \varepsilon \eta^{2}$ & $\min ^{2}$ & $\min ^{2}$ & $\min ^{2}$ \\
\hline 稻草 & 'straw' & muy $^{4}$ & muy $^{4}$ & $\mathrm{mur}^{4}$ & muy $^{4}$ & muy $^{4}$ & muy $^{4}$ \\
\hline 跳蚤 & 'flea' & $\mathrm{mat}^{7}$ & mat $^{7}$ & mat $^{7}$ & mat $^{7}$ & $\mathrm{mat}^{7}$ & mat $^{7}$ \\
\hline 刀 & 'knife' & $\mathrm{mit}^{8}$ & $\mathrm{mit}^{8}$ & mit $^{8}$ & mit $^{8}$ & mit $^{8}$ & mit $^{8}$ \\
\hline 鼻涕 & 'mucus' & $\mathrm{muk}^{8}$ & juk $^{8}$ & $\mathrm{mok}^{8}$ & $\mathrm{muk}^{8}$ & $\mathrm{muk}^{8}$ & $\mathrm{muk}^{8}$ \\
\hline
\end{tabular}

The words in Table 29 begin with $n$ - in all varieties, so I reconstruct * $\mathrm{n}$-. 
TABLE $29 \quad * n-$

\begin{tabular}{|c|c|c|c|c|c|c|c|}
\hline Chinese & English & CL & $\mathbf{Y X}$ & LT & LSG & HT & $\mathbf{X Y}$ \\
\hline & & n- & n- & n- & n- & n- & n- \\
\hline 春(米) & 'to pound (rice)' & $\operatorname{nam}^{2}$ & $\operatorname{nam}^{2}$ & $\operatorname{nam}^{2}$ & $\operatorname{nam}^{2}$ & $\operatorname{nam}^{2}$ & $\operatorname{nam}^{2}$ \\
\hline 水 & 'water' & $\operatorname{nam}^{4}$ & $\operatorname{nam}^{4}$ & $\operatorname{nam}^{4}$ & $\operatorname{nam}^{4}$ & $\operatorname{nam}^{4}$ & $\operatorname{nam}^{4}$ \\
\hline 皮膚 & ‘skin’ & $\operatorname{nay}^{1}$ & $\operatorname{nay}^{1}$ & ney ${ }^{\prime}$ & $\operatorname{nay}^{1}$ & $\operatorname{na\eta }^{1}$ & nay ${ }^{1}$ \\
\hline 竹笛 & 'bamboo shoot' & $\operatorname{na\eta }^{2}$ & $\operatorname{na\eta }^{2}$ & $\operatorname{na\eta }^{2}$ & $\operatorname{nay}^{2}$ & $\operatorname{na\eta }^{2}$ & $\operatorname{na\eta }^{2}$ \\
\hline 厚 & 'thick' (not thin) & na $^{1}$ & $\mathrm{na}^{1}$ & $\mathrm{na}^{1}$ & $\mathrm{na}^{1}$ & na ${ }^{1}$ & na ${ }^{1}$ \\
\hline 臉 & 'face' & $n a^{3}$ & $\mathrm{na}^{3}$ & $\mathrm{na}^{3}$ & na ${ }^{3}$ & $\mathrm{na}^{3}$ & $\mathrm{na}^{3}$ \\
\hline 借 & 'to borrow; to lend' & naj $^{3}$ & naj $^{3}$ & naj $^{3}$ & naj $^{3}$ & naj $^{3}$ & naj $^{3}$ \\
\hline 水田 & 'paddy field' & nia $^{2}$ & $\operatorname{nia}^{2}$ & $\operatorname{nia}^{2}$ & nia $^{2}$ & $\operatorname{nia}^{2}$ & $\operatorname{nia}^{2}$ \\
\hline 冷 & 'cold' & nit $^{7}$ & nit $^{7}$ & nit $^{7}$ & nit $^{7}$ & nit $^{7}$ & nit $^{7}$ \\
\hline 鳥 & 'bird' & nuak $^{8}$ & $\operatorname{nok}^{8}$ & nok $^{8}$ & $\operatorname{nok}^{8}$ & nok $^{8}$ & $\operatorname{nok}^{8}$ \\
\hline
\end{tabular}

Table 30 illustrates words with a n:n:n:n:n:z sound correspondence, similar to the n:n:n:n:n:n correspondence in Table 29. Changliu has /na/ for both 'face' (in Table 29) and 'tree root', whereas Huangtong has /na/ for ' face' but $/ \mathrm{n} \mathrm{a/for} \mathrm{'tree} \mathrm{root'.} \mathrm{Considering} \mathrm{that} n$-and $n$ - can occur in the same environment in Huangtong, they must originate from different initials. Consequently, " $\mathrm{j}$ - is reconstructed for Table 30 . It is noteworthy that all varieties in Lingao County maintain the * $\mathrm{n}-{ }^{*} \mathrm{n}$ contrast, except for Xinying, in which * $\mathrm{n}$ - has lost nasality. By contrast, in Changliu, Yongxing, Longtang, and Linshigang, *n- has merged with *n-.

TABLE $30 \quad * n-$

\begin{tabular}{|c|c|c|c|c|c|c|c|}
\hline Chinese & English & CL & $\mathbf{Y X}$ & $\mathbf{L T}$ & LSG & HT & $\mathbf{X Y}$ \\
\hline & & n- & n- & n- & n- & n- & $z^{-}$ \\
\hline 樹根 & 'tree root' & $\mathrm{na}^{2}-$ & - & - & $\mathrm{na}^{2}-$ & $\mathrm{na}^{2}-$ & $\mathrm{za}^{2}-$ \\
\hline 玩 & 'to play'c & $\operatorname{nam}^{1}$ & $\operatorname{nam}^{33}-\mathrm{t}$ & - & $\operatorname{nam}^{1}$ & $\operatorname{jam}^{1}$ & $\operatorname{zam}^{1}$ \\
\hline 蛋 & ‘egg’ & nom ${ }^{1}$ & $\operatorname{nam}^{1}$ & nem ${ }^{1}$ & num $^{1}$ & num $^{1}$ & $\operatorname{zum}^{1}$ \\
\hline 蚊子 & 'mosquito' & - noy $^{2}$ & - nuy $^{2}$ & noy $^{2}$ & nur $^{2}$ & nur $^{2}$ & $\operatorname{zun}^{2}$ \\
\hline 監䀋 & 'salt' & naw $^{3}$ & naw $^{3}$ & naw $^{3}$ & naw $^{3}$ & naw $^{3}$ & $\mathrm{zaw}^{3}$ \\
\hline 韎 & 'pliable but strong' & nat $^{7}$ & nat $^{7}$ & nat $^{7}$ & nat $^{7}$ & jat $^{7}$ & zat $^{7}$ \\
\hline 縫(衣) & 'to sew' & - & $\operatorname{nap}^{7}-\mathrm{t}$ & nep $8^{\prime}(\mathrm{L} \& \mathrm{z})$ & nap $^{8}$ & nэp $^{8}(\mathrm{LC})$ & zכp 8 \\
\hline
\end{tabular}

c In Yongxing, the tonal realization for 'to play' is aberrant. The raised ${ }^{33}$ here refers to the pitch curve, not a tone category.

The correspondence set for * $y$ - is illustrated in Table 31 . While both Huangtong and Xinying have $\eta$ for 'to sit' and 'shadow', Changliu has $\eta$ - and $m$ - and Yongxing and Longtang have $m$ - instead. However, $m$ - seems to occur only before $-u$-. This appears to be a conditioned split, with * $y$ - assimilating to the labiality of the following vowel, a cross-linguistically common change. * $\mathrm{y}$ - is consequently reconstructed here. 


\begin{tabular}{|c|c|c|c|c|c|c|c|}
\hline \multirow[t]{2}{*}{ Chinese } & \multirow[t]{2}{*}{ English } & \multirow{2}{*}{$\begin{array}{l}\text { CL } \\
\text { y-, m-/_u }\end{array}$} & \multirow{2}{*}{$\begin{array}{l}\mathbf{Y X} \\
\mathrm{y-}, \mathbf{m}-/ / \mathbf{u}\end{array}$} & \multirow{2}{*}{$\begin{array}{l}\text { LT } \\
\text { y-, m-/_u }\end{array}$} & \multirow{2}{*}{$\frac{\text { LSG }}{\eta-}$} & \multirow{2}{*}{$\begin{array}{l}\text { HT } \\
\eta-\end{array}$} & \multirow{2}{*}{$\begin{array}{l}\mathbf{X Y} \\
\mathrm{y-}\end{array}$} \\
\hline & & & & & & & \\
\hline 坐 & 'to sit' & now $^{1}$ & $m u^{1}$ & $\mathrm{mu}^{1}$ & yәw ${ }^{1}$ & no ${ }^{1}$ & yo ${ }^{1}$ \\
\hline 影子 & 'shadow' & $\mathrm{muj}^{1}$ & muj ${ }^{1}$ & muj ${ }^{1}$ & juj $^{1}$ & juj $^{1}$ & puj $^{1}$ \\
\hline 蛇 & 'snake’ & nia $^{2}$ & jia $^{2}$ & yia $^{2}$ & nia $^{2}$ & nia $^{2}$ & nia $^{2}$ \\
\hline 乾飯 & 'cooked rice' & „aj $^{2}$ & gaj $^{2}$ & yaj $^{2}$ & yaj $^{2}$ & $\mathrm{yaj}^{2}$ & gaj $^{2}$ \\
\hline 哭 & 'to cry' & gaj $^{3}$ & naj $^{3}$ & naj $^{3}$ & "aj $^{3}$ & naj $^{3}$ & yaj $^{3}$ \\
\hline 熟 & 'ripe' & gaw $^{3}$ & jaw $^{3}$ & yaw $^{3}$ & yaw $^{3}$ & yaw $^{3}$ & yaw $^{3}$ \\
\hline 稻 & 'rice in the field' & yaw $^{4}$ & yaw $^{4}$ & yaw $^{4}$ & yaw $^{4}$ & yaw $^{4}$ & yaw $^{4}$ \\
\hline 啞 & 'mute' & nop 7 & рэр ${ }^{7}$ & рэр ${ }^{7}$ & nop 7 & yop $^{7}$ & nop 7 \\
\hline 柚子 & 'pomelo' & -1juak 8 & - jok $^{8}$ & - jok $^{8}$ & - jok $^{8}$ & $-\operatorname{jok}^{8}(\mathrm{MN})$ & - jok $^{8}$ \\
\hline 針 & 'needle' & yaP $^{8}$ & gar 8 & ya? & ya? $^{8}$ & yaP $^{8}$ & ya? 8 \\
\hline
\end{tabular}

\subsection{Finals}

This section deals with finals in Proto-Ong-Be. There are three nasal, four stop and two glide finals reconstructible to Proto-Ong-Be (see Table 32). There is no voicing or aspiration contrast in this position-all stop finals are unreleased and voiceless, and all nasal and glide finals are voiced. Unlike initials, these finals did not trigger further tonal splits after tones became phonemic in pre-Proto-Ong-Be. The reconstruction of Proto-Ong-Be finals is quite straightforward, since all proto-finals have identical reflexes in all varieties surveyed.

TABLE 32 Proto-Ong-Be finals

\begin{tabular}{llllll}
\hline & bilabial & alveolar & palatal & velar & glottal \\
\hline stops & $*-\mathrm{p}$ & ${ }^{*}-\mathrm{t}$ & & ${ }^{*}-\mathrm{k}$ & $*-1$ \\
nasals & $*-\mathrm{m}$ & ${ }^{-} \mathrm{n}$ & & ${ }^{*} \mathrm{n}$ & \\
glides & $*-\mathrm{w}$ & & ${ }^{-\mathrm{j}}$ & & \\
& & & & & \\
\hline
\end{tabular}

Table 33 illustrates word sets with $-m$ as their final. Internal evidence supports the reconstruction of *-m as the obvious reconstruction for this correspondence.

\begin{tabular}{|c|c|c|c|c|c|c|c|}
\hline Chinese & English & CL & $\mathbf{Y X}$ & $\mathbf{L T}$ & LSG & HT & $\mathbf{X Y}$ \\
\hline & & $-\mathbf{m}$ & $-\mathbf{m}$ & $-\mathbf{m}$ & $-\mathbf{m}$ & $-\mathbf{m}$ & $-\mathbf{m}$ \\
\hline 抬 & 'to lift' & $\operatorname{ham}^{1}$ & $\operatorname{ham}^{1}$ & $\operatorname{ham}^{1}$ & $\operatorname{ham}^{1}$ & ham $^{1}$ & $\operatorname{ham}^{1}$ \\
\hline 蛋 & ‘egg’ & $\mathrm{n}^{1} \mathrm{~m}^{1}$ & nam $^{1}$ & nem ${ }^{1}$ & num $^{1}$ & num $^{1}$ & zum $^{1}$ \\
\hline
\end{tabular}




\begin{tabular}{|c|c|c|c|c|c|c|c|}
\hline Chinese & English & CL & $\mathbf{Y X}$ & LT & LSG & HT & $\mathbf{X Y}$ \\
\hline & & $-\mathbf{m}$ & $-\mathbf{m}$ & $-\mathbf{m}$ & $-\mathbf{m}$ & $-\mathbf{m}$ & $-\mathbf{m}$ \\
\hline 苦 & 'bitter' & $\mathrm{kam}^{2}$ & $\mathrm{kam}^{2}$ & $\mathrm{kam}^{2}$ & $\mathrm{kam}^{2}$ & $\mathrm{kam}^{2}$ & $\mathrm{kam}^{2}$ \\
\hline 低 & 'low' & dom ${ }^{3}$ & dom $^{3}$ & dom ${ }^{3}$ & dom $^{3}$ & đวัm ${ }^{3}$ & dom ${ }^{3}$ \\
\hline 晚上 & 'night' & $\mathrm{kim}^{4}$ & kom $^{4}$ & kom $^{4}$ & - & $\mathrm{kom}^{4}$ & $\mathrm{kom}^{4}$ \\
\hline 舔 & 'to lick' & - & $\lim ^{4}$ & $\operatorname{zim}^{4}$ & $\lim ^{4}$ & $\lim ^{4}$ & $\lim ^{4}$ \\
\hline
\end{tabular}

All the words in Table 34 end in $-n$. For this reason, ${ }^{*}-n$ is reconstructed as the final for this correspondence.

\begin{tabular}{|c|c|c|c|c|c|c|c|}
\hline Chinese & English & CL & $\mathbf{Y X}$ & $\mathbf{L T}$ & LSG & HT & $\mathbf{X Y}$ \\
\hline & & $-n$ & $-n$ & $-n$ & $-n$ & $-n$ & $-n$ \\
\hline 孫 & 'grandchild' & $\operatorname{lan}^{1}$ & $\operatorname{lan}^{1}$ & $\operatorname{lan}^{1}$ & $\operatorname{lan}^{1}$ & $-\operatorname{lan}^{1}$ & $-\operatorname{lan}^{1}$ \\
\hline 雨 & 'rain' & $\mathrm{p}^{\mathrm{h}} \mathrm{an}^{1}$ & $\mathrm{p}^{\mathrm{h}} \mathrm{On}^{1}$ & pfen $^{1}$ & fun $^{1}$ & fun $^{1}$ & $\mathrm{p}^{\mathrm{h}} \mathrm{un}^{1}$ \\
\hline 房屋 & 'house' & $\operatorname{zan}^{2}$ & $\operatorname{zan}^{2}$ & $\operatorname{zan}^{2}$ & $\operatorname{zan}^{2}$ & $\operatorname{lan}^{2}$ & $\operatorname{zan}^{2}$ \\
\hline 熱 & 'hot; to heat' & $\operatorname{lun}^{3}$ & $\operatorname{lun}^{3}$ & $\operatorname{lun}^{3}$ & $\operatorname{lun}^{3}$ & $\operatorname{lun}^{3}$ & $\operatorname{lun}^{3}$ \\
\hline 汗水 & 'sweat' & hen $^{4}$ & hon 4 & hon 4 & hon $^{4}$ & hon 4 & hon 4 \\
\hline
\end{tabular}

Table 35 presents a list of words that end in a velar nasal. Hence, ${ }^{*}-\eta$ is reconstructed here.

\begin{tabular}{|c|c|c|c|c|c|c|c|}
\hline Chinese & English & CL & $\mathbf{Y X}$ & LT & LSG & HT & $\mathbf{X Y}$ \\
\hline & & $-\eta$ & $-\eta$ & $-\eta$ & $-\eta$ & $-\eta$ & $-\eta$ \\
\hline 亮 & 'bright (not dark)' & 6ay ${ }^{1}$ & bay $^{1}$ & 6ay ${ }^{1}$ & bay $^{1}$ & bay ${ }^{1}$ & bay $^{1}$ \\
\hline 水蛭 & 'aquatic leech' & $-6 i \eta^{1}$ & $-6 i \eta^{1}$ & $-6 i 1^{1}$ & $\operatorname{bin}^{1}$ & $\operatorname{bin}^{1}$ & $\operatorname{bin}^{1}$ \\
\hline 篩子 & 'winnowing basket' & $\mathrm{zo \eta}^{2}$ & $\mathrm{zon}^{2}$ & $\mathrm{zJ \eta}^{2}$ & $\mathrm{zon}^{2}$ & $\operatorname{lon} \eta^{2}$ & $\operatorname{lo\eta } \eta^{2}$ \\
\hline 鹹 & 'salty' & $\operatorname{zan}^{3}$ & $\mathrm{za \eta}^{3}$ & $\operatorname{zan}^{3}$ & $\operatorname{zan}^{3}$ & $\operatorname{la\eta }^{3}$ & $\operatorname{la\eta }^{3}$ \\
\hline 稻草 & 'straw' & mun 4 & $\operatorname{muy}^{4}$ & $\mathrm{mug}^{4}$ & mug $^{4}$ & mug $^{4}$ & mur $^{4}$ \\
\hline
\end{tabular}

Because all the words in Table 36 end with a bilabial stop, ${ }^{*}$-p is the most suitable candidate for the proto-final. 
TABLE $36 \quad *-p$

\begin{tabular}{|c|c|c|c|c|c|c|c|}
\hline Chinese & English & CL & $\mathbf{Y X}$ & LT & LSG & HT & $\mathbf{X Y}$ \\
\hline & & $-p$ & $-p$ & $-p$ & $-p$ & $-p$ & $-p$ \\
\hline 挑(擔) & $\begin{array}{l}\text { 'to carry with a shoulder } \\
\text { pole' }\end{array}$ & hap $^{7}$ & hap $^{7}$ & hap $^{7}$ & hap $^{7}$ & hap $^{7}$ & hap $^{7}$ \\
\hline 生(肉) & 'raw' & $\mathrm{zop}^{7}$ & $\mathrm{zop}^{7}$ & $\mathrm{zsp}^{7}$ & zәр ${ }^{7}$ & $\operatorname{zip}^{7}$ & $\operatorname{lip}^{7}$ \\
\hline 睡、躺 & 'to sleep; to lie down' & $\operatorname{lap}^{7}$ & $\operatorname{lap}^{7}$ & $\operatorname{lap}^{7}$ & $\operatorname{lap}^{7}$ & lap ${ }^{7}$ & lap $^{7}$ \\
\hline 指甲 & 'nail' & ziap $^{8}$ & zip $^{8}$ & zep $^{8}$ & zip $^{8}$ & $\operatorname{lip}^{8}$ & $\operatorname{lip}^{8}$ \\
\hline 米 & 'husked rice' & zap $^{8}$ & zap 8 & zep $^{8}$ & zop ${ }^{8}$ & zop $\mathrm{p}^{8}$ & lэp 8 \\
\hline
\end{tabular}

Table 37 consists of words that end in - $t$. All the modern reflexes retain the original final, so *-t is assigned to this set.

\begin{tabular}{|c|c|c|c|c|c|c|c|}
\hline Chinese & English & CL & $\mathbf{Y X}$ & LT & LSG & HT & $\mathbf{X Y}$ \\
\hline & & $-t$ & $-t$ & $-t$ & $-t$ & $-t$ & $-t$ \\
\hline 冷 & 'cold' & nit $^{7}$ & nit 7 & nit $^{7}$ & nit $^{7}$ & nit $^{7}$ & nit $^{7}$ \\
\hline 韌 & 'pliable but strong' & nat $^{7}$ & nat $^{7}$ & nat $^{7}$ & nat $^{7}$ & nat $^{7}$ & zat $^{7}$ \\
\hline 頭蟲 & 'head louse' & $\mathrm{kat}^{7}$ & kat $^{7}$ & $\mathrm{~kat}^{7}$ & $\mathrm{~kat}^{7}$ & $\mathrm{~kat}^{7}$ & kat $^{7}$ \\
\hline 刀子 & 'knife' & $\mathrm{mit}^{8}$ & $\mathrm{mit}^{8}$ & mit $^{8}$ & $\mathrm{mit}^{8}$ & $\mathrm{mit}^{8}$ & mit $^{8}$ \\
\hline 萆類 & 'mushroom' & $\begin{array}{l}\text { lot }^{8} \\
\text { hot }^{8}\end{array}$ & hot $^{8}$ & hat $^{8}$ & hət $^{8}$ & hit $^{8}$ & hit $^{8}$ \\
\hline
\end{tabular}

In Table 38 , all the Ong-Be words end in $-k$. Therefore, it is best to reconstruct *-k for this set.

\begin{tabular}{|c|c|c|c|c|c|c|c|}
\hline Chinese & English & CL & $\mathbf{Y X}$ & LT & LSG & HT & $\mathbf{X Y}$ \\
\hline & & $-k$ & $-k$ & $-k$ & $-k$ & $-k$ & $-k$ \\
\hline 洗(手) & 'to wash (hands)' & $\operatorname{tuk}^{7}$ & $\operatorname{tuk}^{7}$ & $\operatorname{tuk}^{7}$ & $\operatorname{tuk}^{7}$ & $\operatorname{tuk}^{7}$ & $\operatorname{tuk}^{7}$ \\
\hline 寺頭 & 'taro' & $\operatorname{sak}^{7}$ & $\operatorname{sak}^{7}$ & $\operatorname{sak}^{7}$ & $\operatorname{sak}^{7}$ & $\operatorname{sak}^{7}$ & $\operatorname{sak}^{7}$ \\
\hline 織布機 & 'loom' & $d_{\varepsilon \mathrm{k}^{8}}$ & $d \varepsilon \mathrm{k}^{8}$ & $d \varepsilon \mathrm{k}^{8}$ & - & đək ${ }^{8}$ & đək ${ }^{8}$ \\
\hline 兒女 & 'offspring' & $\mathrm{l} \varepsilon \mathrm{k}^{8}$ & $\mathrm{l} \varepsilon \mathrm{k}^{8}$ & $\mathrm{l} \varepsilon \mathrm{k}^{8}$ & $\operatorname{lək}^{8}$ & $\operatorname{lək}^{8}$ & $\operatorname{lək}^{8}$ \\
\hline 鼻涕 & 'mucus' & $\mathrm{muk}^{8}$ & juk $^{8}-\mathrm{i}$ & $\operatorname{mok}^{8}$ & $\mathrm{muk}^{8}$ & $\mathrm{muk}^{8}$ & $\mathrm{muk}^{8}$ \\
\hline
\end{tabular}


Table 39 presents a list of words that have a glottal stop as the final. I thus reconstruct * $?$ for this sound correspondence given that all the examples have a glottal stop coda. Note that there are not many words in Ong-Be that end in a glottal stop.

TABLE 39 *-?

\begin{tabular}{|c|c|c|c|c|c|c|c|}
\hline Chinese & English & CL & $\mathbf{Y X}$ & $\mathbf{L T}$ & LSG & HT & $\mathbf{X Y}$ \\
\hline & & $-?$ & $-?$ & $-?$ & $-?$ & $-?$ & $-?$ \\
\hline 魚鱗 & 'fish scale' & $\operatorname{lu} P^{7}$ & $\operatorname{li} P^{7}$ & $\operatorname{li}^{7}$ & li $P^{8}-t$ & $\operatorname{lip}^{7}$ & $\operatorname{li}{ }^{7}$ \\
\hline 螞蟻 & 'ant' & $\operatorname{mup}^{8}$ & $\operatorname{mup}^{8}$ & mor' & $\operatorname{mup}^{8}$ & $\operatorname{mup}^{8}$ & $\operatorname{mup}^{8}$ \\
\hline 血 & 'blood' & $6 a P^{7}$ & ba? ${ }^{7}$ & ba? ${ }^{7}$ & $6 a ?^{8}-t$ & ba? ${ }^{7}$ & ba? ${ }^{7}$ \\
\hline 硬 & 'firm; hard' & zuaP $^{7}$ & zua? $^{7}$ & zua? $^{7}$ & zuaP7 $^{7}$ & luar ${ }^{7}$ & lua?7 \\
\hline 針 & 'needle' & ga? 8 & ya? 8 & ya? ${ }^{8}$ & ga? ${ }^{8}$ & ya?8 & ya? ${ }^{8}$ \\
\hline 吞、嚥 & 'to swallow' & ləP? & $\operatorname{li} P^{7}$ & lip ${ }^{7}$ & ləP? & lə? ${ }^{7}$ & - \\
\hline
\end{tabular}

Two glides, $-j$ and $-w$, can serve as codas as well. Because all the modern data in Tables $40-41$ consist of $-j$ and $-w$ respectively, ${ }^{*}$ j and ${ }^{*}-\mathrm{w}$ must be reconstructed for Proto-Ong-Be.

TABLE $40 \quad *_{-j}$

\begin{tabular}{|c|c|c|c|c|c|c|c|}
\hline Chinese & English & CL & $\mathbf{Y X}$ & $\mathbf{L T}$ & LSG & HT & $\mathbf{X Y}$ \\
\hline & & $-\mathbf{j}$ & $-\mathbf{j}$ & $-\mathbf{j}$ & $-\mathrm{j}$ & $-\mathbf{j}$ & $-\mathbf{j}$ \\
\hline 死 & 'to die; dead' & daj $^{1}$ & daj $^{1}$ & daj $^{1}$ & daj $^{1}$ & daj $^{1}$ & daj $^{1}$ \\
\hline 雞 & 'chicken’ & $\mathrm{kaj}^{1}$ & $\mathrm{k} \varepsilon j^{1}$ & $\mathrm{kej}^{1}$ & $\mathrm{kaj}^{1}$ & $\mathrm{kaj}^{1}$ & $\mathrm{kaj}^{1}$ \\
\hline 火 & 'fire' & $6 \varepsilon j^{2}$ & $v \varepsilon j^{2}$ & $v_{e j}^{2}$ & vəj $j^{2}$ & vəj ${ }^{2}$ & vəj ${ }^{2}$ \\
\hline 扇子 & 'fan' & $\mathrm{p}^{\mathrm{h}} \mathrm{aj}{ }^{2}$ & $\mathrm{p}^{\mathrm{h}} \mathrm{\jmath j} \mathrm{j}^{2}$ & $p f j^{2}$ & $f \partial j^{2}$ & $f \partial j^{2}$ & $\mathrm{p}^{\mathrm{h}} \mathrm{\jmath j}{ }^{2}$ \\
\hline 問 & 'to ask' & tej ${ }^{3}$ & toj $j^{3}$ & toj $j^{3}$ & $\mathrm{t} \mathrm{j}^{3}$ & $\mathrm{t} 0 \mathrm{j}^{3}$ & $\mathrm{t} \mathrm{j}^{3}$ \\
\hline 䧳性 & female & $\mathrm{maj}^{4}$ & $\mathrm{maj}^{4}$ & $\mathrm{maj}^{4}$ & $\mathrm{maj}^{4}$ & $\mathrm{maj}^{4}$ & $\mathrm{maj}^{4}$ \\
\hline
\end{tabular}

TABLE $41 \quad *-w$

\begin{tabular}{|c|c|c|c|c|c|c|c|}
\hline Chinese & English & CL & $\mathbf{Y X}$ & LT & LSG & HT & $\mathbf{X Y}$ \\
\hline & & $-w$ & $-w$ & $-w$ & $-w$ & $-\mathbf{w}$ & $-w$ \\
\hline 彎曲 & 'to bend' & Pew $^{1}$ & - & Pew $^{1}$ & $P \varepsilon W^{1}$ & $P \varepsilon w^{1}$ & $P \varepsilon w^{1}$ \\
\hline 尿 & 'urine' & zow $^{1}$ & $z^{2} w^{1}$ & zew $^{1}$ & $\mathrm{ZOW}^{1}$ & ZOW $^{1}$ & low $w^{1}$ \\
\hline 虾蚓 & 'earthworm' & new $^{2}$ & nวw ${ }^{2}$ & nวw $^{2}$ & now ${ }^{2}$ & now $^{2}$ & nวw ${ }^{2}$ \\
\hline
\end{tabular}




\begin{tabular}{|c|c|c|c|c|c|c|c|}
\hline Chinese & English & CL & $\mathbf{Y X}$ & LT & LSG & HT & $\mathbf{X Y}$ \\
\hline & & $-w$ & $-w$ & $-\mathbf{w}$ & $-w$ & $-w$ & $-w$ \\
\hline 吠 & 'to bark' & saw $^{3}$ & saw $^{3}$ & $\mathrm{saw}^{3}$ & saw $^{3}$ & saw $^{3}$ & saw $^{3}$ \\
\hline 吹 & 'to blow' & bew 4 & vวw ${ }^{4}$ & vow $^{4}$ & vow $^{4}$ & vow 4 & vวw $^{4}$ \\
\hline
\end{tabular}

\section{$5 \quad$ Approaching Ong-Be Initials and Finals in Light of Kra-Dai}

\subsection{Ong-Be Tones}

Ong-Be varieties spoken around Haikou generally have more tones than those spoken in Lingao County. Zhang et al. (1985) and Liang \& Zhang (1997) show that Longtang, and Longqiao of Qiongshan (now part of Haikou) have nine tones $\left(1,1^{\prime}, 2,3,4,7,7^{\prime}, 8\right.$, and $\left.8^{\prime}\right)$, whereas the varieties in Lingao have only six tones $(1,2,3,4,7$, and 8). They recognize six basic tone categories (Tones $1,2,3,4,7$, and 8) in Ong-Be languages, four of which are non-checked tones $(1,2,3$, and 4$)$ and two of which are checked tones $(7$ and 8$)$, which only occur in syllables ending in a stop coda. Considering that Ong-Be tones show regular correspondence across varieties, tone categories are reconstructed in this study. Gedney (1989: 67-68) also talks about the reconstruction of tone categories for Proto-Tai, and reaches a conclusion that, regardless of how these categories are ordered, tones in Tai languages alone support the reconstruction of Tai tone categories. ${ }^{13}$

Contemporary Ong-Be tone data are labelled following the order used in Zhang et al. (1985) and Liang \& Zhang (1997). There is also a letter system where A, B, C, and D are used to categorize tone across Kra-Dai languages. The mapping between the numeric system and the letter system as well as the correspondence between Ong-Be tones and those of the other Kra-Dai languages has been demonstrated in Zhang et al. (1985), Hansell (1988), Liang \& Zhang (1997), and Ostapirat (2000). A synopsis of the tone correspondences is presented in Table 42. Note that Ong-Be shows no distinction between Kra-Dai Tone B and Tone C.

TABLE 42 Ong-Be tonal categories in a Kra-Dai perspective

\begin{tabular}{|l|l|l|l|l|l|l|l|}
\hline $\begin{array}{l}\text { Tone } \\
\text { Category }\end{array}$ & Chinese & English & CL & LT & LSG & HT & XY \\
\hline $\begin{array}{l}\text { Kra-Dai } \\
\text { Tone A }\end{array}$ & 魚 & 'fish' & $6 a^{1}$ & $6 a^{1}$ & $6 a^{1}$ & $6 a^{1}$ & $6 a^{1}$ \\
\cline { 2 - 8 } & 水田 & 'paddy field' & $\mathrm{nia}^{2}$ & $\mathrm{nia}^{2}$ & $\mathrm{nia}^{2}$ & $\mathrm{nia}^{2}$ & $\mathrm{nia}^{2}$ \\
\hline \multirow{2}{*}{$\begin{array}{l}\text { Kra-Dai } \\
\text { Tone B }\end{array}$} & 吠 & 'to bark' & $\mathrm{saw}^{3}$ & $\mathrm{saw}^{3}$ & $\mathrm{saw}^{3}$ & $\mathrm{saw}^{3}$ & $\mathrm{saw}^{3}$ \\
\cline { 2 - 8 } & 吹 & 'to blow' & $6 \mathrm{fw}^{4}$ & $\mathrm{vow}^{4}$ & $\mathrm{vow}^{4}$ & $\mathrm{vow}^{4}$ & $\mathrm{vow}^{4}$ \\
\hline
\end{tabular}




\begin{tabular}{|l|l|l|l|l|l|l|l|}
\hline $\begin{array}{l}\text { Tone } \\
\text { Category }\end{array}$ & Chinese & English & CL & LT & LSG & HT & XY \\
\hline \multirow{2}{*}{$\begin{array}{l}\text { Kra-Dai } \\
\text { Tone C }\end{array}$} & 臉 & 'face' & $\mathrm{na}^{3}$ & $\mathrm{na}^{3}$ & $\mathrm{na}^{3}$ & $\mathrm{na}^{3}$ & $\mathrm{na}^{3}$ \\
\cline { 2 - 8 } & 水 & 'water' & $\mathrm{nam}{ }^{4}$ & $\mathrm{nam}^{4}$ & $\mathrm{nam}^{4}$ & $\mathrm{nam}^{4}$ & $\mathrm{nam}^{4}$ \\
\hline \multirow{2}{*}{$\begin{array}{l}\text { Kra-Dai } \\
\text { Tone D }\end{array}$} & 翅膀 & 'wing' & $6 \mathrm{fk}^{7}$ & $6 \mathrm{ik}^{7}$ & $6 \mathrm{ik}^{7}$ & $\mathrm{bik}^{7}$ & $6 \mathrm{fik}^{7}$ \\
\cline { 2 - 8 } & 刀子 & 'knife' & $\mathrm{mit}^{8}$ & $\mathrm{mit}^{8}$ & $\mathrm{mit}^{8}$ & $\mathrm{mit}^{8}$ & $\mathrm{mit}^{8}$ \\
\hline
\end{tabular}

Haudricourt (1954a; 1954b) demonstrates that tonal splits in Vietnamese were conditioned by the voicing of initials, a model of which is also applicable to tonal splits in Kra-Dai and Sinitic languages. Since the number of tones in Ong-Be ranges from six to nine, one might wonder how these tones developed from four basic Kra-Dai tone categories and what conditioned the splits of tones. Hansell (1988) shows that, although discrepancies are observed, there is a correlation between Ong-Be tones and Proto-Tai voicing in conjunction with tone series. Table 43 presents examples illustrating the voicing in initials of Ong-Be, Tai, Kam-Sui, and Hlai. Tones 1, 2, 3, 4, 7 and 8 in Ong-Be are rendered as $\mathrm{A} 1, \mathrm{~A} 2, \mathrm{BC} 1, \mathrm{BC} 2, \mathrm{D} 1$, and $\mathrm{D} 2$ respectively.

When the initials of Proto-Tai are referred to, we see that the early voicing contrast caused earlier KraDai tones (Tones A, B, C, and D) to further split into two series, i.e., series 1 (Tones A1, BC1, and D1) and series 2 (Tones A2, BC2, and D2), in Ong-Be and that compared to Proto-Kam-Sui, Ong-Be tones associated with the voicing of initials show a closer connection to the voicing in Proto-Tai. Take 'paddy field' for instance. Ong-Be has $/ \mathrm{nia}^{2} /$ the tone of which is in series 2, indicating an early voiced initial at the time of the split. Proto-Tai has *n- which agrees with Ong-Be tones in terms of the voicing. By contrast, ProtoKam-Sui has *?r- and Proto-Hlai (Norquest 2007) has *hn-, suggesting a voiceless initial at the time of the split. It is noteworthy that such information conceived in Ong-Be tones can only be apprehended when external evidence is consulted.

TABLE 43 Ong-Be tones in a Kra-Dai perspective ${ }^{14}$

\begin{tabular}{|c|c|c|c|c|c|c|c|c|c|}
\hline $\begin{array}{l}\text { Kra-Dai Tone } \\
\text { Category }\end{array}$ & $\begin{array}{l}\text { Ong-Be } \\
\text { Tone }\end{array}$ & Chinese & English & LT & PT (1977) & PT (2009) & PKS (1988) & PH (2004) & PH (2007) \\
\hline \multirow[t]{5}{*}{ Tone A1 } & \multirow[t]{3}{*}{1} & 魚 & 'fish' & $6 a^{1}$ & *pl- & *pl- & *p- & *al- & *hl- \\
\hline & & 死 & 'to die' & daj $^{1}$ & ${ }^{*} \operatorname{tr}-$ & ${ }^{*} \mathrm{p}-\mathrm{t}-$ & *pj- & - & - \\
\hline & & 耳朵 & 'ear' & $\mathrm{sa}^{1}$ & ${ }^{*} \mathrm{xr}-$ & *kr- & *khr- & - & - \\
\hline & \multirow[t]{2}{*}{$1^{\prime}$} & 飛 & 'to fly' & $6 e n^{1}$ & $* 2 b-$ & ${ }^{*} 6-$ & - & *(?)b- & ${ }^{*} 6-$ \\
\hline & & 吃 & 'to eat' & kon $^{1}$ & *k- & ${ }^{*} \mathrm{k}-$ & ${ }^{*} \mathrm{c}-$ & - & ${ }^{*} \mathrm{k}^{\mathrm{h}_{-}}$ \\
\hline \multirow[t]{3}{*}{ Tone A2 } & \multirow[t]{3}{*}{2} & 火 & 'fire' & $\mathrm{v \varepsilon j} j^{2}$ & ${ }^{*} \mathrm{~V}-$ & ${ }^{*} \mathrm{~W}-$ & ${ }^{*} \mathrm{pw}-$ & *ap- & *f- \\
\hline & & 水田 & 'paddy field' & $\operatorname{nia}^{2}$ & *n- & *n- & *?r- & *an- & *hn- \\
\hline & & 茅草 & 'thatch grass' & $\mathrm{tia}^{2}$ & ${ }^{*} \mathrm{\gamma}^{-}$ & ${ }^{*} \mathrm{\gamma}^{-}$ & - & *ik- & ${ }^{*} \mathrm{Cih}-$ \\
\hline
\end{tabular}

14 PT (Proto-Tai) data are from Li (1977) and Pittayaporn (2009), PKs (Proto-Kam-Sui) data from Thurgood (1988), and PH (Proto-Hali) data from Ostapirat (2004) and Norquest (2007). 


\begin{tabular}{|c|c|c|c|c|c|c|c|c|c|}
\hline $\begin{array}{l}\text { Kra-Dai Tone } \\
\text { Category }\end{array}$ & $\begin{array}{l}\text { Ong-Be } \\
\text { Tone }\end{array}$ & Chinese & English & $\mathbf{L T}$ & PT (1977) & PT (2009) & PKS (1988) & PH (2004) & PH (2007) \\
\hline \multirow[t]{3}{*}{ Tone BC1 } & \multirow[t]{3}{*}{3} & 舊 & 'old (not new)' & $\mathrm{kaw}^{3}$ & *k- & ${ }^{*} \mathrm{k}-$ & *k- & ${ }^{*} \mathrm{k}-$ & $* \mathrm{k}^{\mathrm{h}_{-}}$ \\
\hline & & 吠 & 'to bark' & saw $^{3}$ & *hr- & ${ }^{*} \mathrm{~h} r-$ & *khr- & - & - \\
\hline & & 肩膀 & 'shoulder' & $6 i^{3}$ & *2b- & ${ }^{*} \mathrm{C}-\mathrm{b}-$ & - & *au- & ${ }^{*} \mathrm{v}-$ \\
\hline \multirow[t]{3}{*}{ Tone $\mathrm{BC} 2$} & \multirow[t]{3}{*}{4} & 水 & 'water' & nam $^{4}$ & ${ }^{*} \mathrm{nl} / \mathrm{r}-$ & ${ }^{*} \mathrm{C}-\mathrm{n}-$ & $* \mathrm{n}-$ & $* n-$ & ${ }^{*} \mathrm{C}-\mathrm{n}-$ \\
\hline & & 雲 & 'cloud'd & $6 a^{4}$ & ${ }^{*} \mathrm{v}-$ & ${ }^{*} \mathrm{v}-$ & * $\mathrm{Xw}-$ & *ap- & $*_{\mathrm{f}}-$ \\
\hline & & 髹子 & 'beard' & moy $^{4}$ & *m- & *m- & ${ }^{*} \mathrm{~m}-$ & *(ə)m- & *hm- \\
\hline \multirow[t]{6}{*}{ Tone D1 } & \multirow[t]{3}{*}{7} & 嘴 & 'mouth' & $6 \mathrm{ak}^{7}$ & *p- & *p- & *p- & - & - \\
\hline & & 簏 & 'to sun-dry' & $\mathrm{dak}^{7}$ & ${ }^{*} \mathrm{pr}-$ & ${ }^{*} \mathrm{p}-\mathrm{r}-$ & - & - & - \\
\hline & & 胸膛 & 'chest' & $-30 k^{7}$ & *?- & *?- & ${ }^{*} \mathrm{t}-$ & - & - \\
\hline & \multirow[t]{3}{*}{$7^{\prime}$} & 滿 & 'full, filled' & $\mathrm{dit}^{7}$ & *th- & *k-t- & ${ }^{*} \mathrm{t}-$ & $*_{\mathrm{t}-}$ & $*_{\mathrm{t}}-$ \\
\hline & & 疏菜 & 'vegetable' & $\mathrm{sak}^{7}$ & ${ }^{*} \mathrm{phl} / \mathrm{r}-$ & *pr- & - & - & - \\
\hline & & 餓 & 'hungry' & $\mathrm{zak}^{7}$ & ${ }^{*} \mathrm{hj}-$ & *?j- & ${ }^{*} 6 j-$ & - & - \\
\hline \multirow[t]{5}{*}{ Tone D2 } & \multirow[t]{2}{*}{8} & 繩索 & 'rope' & $\mathrm{dak}^{8}$ & *y- & $*^{*}$ & *?l- & - & - \\
\hline & & 烏鴉 & 'crow' & $-\mathrm{Pak}^{8}$ & *?- & *?- & - & *?- & *?- \\
\hline & \multirow[t]{3}{*}{$8^{\prime}$} & 蜈蚣 & 'centipede' & zep ${ }^{8}$ & "khr- & * $\mathrm{q}-\mathrm{s}-$ & *khry- & *ar- & ${ }^{*} \Gamma-$ \\
\hline & & 洗 & 'to wash' & $\mathrm{dek}^{8}$ & ${ }^{*} \mathrm{z}-$ & ${ }^{*} \mathrm{z}-$ & ${ }^{*} \mathrm{z}-$ & ${ }^{*} \mathrm{~s}-$ & *s- \\
\hline & & 馬 & 'horse' & $\mathrm{maP}^{8}$ & *m- & *m- & ${ }^{*} \mathrm{~m}-$ & - & - \\
\hline
\end{tabular}

d 'Cloud' in Ong-Be corresponds to 'sky' in Tai (Hansell 1988: 247).

As shown in Table $44,{ }^{*} \mathrm{Cr}$ - and ${ }^{*} \mathrm{C} r$ - of Proto-Kra-Dai have $t s^{-2}$ and $s^{-1}$, respectively as their reflexes in Ong-Be. In Tai and Kam-Sui, the voicing of the first segment in a consonant cluster at the time of the tonal splits plays an important role, as illustrated in Table 44 where consonant clusters are reconstructible to Proto-Tai and Proto-Kam-Sui. Unlike Siamese (Tai) and Sui (Kam-Sui), early clusters left no segmental traces in Ong-Be of them once being a cluster (also see Table 57).

TABLE $44 \quad{ }^{15}$ Voicing and consonant clusters

\begin{tabular}{|c|c|c|c|c|c|c|c|c|c|}
\hline Chinese & English & PKD & Ров & LT & PT (1977) & PT (2009) & Siamese & PKS (1988) & Sui \\
\hline 芋頭 & 'taro' & *pr- & ${ }^{*} \mathrm{~s}^{1-}$ & $\mathrm{sak}^{7}$ & ${ }^{*} \mathrm{phl} / \mathrm{r}-$ & ${ }^{*} \mathrm{pr}-$ & $\mathrm{p}^{\mathrm{h}} \mathrm{uək}^{\mathrm{DL} 1}$ & ${ }^{*} 6 r-$ & ?ya:k ${ }^{7}$ \\
\hline 吠 & 'to bark' & ${ }^{*} \mathrm{kr}-$ & ${ }^{*} s^{1-}$ & saw $^{3}$ & *hr- & ${ }^{*} \mathrm{~h}_{\mathrm{r}}-$ & haw ${ }^{B 1}$ & ${ }^{*} \mathrm{k}^{\mathrm{h}} \mathrm{r}-$ & $\mathrm{k}^{\mathrm{h}} \mathrm{au}^{5}$ \\
\hline
\end{tabular}

15 PKD stands for Proto-Kra-Dai from Ostapirat (2005b) and РОв for Proto-Ong-Be proposed in this research. 


\begin{tabular}{|c|c|c|c|c|c|c|c|c|c|}
\hline Chinese & English & PKD & Ров & LT & PT (1977) & PT (2009) & Siamese & PKS (1988) & Sui \\
\hline 明天 & 'tomorrow' & *br- & ${ }^{*} \mathrm{ts}^{2}{ }_{-}$ & $\mathrm{tsck}^{8}$ & *vr- & ${ }^{*} \mathrm{jm}-\mathrm{r}-$ & $\mathrm{p}^{\mathrm{h}} \mathrm{ruj}^{\mathrm{B} 2}-\mathrm{f}$ & - & - \\
\hline 呻吟 & 'to moan' & *gr- & $* \mathrm{ts}^{2}$ & $\operatorname{tsa}^{2}(\mathrm{~L} \& \mathrm{Z})$ & ${ }^{*} \mathrm{k}^{\mathrm{h}_{-}}$ & *gr- & $\mathrm{k}^{\mathrm{h}} \mathrm{ra}: \mathrm{\eta}^{\mathrm{A} 2}$ & - & - \\
\hline
\end{tabular}

However, suprasegmental change (tone changes) and segmental change may have occurred at different times. There are at least two possible paths of a secondary tonal split conditioned by voicing. In the hypothetical path illustrated in example (1), early clusters ${ }^{* *}$ pr- $^{\mathrm{A}}$ and ${ }^{* *} \mathrm{br}^{-\mathrm{A}}$ with Tone A first simplify to two affricates, ${ }^{*}$ ts- ${ }^{\mathrm{A}}$ and ${ }^{*} \mathrm{~d}{ }_{-}{ }^{\mathrm{A}}$ accompanied by redundant pitch differences conditioned by the voicing in initials, and further lose the voicing contrast, which results in an affricate, $t s$-, with two more phonemic tones (A1 and $\left.A_{2}\right)$ in step 3. Another possibility is shown in the hypothetical path in example (2) where early clusters with Tone A accompanied by redundant pitch differences conditioned by the voicing in initials first lose the voicing contrast, resulting in two more phonemic tones (A1 and A2) in step 2, before the clusters further simplify to an affricate, $t s$-, in step 3. In the latter path, the suprasegmental change, i.e., the tonal split (Tone $\mathrm{A} \rightarrow$ Tones $\mathrm{A} 1$ and A2), predates segmental change $\left({ }^{*}-\mathrm{r}->t s^{-}\right)$. Because none of today's Ong-Be varieties preserve a voicing contrast, it is unclear whether tonal splits associated with an early voicing is inherited from the Proto-Ong-Be or a pre-Proto-Ong-Be stage.

(1)

$$
\begin{aligned}
& { }^{*}{ }^{*} \mathrm{pr}^{-}{ }^{\mathrm{A}}>{ }^{*} \text { ts- }^{\mathrm{A}}>\text { ts- }{ }^{\mathrm{Al}} \\
& { }^{*}{ }^{*} \mathrm{br}^{-}{ }^{-}>{ }^{*} \mathrm{Ct}^{-}{ }^{\mathrm{A}}>\mathrm{ts}^{-}{ }^{\mathrm{A}} 2 \\
& \text { (2) }{ }^{*} \text { pr- }^{-}{ }^{A}{ }^{*} \text { pr- }^{-A 1}>\text { ts- }{ }^{-A 1} \\
& { }^{*}{ }^{*} \mathrm{br}^{-}{ }^{\mathrm{A}}>{ }^{*} \mathrm{pr}^{-\mathrm{A} 2}>\text { ts }^{-{ }^{A} 2}
\end{aligned}
$$

Li (1977) faced the same dilemma in his reconstruction of Proto-Tai, considering that the early voicing contrast had already been lost in a large number of Tai languages. He states that "It is perhaps safe to assume that the Proto-Tai tones A, B, C, and D split into two series, those with a voiceless initial and those with a voiced initial ... It is not known exactly when the two series became phonemic, and it is conceivable that the dates may vary according to dialects." (pp. 25-26). However, Thai scripts developed in the $13^{\text {th }}$ century preserved indications of the earlier voicing contrast, and several scholars have pointed out that Cao Bang retains a voicing contrast (see Haudricourt 1949, 1960, 1961; L-Thongkum 1997; Hoàng 1997; Pittayaporn 2009; among others). Both serve as convincing evidence for the reconstruction of Proto-Tai voicing associated with initials. In reconstructing Proto-Tai, Pittayaporn (2009: 17) also emphasizes that the use of external evidence such as data from other Kra-Dai languages together with typology can only serves as "additional support for reconstructions proposed on the basis of Tai-internal evidence." Taken together, an indication of the voicing contrast, but not the voicing per se, is supported by the Ong-Be internal evidence, even though the association of an early voicing contrast with Ong-Be tones has to be interpreted in light of Kra-Dai.

In addition to the tonal splits induced by an early voicing contrast, Longtang shows extra tonal splits in Tone D (7, 7', 8, and 8') and Tone A (1 and 1'). Liang \& Zhang (1997) attribute these tonal splits to a vowel length contrast, and suggest that the tonal split in Tone $\mathrm{A} 1$ is a parallel development to the tonal splits in Tone Di because they share the same pitches (Tone $7=13=$ Tone 1 ; Tone $7^{\prime}=44=$ Tone ${ }^{\prime}$ ). Such splits are attested in the eastern Ong-Be subgroup, including Longqiao, Longquan, Yongxing, and Shishan (but not Changliu). Based on Liang \& Zhang's proposal, Chen (2018) further proposes that the splits in Tone D1 and Tone D2 in Longtang, Longqiao, and Longquan, and Tone D1 in Yongxing and Shishan are triggered 
by the vowel length distinction at the time of the tonal splits, not identical to that of Proto-Ong-Be (see Table 45).

TABLE 45 Further tonal splits in Longtang and Yongxing

\begin{tabular}{|c|c|c|c|c|c|c|c|}
\hline Tone Category & Ong-Be Tone & Chinese & English & $\mathbf{C L}$ & $\mathbf{Y X}$ & LT & $\begin{array}{l}\text { РОВ } \\
\text { (Chen 2018) }\end{array}$ \\
\hline \multirow[t]{4}{*}{ Tone A1 } & 1 & 魚 & 'fish' & $6 a^{35}$ & $6 a^{21(3)}$ & $6 a^{13}$ & *ba:A1 \\
\hline & & 死 & 'to die' & daj ${ }^{35}$ & daje21(3) & daj ${ }^{13}$ & *da:j ${ }^{\mathrm{Al}}$ \\
\hline & & 耳朵 & ‘ear' & $\mathrm{sa}^{35}$ & $\mathrm{sa}^{21(3)}$ & $\mathrm{sa}^{13}$ & *sa:A1 \\
\hline & & 重 & 'heavy' & $\mathrm{k}^{\mathrm{h}} \mathrm{en}^{35}$ & $\mathrm{k}^{\mathrm{h}} \mathrm{\partial n}^{21(3)}$ & $\mathrm{k}^{\mathrm{h}} \mathrm{Jn}^{13}$ & ${ }^{*} \mathrm{k}^{\mathrm{h}} \mathrm{n}^{\mathrm{Al}}$ \\
\hline \multirow[t]{3}{*}{ Tone A1 } & $1^{\prime}$ & 月亮 & 'moon' & saj $^{35}$ & $\mathrm{saj}^{21(3)}$ & sej $^{44}$ & * saj $^{\mathrm{Al}}$ \\
\hline & & 飛 & 'to fly' & $\operatorname{ban}^{35}$ & $6 \mathrm{n}^{21(3)}$ & $6 e n^{44}$ & ${ }^{*} \operatorname{vin}^{\mathrm{A} 1}$ \\
\hline & & 吃 & 'to eat' & kon $^{35}$ & $k^{21(3)}$ & kon $^{44}$ & *kən ${ }^{\mathrm{A} 1}$ \\
\hline \multirow[t]{4}{*}{ Tone D1 } & 7 & 嘴 & 'mouth' & 6ak 55 & $6 a k^{21}$ & $6 a k^{13}$ & *6a:k ${ }^{\mathrm{D} 1}$ \\
\hline & & 曬 & 'to sun-dry' & dak ${ }^{55}$ & $\mathrm{dak}^{21}$ & dak $k^{13}$ & *da:k ${ }^{\mathrm{D} 1}$ \\
\hline & & 踩 & 'to step on' & đว่ $\mathrm{k}^{55}$ & đวk $\mathrm{k}^{21}$ & đつ. $\mathrm{k}^{13}$ & * ${ }^{2} \mathrm{k}^{\mathrm{D} 1}$ \\
\hline & & 胸膛 & 'chest' & - Puak $^{55}$ & $-\mathrm{PJk}^{21}$ & $-\mathrm{Pok}^{13}$ & * $\mathrm{Puk}^{\mathrm{D} 1}$ \\
\hline \multirow[t]{4}{*}{ Tone D1 } & $7^{\prime}$ & 滿 & 'full, filled' & $\mathrm{dik}^{55}$ & dik ${ }^{55}$ & $\mathrm{dit}^{44}$ & *di:k $\mathrm{k}^{\mathrm{D} 1}$ \\
\hline & & 疏菜 & 'vegetable' & $\operatorname{sak}^{55}$ & $\operatorname{sak}^{55}$ & $\mathrm{sak}^{44}$ & * $\operatorname{sak}^{\mathrm{D} 1}$ \\
\hline & & 餓 & 'hungry' & $\mathrm{zak}^{55}$ & ziak $^{55}$ & $\mathrm{zak}^{44}$ & ${ }^{*} \mathrm{zak}^{\mathrm{D} 1}$ \\
\hline & & 打 & 'to hit' & $\mathrm{kit}^{55}$ & $\mathrm{kit}^{55}$ & $\mathrm{kit}^{44}$ & *ki:t $\mathrm{t}^{\mathrm{D} 1}$ \\
\hline \multirow[t]{6}{*}{ Tone D2 } & 8 & 繩索 & 'rope' & dak $^{21}$ & dak ${ }^{33}$ & dak ${ }^{21}$ & *da:k $\mathrm{k}^{\mathrm{D} 2}$ \\
\hline & & 烏鴉 & 'crow' & $-\mathrm{Pak}^{21}$ & $-\mathrm{Pak}^{33}$ & $-\mathrm{Pak}^{21}$ & * $\mathrm{Pa}: \mathrm{k}^{\mathrm{D} 2}$ \\
\hline & & 鹿 & ‘deer’ & $\mathrm{ts}^{\mathrm{h}} \mathrm{uak}^{21}$ & $\mathrm{ts}^{\mathrm{h}} \mathrm{ok}^{33}$ & $\mathrm{ts}^{(\mathrm{h})} \mathrm{Ok}^{21}$ & ${ }^{*} \operatorname{tsuk}^{\mathrm{D} 2}$ \\
\hline & $8^{\prime}$ & 蜈蚣 & 'centipede' & $\mathrm{zJp}^{21}$ & $\mathrm{zop}^{33}$ & zep $^{33}$ & ${ }^{*} \operatorname{zip}^{\mathrm{D} 2}$ \\
\hline & & 洗 & 'to wash' & $\mathrm{dak}^{21}$ & dak 33 & $\mathrm{dek}^{33}$ & *dak $\mathrm{k}^{\mathrm{D} 2}$ \\
\hline & & 馬 & 'horse' & $\operatorname{mar}^{21}$ & $\operatorname{maP}^{33}$ & $\operatorname{ma} P^{33}$ & *maPD2 \\
\hline
\end{tabular}

\subsection{Proto-Ong-Be Initials Revisited}

Based on comparisons of Ong-Be with other Kra-Dai languages, Proto-Ong-Be initials can be divided into two subcategories when tones are taken into consideration. As presented in Table 46 , initials associated with Ong-Be odd-number tones (Tones 1, 1', 3, 7, and $7^{\prime}$ ) are labelled with a raised ${ }^{1}$ to indicate that they were voiceless at the time of the split. On the other hand, initials with even-number tones (Tones $2,4,8$, and $\left.8^{\prime}\right)$ are labelled using a raised ${ }^{2}$, indicating an association with earlier voiced initials. In addition, voicing contrasts can be found in sonorants as well. Tones indicating voiceless sonorants might result from earlier consonant clusters at the time of the splits. 
TABLE 46 Proto-Ong-Be initials with tone series

\begin{tabular}{|c|c|c|c|c|c|c|c|c|c|c|}
\hline \multirow{3}{*}{$\begin{array}{l}\text { implosives } \\
\text { stops }\end{array}$} & \multicolumn{2}{|c|}{ bilabial } & labiodental & \multicolumn{2}{|c|}{ alveolar } & \multicolumn{2}{|c|}{ alveo-palatal } & \multicolumn{2}{|l|}{ velar } & \multirow[t]{2}{*}{ glottal } \\
\hline & ${ }^{*} 6^{1}$ & $* 6^{2}$ & & ${ }^{*} d^{1}$ & $* d^{2}$ & & & & & \\
\hline & ${ }^{*} \mathrm{p}^{\mathrm{hl}}$ & ${ }^{*} \mathrm{p}^{\mathrm{h} 2}$ & & $* \mathrm{t}^{1}$ & $* \mathrm{t}^{2}$ & & & ${ }^{*} \mathrm{k}^{1}$ & ${ }^{*} \mathrm{k}^{2}$ & $* P^{1}$ \\
\hline & & & & & & & & ${ }^{*} \mathrm{k}^{\mathrm{h} 1}$ & ${ }^{*} \mathrm{k}^{\mathrm{h} 2}$ & \\
\hline nasals & ${ }^{*} \mathrm{~m}^{1}$ & ${ }^{*} \mathrm{~m}^{2}$ & & ${ }^{*} \mathrm{n}^{1}$ & $* \mathrm{n}^{2}$ & $* \mathrm{n}^{1}$ & ${ }^{*} \mathrm{n}^{2}$ & $* y^{1}$ & $* \eta^{2}$ & \\
\hline affricates & & & & $* \mathrm{ts}^{1}$ & $* \mathrm{ts}^{2}$ & & & & & \\
\hline \multirow[t]{2}{*}{ fricatives } & & & $* \mathrm{v}^{2}$ & ${ }^{*} \mathrm{~S}^{1}$ & & ${ }^{*} \mathrm{z}^{1}$ & ${ }^{*} \mathrm{z}^{2}$ & & & ${ }^{*} \mathrm{~h}^{1} \quad * \mathrm{~h}^{2}$ \\
\hline & & & & ${ }^{*} \mathrm{z}^{1}$ & ${ }^{*} \mathrm{z}^{2}$ & & & & & \\
\hline laterals & & & & $*^{1}$ & $*{ }^{2}$ & & & & & \\
\hline
\end{tabular}

There are two potential proto initials that require more attention. In Table 19, the tone of ' $\operatorname{crow}^{\prime} / \mathrm{Pak}^{8} /$ which happens to be in series 2 must be due to a sporadic tone change because $?$ - with Tone D2 is nonrecurrent. Also, due to physiological constraints, it is impossible to reconstruct a voiced glottal stop, because to produce a glottal stop the glottis is closed, but to voice a consonant the glottis must be open. In addition, 'crow' corresponds to series 1 in the rest of the Kra-Dai languages, i.e., ${ }^{*} \mathrm{ak}^{\mathrm{D}}$ in Proto-Kra, *?a: $\mathrm{k}^{\mathrm{D}}$ in Proto-Hlai, both pointing to a proto voiceless initial. The forms for 'chopsticks' (from 箸; Middle Chinese: *tř o) in Table 21 have tone 4, belonging to series 2, leading us to expect * ${ }^{2}$ - for this initial. However, ${ }^{*} \mathrm{~s}^{2}$ - is not reconstructed mainly because it does not have a recurrent sound correspondence. The reason ${ }^{*} \mathrm{~s}^{2}$ - is missing will be discussed in connection with Table 52 .

\subsection{From Proto-Kra-Dai to Proto-Ong-Be Initials and Finals}

Ostapirat $(2005 \mathrm{~b})$ demonstrates how Proto-Kra-Dai obstruents are reflected in contemporary Ong-Be varieties, including how Proto-Ong-Be evolved into today's Ong-Be. If we synthesize Ostapirat (2005b) with my proposed reconstruction, Proto-Kra-Dai stop initials *p-, *b-, *t-, *d-, and *k- are reflected as $/ b^{1}-/, / b^{2}-/, / d^{1}-/, / d^{2}-/, / k^{1}-/$, and $/ k^{2}-/$ in today's Ong-Be. To put it another way, the Proto-Kra-Dai voicing contrast in each pair has been neutralized at the segmental level in Ong-Be, although such neutralization is compensated for in tone (see Table 47).

Proto-Kra-Dai voiced bilabial and alveolar stops and their voiceless counterparts are now voiced implosives $/ 6^{1}-/, / 6^{2}-/, / d^{1}-/$ and $/ d^{2}-/$ in Ong-Be languages. The development of implosives 6 - and $d$ has been reported to be an areal feature in Guangdong (廣東), Guangxi (廣西), Hainan, and Vietnam (Solnit 1982). ${ }^{16}$ In the given examples, the information conceived in Proto-Ong-Be tones agrees with the voicing of the initials in Proto-Tai and Proto-Kam-Sui, except for 'leaf' and 'navel' where Ong-Be tones indicate an early voiced initial.

16 Edmondson \& Esling (2006) states that the so-called "preglottalized stops" (or "implosives" in other literature) are true implosives in Vietnamese, but glottalized stops in Tai languages. Regardless of the phonetic nature of these voiced stops, preglottalized stops and implosives are not phonemically contrastive with each other in Ong-Be. 
TABLE 47 Proto-Kra-Dai stop initials

\begin{tabular}{|c|c|c|c|c|c|c|c|c|}
\hline Chinese & English & PKD & PT (1977) & PT (2009) & PKS (1988) & РОв & LT & $\mathbf{X Y}$ \\
\hline 魚 & fish & *p- & *pl- & *pl- & *p- & ${ }^{*} 6^{1-}$ & $6 a^{1}$ & $6 a^{1}$ \\
\hline 嘴 & mouth & ${ }^{*} \mathrm{p}-$ & ${ }^{*} \mathrm{p}-$ & *p- & ${ }^{*} \mathrm{p}-$ & *61- & $6 \mathrm{ak}^{7}$ & $6 a k^{7}$ \\
\hline 葉 & leaf & *b- & * $\mathrm{Pb}-$ & $* 6-$ & ${ }^{*} \mathrm{pw}-$ & $* 6^{2}$ & $6 o^{2}$ & $6 o^{2}$ \\
\hline 肝 & liver & $* t-$ & $* t-$ & $* t-$ & - & ${ }^{*} d^{1-}$ & - & dəp ${ }^{7}$ \\
\hline 掉落 & to fall & $* t-$ & $* t-$ & ${ }^{*} \mathrm{t}-$ & - & ${ }^{*} d^{1-}$ & dok ${ }^{7}$ & dok ${ }^{7}$ \\
\hline 肚臍 & navel & $* \mathrm{~d}-$ & ${ }^{*} \mathrm{Pbl} / \mathrm{r}-$ & ${ }^{*} d w-$ & ${ }^{*}$ blw- & $* d^{2}$ & $-\mathrm{lo}^{2} ;-\mathrm{zJ}^{2}$ & $-d \partial^{2}$ \\
\hline 雞 & chicken & ${ }^{*} \mathrm{k}-$ & ${ }^{*} \mathrm{k}-$ & ${ }^{*} \mathrm{k}-$ & ${ }^{*} \mathrm{k}-$ & ${ }^{*} \mathrm{k}^{1}-$ & $\mathrm{kej}^{1}$ & kaj $^{1}$ \\
\hline 舊 & old (thing) & *k- & ${ }^{*} \mathrm{k}-$ & ${ }^{*} \mathrm{k}-$ & *k- & ${ }^{*} \mathrm{k}^{1-}$ & $\mathrm{kaw}^{3}$ & kaw $^{3}$ \\
\hline
\end{tabular}

Ostapirat (2005b: 279) points out that the Proto-Kra-Dai voiceless (post)velar stop medials had spirantized to * $\mathrm{z}$ - (intervocalic voicing) before they further strengthened to * $\mathrm{g}$-, cf. Proto-Kra-Dai voiceless velar initial *k- which remains as $/ \mathrm{k}^{1}-/$ (see the last two examples in Table 47 ). Later, early voiced and voiceless velar stops merged at the segmental level (but with different reflexes manifested in the tones), becoming voiceless stops $/ \mathrm{k}^{1}-/$ and $/ \mathrm{k}^{2}-/$ in contemporary Ong-Be (see Table 48 ). As discussed in Maddieson (2013), it is harder to maintain a voicing contrast at the back of the throat. It is not surprising, then, that the early * $\mathrm{g}$ - has devoiced and became $/ \mathrm{k}^{2}-/$. For these two Kra-Dai etyma with voiceless (post) velar stop medials, even though Proto-Ong-Be implies an early voiced initial, Proto-Tai (Li 1977) and Proto-Kam-Sui (1988) both indicate a voiceless initial at the time of the tonal split. Proto-Tai reconstructed by Pittayaporn (2009), on the other hand, points to a voiced initial when the split occurred, which agrees with the suprasegmental features in my proposed reconstruction.

TABLE 48 Proto-Kra-Dai-K-17

\begin{tabular}{lllllllll}
\hline Chinese & English & PKD & PT (1977) & PT (2009) & PKs (1988) & POв & LT & XY \\
\hline 苦 & 'bitter' & ${ }^{*}-\mathrm{K}-$ & ${ }^{*} \mathrm{X}^{-}$ & ${ }^{*} \mathrm{C}-\mathrm{q}-$ & ${ }^{*} \mathrm{k}-$ & ${ }^{*} \mathrm{k}^{2-}$ & $\mathrm{kam}^{2}$ & $\mathrm{kam}^{2}$ \\
屎 & 'excrement' & ${ }^{*}-\mathrm{K}-$ & ${ }^{*} \mathrm{X}^{-}$ & ${ }^{*} \mathrm{C}-\mathrm{q}-$ & ${ }^{*} \mathrm{k}-$ & ${ }^{*} \mathrm{k}^{2-}$ & $\mathrm{kaj}^{4}$ & $\mathrm{kaj}^{4}$
\end{tabular}

In my reconstruction, in addition to ${ }^{*} \mathrm{~d}^{1}$ - and ${ }^{*} \mathrm{~d}^{2}{ }_{-}$, Proto-Ong-Be has another coronal series ${ }^{*} \mathrm{t}^{1}$ - and ${ }^{*} \mathrm{t}^{2-}$ which originated from palatalized (post)velar stops and affricates in Proto-Kra-Dai (see Table 49). As aforementioned, Proto-Tai has lost the environment of palatalization, whereas Ong-Be and Kam-Sui still retain traces of it. It is now clear why ${ }^{*} \mathrm{t}^{1}$ - and ${ }^{*} \mathrm{t}^{2}$ - in Proto-Ong-Be are reflected as voiceless stops (siding with the velars), given that the reflexes of ${ }^{*} k^{1}$ - and ${ }^{*} k^{2}$ - are voiceless. This piece of evidence shows that implosivization took place before depalatalization as proposed in Ostapirat (2005b). That is, ${ }^{*} \mathrm{~d}^{1}$ - and ${ }^{*} \mathrm{~d}^{2}{ }^{2}$ must have become voiced implosives in Ong-Be before ${ }^{*} \mathrm{k}^{\mathrm{j}}{ }^{1}$ and ${ }^{*} \mathrm{k}^{\mathrm{j}}{ }^{2}$ - depalatalized to dental/alveolar stops, ${ }^{*} \mathrm{t}^{1}$ - and ${ }^{*} \mathrm{t}^{2}$-. Otherwise ${ }^{*} \mathrm{t}^{1}$ - and ${ }^{*} \mathrm{t}^{2}$ - would have merged with ${ }^{*} \mathrm{~d}^{1}$ - and ${ }^{*} \mathrm{~d}^{2}{ }^{2}$. It also reveals that

17 The Proto-Kra-Dai data used for comparison are after Ostapirat (2005b). K stands for Proto-Kra-Dai (post)velar stop medials*-k- or *-q- (pp. 281-282). 
Proto-Ong-Be stop initials reformed voicing according to place of articulation of the pre-Proto-Ong-Be stop onsets $\left({ }^{*} \mathrm{~d}^{1}{ }^{1-}\right.$, ${ }^{*} \mathrm{~d}^{2}$ - and bilabials imploded, while ${ }^{*} \mathrm{k}^{1}$ - and ${ }^{*} \mathrm{k}^{2}$ ( (and ${ }^{*} \mathrm{t}^{1-}$ and $\left.{ }^{*} \mathrm{t}^{2}-\right)$ devoiced).

TABLE 49 Proto-Kra-Daipalatalized obstruents

\begin{tabular}{|c|c|c|c|c|c|c|c|c|}
\hline Chinese & English & PKD & PT (1977) & PT (2009) & PKS (1988) & РОВ & $\mathbf{L T}$ & $\mathbf{X Y}$ \\
\hline 回答 & 'to reply' & ${ }^{*} \mathrm{C}-\mathrm{K}^{\mathrm{j}}-$ & ${ }^{*} \mathrm{X}-$ & ${ }^{*} \chi^{-}$ & - & $* \mathrm{t}^{1-}$ & $\tan ^{1}$ & $\tan ^{1}$ \\
\hline 烏鴉 & 'to crow' & ${ }^{*} \mathrm{C}_{\mathrm{o}}-\mathrm{K}^{\mathrm{j}}-$ & ${ }^{*} \mathrm{X}-$ & ${ }^{*} \chi^{-}$ & - & $* \mathrm{t}^{1-}$ & $\tan ^{1}$ & $\tan ^{1}$ \\
\hline 問 & 'to ask' & ${ }^{*} \mathrm{ts}^{\mathrm{j}}-$ & - & - & - & $* \mathrm{t}^{1-}$ & toj $j^{3}$ & toj $j^{3}$ \\
\hline 尾巴 & 'tail' & $* t \int-$ & - & - & *khj- & $* \mathrm{t}^{1-}$ & $\operatorname{tur}^{7}$ & tur ${ }^{7}$ \\
\hline 茅草 & 'thatch grass' & ${ }^{*}-\mathrm{K}^{\mathrm{j}}-$ & ${ }^{*} \mathrm{\gamma}^{-}$ & ${ }^{*} \mathrm{X}^{-}$ & - & $* \mathrm{t}^{2}$ & $\operatorname{tia}^{2}$ & $\operatorname{tia}^{2}$ \\
\hline
\end{tabular}

These sound changes, as suggested in Ostapirat (2005b: 281-283), possibly passed through intermediate ${ }^{*} t_{\text {- }}$ and ${ }^{*} \mathrm{~d}_{\mathrm{b}}$ - before merging into ${ }^{*} \mathrm{t}$-, which later lost palatalization, resulting in $/ \mathrm{t}^{1}-/$ and $/ \mathrm{t}^{2}-/$. Again, because none of the Ong-Be reflexes shed light on early palatalization, it is impossible to reconstruct a palatalized segment and past voicing contrast in Ong-Be without referring to external evidence.

As for the contemporary fricative initials, such as $/ \mathrm{v}^{1}-|,| \mathrm{v}^{2}-|,| \mathrm{p}^{\mathrm{h}} \mathrm{1}_{-}-,\left|\mathrm{z}^{1}-\right|,\left|\mathrm{z}^{2}-\right|$ and $/ \mathrm{h}^{1}-\mid$, many of them originated from Proto-Kra-Dai medial stops, as illustrated in Table 50 (see Ostapirat 2005b for a detailed discussion on Proto-Kra-Dai to Ong-Be, and Michaud 2012 on the development of spirantization of medial stops in today's monosyllabic languages). While Proto-Tai and Proto-Kam-Sui preserve signs of consonant clusters, Proto-Ong-Be shows no traces of these complex onsets. Since all the Kra-Dai complex onsets have been simplified in Ong-Be, it is impossible to reconstruct such a system using the comparative method due to lack of supporting evidence.

TABLE 50 Proto-Kra-Dai stop medials

\begin{tabular}{|c|c|c|c|c|c|c|c|c|}
\hline Chinese & English & PKD & PT (1977) & PT (2009) & PKS (1988) & РОв & LT & $\mathbf{X Y}$ \\
\hline 局膀 & 'shoulder' & ${ }^{*} \mathrm{C}-\mathrm{p}-$ & *Pb- & ${ }^{*} \mathrm{C}-\mathrm{b}-$ & - & ${ }^{*} \mathrm{v}^{1-}$ & $-6 i^{3}$ & $-v_{i a}^{3}$ \\
\hline 飛 & 'to fly' & ${ }^{*} \mathrm{C}-\mathrm{p}-$ & *?b- & ${ }^{*} 6-$ & - & ${ }^{*} \mathrm{v}^{1-}$ & $6 e n^{1}$ & $\operatorname{vin}^{1}$ \\
\hline 火 & 'fire' & ${ }^{*}-\mathrm{p}-$ & ${ }^{*} \mathrm{v}-$ & ${ }^{*} \mathrm{~W}-$ & - & ${ }^{*} \mathrm{v}^{2-}$ & $\mathrm{vej}^{2}$ & $\operatorname{vəj}^{2}$ \\
\hline 米糠 & 'chaff' & ${ }^{*}-\mathrm{p}-$ & - & - & - & ${ }^{*} \mathrm{v}^{2}$ & $\mathrm{v} 0^{4}$ & $\mathrm{v} 0^{4}$ \\
\hline 雨 & 'rain' & ${ }^{*} \mathrm{k}-\mathrm{p}-$ & *f- & ${ }^{*} \mathrm{C}-\mathrm{w}-$ & ${ }^{*} \mathrm{Xw}-$ & ${ }^{*} \mathrm{p}^{\mathrm{h} 1_{-}}$ & pfen ${ }^{1}$ & $\mathrm{p}^{\mathrm{h}} \mathrm{un}^{1}$ \\
\hline 小米 & 'millet' & ${ }^{*} \mathrm{k}-\mathrm{p}-$ & $* \mathrm{f}-$ & ${ }^{*} \mathrm{~h}_{\mathrm{W}}-$ & - & ${ }^{*} \mathrm{p}^{\mathrm{h} 1_{-}}$ & $v^{2} \eta^{4-t}$ & $\mathrm{p}^{\mathrm{h}} \mathrm{a} \eta^{3}$ \\
\hline 生 & 'raw' & ${ }^{*} \mathrm{C}-\mathrm{d}-$ & ${ }^{*} \mathrm{Pdl} / \mathrm{r}-$ & ${ }^{*} \mathrm{C}-\mathrm{d}-$ & ${ }^{*} d-$ & ${ }^{*} z^{1-}$ & $\mathrm{zop}^{7}$ & $\operatorname{lip}^{7}$ \\
\hline 鼻 & 'nose' & ${ }^{*} \mathrm{C}-\mathrm{d}-$ & *?d- & $* d-$ & ${ }^{*} \mathrm{Pn}-$ & ${ }^{*} \mathrm{z}^{1-}$ & $\mathrm{zoy}^{1}$ & $\operatorname{lo\eta } \eta^{1}$ \\
\hline 船 & 'boat' & ${ }^{*} \mathrm{C} / \mathrm{V}-\mathrm{d}-$ & $* \mathrm{dr}-$ & ${ }^{*} \mathrm{C}-\mathrm{r}-$ & - & ${ }^{*} \mathrm{Z}^{2-}$ & zua $^{4}$ & $\operatorname{lua}^{4}$ \\
\hline 樹根 & 'root'e & ${ }^{*} \mathrm{C} / \mathrm{V}-\mathrm{d}-$ & $* \mathrm{dr}-$ & ${ }^{*} \mathrm{C}-\mathrm{r}-$ & - & ${ }^{*} \mathrm{z}^{2-}$ & ziak $^{8}$ & $\operatorname{liak}^{8}$ \\
\hline $\begin{array}{l}\text { 挑 } \\
\text { (擔) }\end{array}$ & $\begin{array}{l}\text { 'to carry on a } \\
\text { pole' }\end{array}$ & ${ }^{*} \mathrm{k}-\mathrm{t}-$ & *thr- & $* \operatorname{tr}-$ & - & ${ }^{*} \mathrm{~h}^{1-}$ & hap $^{7}$ & hap $^{7}$ \\
\hline 抬 & $\begin{array}{l}\text { 'to carry by two } \\
\text { people' }\end{array}$ & ${ }^{*} \mathrm{k}-\mathrm{t}-$ & *thr- & $* \operatorname{tr}-$ & - & ${ }^{*} \mathrm{~h}^{1-}$ & ham $^{1}$ & ham $^{1}$ \\
\hline
\end{tabular}

e In the western Ong-Be subgroup, the Kra-Dai word for 'root' is preserved in the lexical item Ficus microcarpa (a type of banyan tree with small sized leaves). 
The Proto-Ong-Be alveo-palatal fricative ${ }^{*} \mathrm{z}^{1}$ - and the velar affricate ${ }^{*} \mathrm{k}^{\mathrm{h} 1}$ - in Table $5^{1}$ were derived from Proto-Kra-Dai voiced fricatives. They are now reflected as $/ \mathrm{z}^{1}-/$ and $/ \mathrm{kx}^{1}-/ \sim / \mathrm{k}^{\mathrm{h}} \mathrm{I}_{-} /$in the eastern Ong-Be subgroup, but $/ \mathrm{z}^{1}-/$ and $/ \mathrm{kx}^{1}-/ \sim / \mathrm{k}^{\mathrm{h}}{ }_{-} /$or $/ \mathrm{x}^{1}-/$ in the western Ong-Be subgroup. It is clear that this affricate in Ong-Be results from the collapse of Proto-Kra-Dai di- or sesquisyllabicity, where the minor syllable onset clustered with the major syllable onset, as exemplified in 'heavy' and 'light (not heavy)'. By contrast, the early pretonic syllable onset in 'medicine' left no reconstructible traces at the segmental level in contemporary Ong-Be, although the Ong-Be tone indicates that an earlier form of 'medicine' contains a voiceless initial at the time of the split, cf. the voicing and the complex onset of 'medicine' in Proto-Kam-Sui.

TABLE 51 Proto-Kra-Dai voiced fricatives

\begin{tabular}{|c|c|c|c|c|c|c|c|c|}
\hline Chinese & English & PKD & PT (1977) & PT (2009) & PKS (1988) & РОВ & $\mathbf{L T}$ & $\mathbf{X Y}$ \\
\hline 站 & 'to stand' & ${ }^{*} 3^{-}$ & *Pj- & ${ }^{*} \mathrm{C}-\mathrm{y}-$ & - & ${ }^{*} Z^{1}$ & $\operatorname{zun}^{1}$ & $\operatorname{zun}^{1}$ \\
\hline 餓 & 'hungry' & ${ }^{*} 3^{-}$ & ${ }^{*} \mathrm{Pj}-$ & $*{ }^{*} \mathbf{j}$ & ${ }^{*} 6 j--i$ & ${ }^{*} \mathrm{Z}^{1}{ }_{-}$ & $\mathrm{zak}^{7}$ & ziak $^{7}$ \\
\hline 藥 & 'medicine' & ${ }^{*} \mathrm{k}-\mathrm{d}_{3}-$ & $* ? \mathrm{j}-$ & *? $\mathrm{j}-$ & *gj- & ${ }^{*} \mathrm{z}^{1-}$ & $\mathrm{zia}^{1}$ & $\mathrm{zia}^{1}$ \\
\hline 重 & 'heavy' & ${ }^{*} \mathrm{~K}-\mathrm{z}-$ & - & - & - & ${ }^{*} \mathrm{k}^{\mathrm{h} 1_{-}}$ & $\mathrm{kx}^{1} \mathrm{n}^{1}$ & $\mathrm{k}^{\mathrm{h}} \mathrm{On}^{1}$ \\
\hline 輕 & 'light (weight)' & ${ }^{*} \mathrm{~K}-\mathrm{z}-$ & - & - & - & ${ }^{*} \mathrm{k}^{\mathrm{h} 1}$ & $\mathrm{kxo}^{3}$ & $\mathrm{k}^{\mathrm{h}} \mathrm{\jmath}^{3}$ \\
\hline
\end{tabular}

Table $5^{2}$ shows that the Proto-Ong-Be initials * ${ }^{1}$ - and *ts²- were derived from Proto-Kra-Dai consonant clusters containing *-r-. The data below clearly demonstrate that the Proto-Kra-Dai proto voiceless initials have led to tone series 1 and the proto voiced initials have developed into series 2 in today's Ong-Be languages. Compared to Proto-Ong-Be, initials in Proto-Tai and Proto-Kam-Sui retain more clues regarding earlier consonant clusters.

\begin{tabular}{|c|c|c|c|c|c|c|c|c|}
\hline Chinese & English & PKD & PT (1977) & PT (2009) & PKS (1988) & Ров & $\mathbf{L T}$ & $\mathbf{X Y}$ \\
\hline 芋頭 & 'taro' & ${ }^{*} \mathrm{Pr}-$ or Kr- & *phl/r- & *pr- & *6r- & $* s^{1-}$ & $\operatorname{sak}^{7}$ & $\mathrm{sak}^{7}$ \\
\hline 吠 & 'to bark' & *Pr- or Kr- & *hr- & *hr- & *khr- & ${ }^{*} \mathrm{~s}^{1-}$ & $\mathrm{saw}^{3}$ & $\mathrm{saw}^{3}$ \\
\hline 路 & 'road' & ${ }^{*} \mathrm{Pr}-$ or Kr- & ${ }^{*} \mathrm{xr}-$ & ${ }^{*} \mathrm{hr}-$ & - & ${ }^{*} \mathrm{~s}^{1-}$ & $\operatorname{sen}^{1}$ & $\operatorname{sun}^{1}$ \\
\hline 明天 & 'tomorrow' & ${ }^{*} \mathrm{Br}-\mathrm{or}{ }^{*} \mathrm{Gr}-$ & *vr- & ${ }^{*} \mathrm{jm}-\mathrm{r}$ & - & $* \mathrm{ts}^{2}-$ & $\mathrm{tsck}^{8}$ & $\mathrm{ts}^{\mathrm{h}} \partial \mathrm{k}^{8}$ \\
\hline 泫 & 'wet' & ${ }^{*} \mathrm{Br}-\mathrm{or}{ }^{*} \mathrm{Gr}-$ & - & - & *?r- & $* \mathrm{ts}^{2}-$ & $\mathrm{ts}^{\mathrm{h}} \mathrm{ek}^{8}$ & tsak $^{8}$ \\
\hline
\end{tabular}

18 The capital P stands for a proto voiceless bilabial initial, B for a proto voiced bilabial initial, and G for a proto voiced (post) velar initial. 
One might wonder about the development of Proto-Ong-Be * ${ }^{2}$ - and *ts ${ }^{1}$ - from a Kra-Dai perspective. Many words given in Table 26 such as 'chopsticks', 'kitchen range', 'indigo', 'to drop', and 'deer', although reconstructible using internal evidence for ${ }^{*} t^{1}{ }^{1}$, are early Chinese loans borrowed no later than ProtoOng-Be split off. These words are not reconstructible to the Proto-Kra-Dai level because they are not found in primary subgroups which were not in contact with Chinese. Note that, as mentioned earlier, Ong-Be $s$-, originating from Proto-Kra-Da *-r-, corresponds to Jizhao Haihua ts-. Based on Ostapirat (1998: $341)$, the earlier form of my postulated ${ }^{*} \mathrm{~s}^{1}$ - could be an affricate.

Table 53 presents an inverted reconstruction of the Proto-Ong-Be initial system based on Ostapirat (2005b; 2017, p.c.). Ostapirat (2005b: 288-289) proposes four specific changes, which took place in chronological order, to account for the sound changes from Proto-Kra-Dai voiceless medial stops to today's Ong-Be initials. They are (1) lenition (intervocalic voicing \& spirantization of voiceless medial stops), (2) occlusion (the outputs from the previous step became stops or affricates, except for labiodentals), (3) devoicing (for velar stops, including palatalized velars which later became palatals) and implosivization (for alveolar and labial stops), and (4) depalatalization (for palatals).

TABLE 53 An inverted reconstruction ${ }^{19}$ of Proto-Ong-Be initials (not exhaustive)

\begin{tabular}{|c|c|c|c|c|c|c|c|c|c|c|c|}
\hline \multirow[b]{2}{*}{ stops } & \multicolumn{2}{|c|}{ bilabial } & \multicolumn{2}{|c|}{ labiodental } & \multicolumn{2}{|c|}{ alveolar } & \multicolumn{2}{|c|}{ (alveo)palatal } & \multicolumn{2}{|c|}{ velar } & glottal \\
\hline & *p & *b & & & $*_{\mathrm{t}}$ & *d & $*_{\mathrm{t}}$ & ${ }^{*} \mathrm{~d}_{0}$ & ${ }^{*} \mathrm{k}$ & *g & *? \\
\hline nasals & $* \mathrm{~m}$ & *m & & & ${ }^{*} \mathrm{n}$ & *n & $* \stackrel{\circ}{\rho}$ & *n & * $\supseteqq$ & $* \eta$ & \\
\hline affricates & & & & & *ts & & & $* \mathrm{~d}$ & & & \\
\hline fricatives & & & *u & *v & *s & & & $* 3$ & ${ }^{*} \mathrm{x}$ & ${ }^{*} \mathrm{X}$ & *h \\
\hline liquids & & & & & *lo & *l & ${ }^{*} \mathrm{r}$ & $* \mathrm{r}$ & & & \\
\hline
\end{tabular}

Based on internal evidence, I have reconstructed nine Proto-Ong-Be finals. It is noteworthy that ProtoKra-Dai had two other finals, *-l and *-t, not reconstructible in Proto-Ong-Be. Table 54 demonstrates that Proto-Kra-Dai *-l has merged with *-n in Proto-Ong-Be. The final *-t, according to Ostapirat (2009: 53), "assimilated with the preceding vowel" in Ong-Be. As for the Proto-Kra-Dai final *-c, it is regularly reflected in Ong-Be as a glottal stop, which I accordingly reconstruct as * $-2 .{ }^{20}$ Unlike Proto-Ong-Be, which retains a distinction between Proto-Kra-Dai *-t and *-c while merging *-l with *-n, Proto-Tai still distinguishes Proto-Kra-Dai final *-n from *-l, and *-t from *-c. Proto-Kam-Sui, on the other hand, has merged Proto-Kra-Dai *-c with *-t, and ${ }^{*}-1$ with ${ }^{*}-n$.

19 An inverted reconstruction is to assign phonetic values to a place-holder "from above", when internal evidence does not allow certain features to be reconstructed (see Anttila 1989: 346 and Fox 1995: 88-89 for more details). For example, different accentuations in Proto-Germanic can only be determined using Proto-Indo-European.

20 Note that there are multiple sources of Proto-Ong-Be *-?. In addition to being a reflex of Proto-Kra-Dai final *-c, as in 'fish scale', 'ant', and 'blood', one of the reviewers points out that in Yongxing and Linshigang, this glottal stop can be a reduced form of $-t$, the sound change of which is common in Kra-Dai languages. Some of the Ong-Be etyma that end in this glottal stop codas are lexical innovations unreconstructible beyond the Ong-Be branch, such as 'needle' and 'to swallow'. 
TABLE 54 Proto-Kra-Dai ${ }^{*}-$ land ${ }^{*}-c$

\begin{tabular}{|c|c|c|c|c|c|c|c|c|}
\hline Chinese & English & PKD & РT (1977) & PT (2009) & PKS (1988) & Ров & LT & $\mathrm{XY}$ \\
\hline 吃 & 'to eat' & ${ }^{*}-\mathrm{n}$ & ${ }^{*}-n$ & ${ }^{*}-\mathrm{n}$ & ${ }^{*}$-n & ${ }^{*}-\mathrm{n}$ & $k^{\prime} n^{1}$ & $\mathrm{kon}^{1}$ \\
\hline 舌頭 & 'tongue' & ${ }^{*}-\mathrm{n}$ & ${ }^{*}-\mathrm{n}$ & ${ }^{*}-\mathrm{n}$ & - & ${ }^{*}-\mathrm{n}$ & $\operatorname{lin}^{4}$ & $\operatorname{lin}^{4}$ \\
\hline 穿山甲 & 'pangolin' & ${ }^{*}-1$ & ${ }^{*}-\mathrm{n}$ & ${ }^{*}-1$ & ${ }^{*}-\mathrm{n}$ & ${ }^{*}-\mathrm{n}$ & hən ${ }^{4}$ & $\mathrm{hin}^{4}$ \\
\hline 石頭 & 'stone' & ${ }^{*}-1$ & ${ }^{*}-\mathrm{n}$ & ${ }^{*}-1$ & - & ${ }^{*}-\mathrm{n}$ & $\mathrm{din}^{2}$ & $\mathrm{din}^{2}$ \\
\hline 刀 & 'knife' & $*_{-t}$ & ${ }^{*}-\mathrm{t}$ & $*_{-t}$ & $*_{-t}$ & $*-t$ & mat $^{7}$ & $\mathrm{mat}^{7}$ \\
\hline 屁 & 'fart' & $*-t$ & $*-t$ & $*-t$ & $*-t$ & ${ }^{*}-\mathrm{t}$ & $d \partial t^{7}$ & dut $^{7}$ \\
\hline 魚鱗 & 'fish scale' & ${ }^{*}$-c & $*_{-t}$ & ${ }^{*}$-c & - & $*-?$ & lip ${ }^{7}$ & $\operatorname{li} P^{7}$ \\
\hline 螞蟻 & 'ant' & ${ }^{*}-\mathrm{c}$ & $*_{-t}$ & ${ }^{*}-\mathrm{c}$ & $*-t$ & ${ }^{*}-1$ & mo? ${ }^{8}$ & mup $^{8}$ \\
\hline
\end{tabular}

\subsection{Closing Remarks}

Using the comparative method, the phonetic characteristics of proto-phonemes and associated details are reconstructible only when evidence survives. For instance, the voicing contrast in Proto-Ong-Be would be reconstructible only if at least one of today's Ong-Be varieties maintained such a contrast. Since none of the Ong-Be varieties surveyed in this paper reflect this, it is impossible to reconstruct a voicing contrast in proto initials. Reflexes in compensatory suprasegmental features, on the other hand, are attested, leading to my reconstruction of tone series associated with these initial consonants. Because modern Ong-Be languages do not allow consonant clusters anywhere, and all of the regular sound correspondences can be explained based only on the monosyllables that are permitted, no complex initials or complex syllable structures are reconstructed in this study. The results of my reconstruction and the structure of the contemporary varieties look alike, because few archaic features are preserved.

In my bottom-up reconstruction, Proto-Ong-Be finals include nasals $\left({ }^{*}-\mathrm{m},{ }^{*}-\mathrm{n}\right.$, and $\left.{ }^{*}-\mathrm{\eta}\right)$, glides $\left({ }^{*}\right.$-w and ${ }^{*}-\mathrm{j}$ ), and unreleased voiceless stops $\left({ }^{*}-\mathrm{p},{ }^{*}-\mathrm{t},{ }^{*}-\mathrm{k}\right.$, and $\left.{ }^{*}-\mathrm{P}\right)$, as demonstrated in Table 55 . These proto finals are well-preserved in contemporary Ong-Be varieties. Sporadic changes are observed, however. It is worth mentioning that the glottal stop in contemporary Ong-Be can reflect Proto-Kra-Dai *-c.

TABLE 55 Proto-Ong-Befinals

\begin{tabular}{|l|l|l|l|l|l|}
\hline & bilabial & alveolar & palatal & velar & glottal \\
\hline stops & $*-\mathrm{p}$ & $*_{-\mathrm{t}}$ & & $*-\mathrm{k}$ & $*-\mathrm{P}$ \\
\hline nasal & ${ }^{*}-\mathrm{m}$ & ${ }^{*}-\mathrm{n}$ & & $*-\mathrm{g}$ & \\
\hline approximants & ${ }^{*}-\mathrm{w}$ & & ${ }^{-}-\mathrm{j}$ & & \\
\hline
\end{tabular}

Table $5^{6}$ shows the mergers and splits of proto initials in selected daughter languages. ${ }^{*} \mathrm{z}^{1-}$ and ${ }^{*} \mathrm{z}^{2-}$ merged with ${ }^{*} \mathrm{z}^{1}$ - and ${ }^{*} \mathrm{z}^{2}$ - in the eastern subgroup, but with ${ }^{*} \mathrm{l}^{1}$ - and ${ }^{*} \mathrm{l}^{2}$ - in the western subgroup. The alternating reflexes observed in Huangtong (also see Table 23) are due to language contact, because the varieties to the east of Huangtong reflect ${ }^{*} \mathrm{z}^{1}$ - and ${ }^{*} \mathrm{z}^{2}$ - as $z$-, whereas the varieties to the west of it reflect these two proto initials as $l$-. In addition, ${ }^{*} \mathrm{n}^{1}$ - and ${ }^{*} \mathrm{n}^{2}$ - have merged with ${ }^{*} \mathrm{n}^{1}$ - and ${ }^{*} \mathrm{n}^{2}$ - in the eastern subgroup, while this distinction is maintained in most of the varieties of the western subgroup 
(excluding Xinying of Lingao and the Linshigang variety of Qiaotou I investigated). In Changliu, * $6^{1}$-, ${ }^{*} 6^{2}{ }^{-},{ }^{*} \mathrm{v}^{1}$-, and ${ }^{*} \mathrm{v}^{2}$ - have all merged as 6 -.

As for the bilabial and velar aspirated stops, they are fortified into an affricate or remain as an aspirated stop in Longtang and Changliu respectively, both of which belong to the eastern subgroup, but are spirantized as homorganic fricatives in most varieties in the western subgroup (except for Xinying, Meiliang, and Diaolou). Qiaotou Town in Chengmai County, which is located in the transition zone between the two subgroups, shows traits from each subgroup, even though it is classified as a member of the western subgroup.

\section{TABLE 56 Mergers in Eastern Ong-Be and Western Ong-Be varieties}

\begin{tabular}{|c|c|c|c|c|c|}
\hline Proto initial & LT & CL & LSG & нт & $\mathbf{X Y}$ \\
\hline${ }^{*} 6^{1-}$ & $/ 6^{1} /$ & $/ 6^{1} /$ & $/ 6^{1} /$ & $/ 6^{1} /$ & $/ 6^{1} /$ \\
\hline${ }^{*} b^{2-}$ & $\left|6^{2}\right|$ & $\left|6^{2}\right|$ & $\left|6^{2}\right|$ & $\mid 6^{2} /$ & $\left|6^{2}\right|$ \\
\hline${ }^{*} \mathbf{v}^{1}$ & $/ \mathrm{v}^{1} /$ & $/ 6^{1} /$ & $/ \mathbf{v}^{1} /$ & $/ \mathrm{v}^{1} /$ & $/ \mathrm{v}^{1} /$ \\
\hline${ }^{*} \mathbf{v}^{2}$ & $/ \mathrm{v}^{2} /$ & $\left|6^{2}\right|$ & $/ \mathrm{v}^{2} /$ & $/ \mathrm{v}^{2} /$ & $/ \mathrm{v}^{2} /$ \\
\hline *11- & $/ 1^{1 /}$ & $/ 1^{1} /$ & $/ \mathbf{l}^{1 /}$ & $/ 1^{1 /}$ & $/ 1^{1 /}$ \\
\hline${ }^{*} 1^{2}-$ & $/ 1^{2} /$ & $/ 1^{2} /$ & $/ 1^{2} /$ & $/ 1^{2} /$ & $/ 1^{2} /$ \\
\hline${ }^{*} \mathrm{z}^{1-}$ & $\mid \mathrm{z}^{1} /$ & $\left|\mathrm{z}^{1}\right|$ & $\mid z^{1}$ & $\left|\mathbf{z}^{1} / \sim / \mathbf{1}^{1}\right|$ & $/ 1 /$ \\
\hline${ }^{*} \mathrm{z}^{2-}$ & $\left|z^{2}\right|$ & $\left|\mathrm{z}^{2}\right|$ & $\left|\mathrm{z}^{2}\right|$ & $\left|\mathbf{z}^{2} / \sim / \mathbf{1}^{2}\right|$ & $/ 1 / 2$ \\
\hline${ }^{*} z^{1}-$ & $\left|\mathbf{z}^{1}\right|$ & $\left|\mathbf{z}^{1}\right|$ & $\left|\mathrm{z}^{1}\right|$ & $\left|z^{1}\right|$ & $\left|\mathbf{z}^{1}\right|$ \\
\hline${ }^{*} z^{2}-$ & $\left|\mathrm{z}^{2}\right|$ & $\left|\mathrm{z}^{2}\right|$ & $\left|\mathrm{z}^{2}\right|$ & $\left|z^{2}\right|$ & $\left|z^{2}\right|$ \\
\hline${ }^{*} n^{1-}$ & $/ \mathrm{n}^{1} /$ & $/ \mathrm{n}^{1 /}$ & $/ \mathrm{n}^{1} /$ & $/ \mathrm{n}^{1} /$ & $/ \mathrm{n}^{1} /$ \\
\hline${ }^{*} \mathbf{n}^{2}-$ & $/ \mathrm{n}^{2} /$ & $\left|\mathrm{n}^{2}\right|$ & $/ \mathrm{n}^{2} /$ & $/ \mathrm{n}^{2} /$ & $/ \mathrm{n}^{2} /$ \\
\hline${ }^{*} \mathrm{j}^{1-}$ & $/ \mathrm{n}^{1} /$ & $/ n^{1} /$ & $/ \mathrm{n}^{1} /$ & $/ \mathrm{n}^{1} /$ & $\left|z_{0}\right|$ \\
\hline${ }^{*} \mathrm{n}^{2-}$ & $/ \mathrm{n}^{2} /$ & $/ \mathrm{n}^{2} /$ & $/ \mathrm{n}^{2} /$ & $/ \mathrm{n}^{2} /$ & $\left|z^{2}\right|$ \\
\hline${ }^{*} \mathbf{p}^{\mathbf{h} \mathbf{H}_{-}}$ & $/ \mathrm{pf}^{1} /$ & $/ \mathrm{p}^{\mathrm{h} 1 /}$ & $/ \mathbf{f}^{1} /$ & $/ \mathbf{f}^{1} /$ & $/ \mathrm{p}^{\mathrm{h}} \mathrm{l}$ \\
\hline${ }^{*} \mathbf{p}^{\mathbf{h} 2_{-}}$ & $/ \mathrm{pf}^{2} /$ & $/ \mathrm{p}^{\mathrm{h} 2} /$ & $\left|\mathrm{f}^{2}\right|$ & $\left|\mathrm{f}^{2}\right|$ & $/ \mathrm{p}^{\mathrm{h} 2} /$ \\
\hline${ }^{*} \mathbf{k}^{\mathbf{h} 1}$ & $/ \mathrm{kx}^{1} /$ & $/ \mathrm{k}^{\mathrm{hl}} /$ & $/ x^{1} /$ & $/ x^{1} /$ & $/ \mathrm{k}^{\mathrm{h} 1} /$ \\
\hline${ }^{*} \mathbf{k}^{\mathbf{h} 2}$ & $/ \mathrm{kx}^{2} /$ & $/ \mathrm{k}^{\mathrm{h} 2} /$ & $\left|\mathrm{x}^{2}\right|$ & $\left|\mathrm{x}^{2}\right|$ & $/ \mathrm{k}^{\mathrm{h} 2} /$ \\
\hline
\end{tabular}

Through consulting external evidence, we are able to see how the Proto-Kra-Dai complex syllable canon became simplified in Ong-Be. An inverted reconstruction also uncovers details which the selected Ong-Be varieties have lost. The inverted reconstruction in Table 53 shows how Proto-Ong-Be phonemes are reflected accordingly in terms of voicing and their respective position in syllables. Based on internal evidence, none of these discoveries can be motivated because no evidence upon which to base a valid reconstruction is retained. 
A comparison of Ong-Be with external evidence also solves the mystery of why Ong-Be $t$ - and $d$-, both alveolar, took different sides in terms of voicing change, and it reveals the relative chronology of implosivization of alveolars and depalatalization of palatalized velars. However, the top-down approach raises a question as to whether the reconstructed result represents Proto-Ong-Be or the stage prior to the split of Proto-Ong-Be, i.e., pre-Proto-Ong-Be.

Sound changes from pre-Proto-Ong-Be to Proto-Ong-Be to modern Ong-Be are presented in Table 57, which shows that the most common mechanisms are spirantization and depalatalization. The other two mechanisms, fortition and lenition, are in complementary distribution. Note that this table does not include all the phonemes.

TABLE 57 From Proto-Kra-Dai to modern Ong-Be (not exhaustive)

\begin{tabular}{|c|c|c|c|c|c|c|}
\hline $\begin{array}{l}\text { PKD } \\
(\text { Ostapirat } 2005 \text { b) }\end{array}$ & $\begin{array}{l}\text { РОв (inverted) } \\
\text { (Ostapirat 2005b) }\end{array}$ & $\begin{array}{l}\text { Ров } \\
\text { (this study) }\end{array}$ & CL & LT & нт & $\mathrm{XY}$ \\
\hline *p- & *p & $* 6^{1}$ & $6^{1}$ & $6^{1}$ & $6^{1}$ & $6^{1}$ \\
\hline *b- & *b & $* 6^{2}$ & $6^{2}$ & $6^{2}$ & $6^{2}$ & $6^{2}$ \\
\hline${ }^{*} \mathrm{t}-$ & $* \mathrm{t}$ & ${ }^{*} d^{1}$ & $d^{1}$ & $d^{1}$ & $d^{1}$ & $d^{1}$ \\
\hline *d- & $* \mathrm{~d}$ & $* d^{2}$ & $d^{2}$ & $d^{2}$ & $d^{2}$ & $d^{2}$ \\
\hline${ }^{*} \mathrm{C}_{0}-\mathrm{KJ}_{-}-;{ }^{*} \mathrm{ts} \mathrm{j}_{-} ;{ }^{*} \mathrm{t}-$ & $*_{\mathrm{b}}$ & $* \mathrm{t}^{1}$ & $t^{1}$ & $t^{1}$ & $\mathrm{t}^{1}$ & $\mathrm{t}^{1}$ \\
\hline${ }^{*}-\mathrm{K}_{-}$ & $* \mathrm{~d}$ & $* \mathrm{t}^{2}$ & $t^{2}$ & $t^{2}$ & $t^{2}$ & $t^{2}$ \\
\hline *k- & ${ }^{*} \mathrm{k}$ & ${ }^{*} \mathrm{k}^{1}$ & $\mathrm{k}^{1}$ & $\mathrm{k}^{1}$ & $\mathrm{k}^{1}$ & $\mathrm{k}^{1}$ \\
\hline *-K- & $* g$ & $* \mathrm{k}^{2}$ & $\mathrm{k}^{2}$ & $\mathrm{k}^{2}$ & $\mathrm{k}^{2}$ & $\mathrm{k}^{2}$ \\
\hline${ }^{*} 3-;{ }^{*} \mathrm{k}-\mathrm{d} 3-$ & $* 3$ & ${ }^{*} z^{1}$ & $z^{1}$ & $z^{1}$ & $z^{1}$ & $z^{1}$ \\
\hline${ }^{*} \mathrm{k}-\mathrm{t}-;{ }^{*} \mathrm{~S}-$ & ${ }^{*} \mathrm{~h}$ & ${ }^{*} \mathrm{~h}^{1}$ & $h^{1}$ & $h^{1}$ & $h^{1}$ & $h^{1}$ \\
\hline${ }^{*} \mathrm{Pr}-$ or Kr- & *s & ${ }^{*} \mathrm{~s}^{1}$ & $s^{1}$ & $s^{1}$ & $s^{1}$ & $s^{1}$ \\
\hline${ }^{*} \mathrm{Br}-\mathrm{or}{ }^{*} \mathrm{Gr}-$ & $* \mathrm{~d}$ & $* \mathrm{ts}^{2}$ & $\mathrm{ts}^{2}$ & $\mathrm{ts}^{2}$ & $\mathrm{ts}^{2}$ & $\mathrm{ts}^{2}$ \\
\hline${ }^{*} \mathrm{C}-\mathrm{d}-$ & ${ }^{*} \mathrm{r}$ & ${ }^{*} \mathrm{z}^{1}$ & $z^{1}$ & $z^{1}$ & $l^{1}$ & $\mathrm{l}^{1}$ \\
\hline${ }^{*} \mathrm{C} / \mathrm{V}-\mathrm{d}-$ & $*^{r} \mathrm{r}$ & $* \mathrm{z}^{2}$ & $z^{2}$ & $z^{2}$ & $1^{2}$ & $1^{2}$ \\
\hline${ }^{*} \mathrm{C}-\mathrm{p}-$ & *v & ${ }^{*} \mathrm{v}^{1}$ & $6^{1}$ & $\mathrm{v}^{1}$ & $\mathrm{v}^{1}$ & $\mathrm{v}^{1}$ \\
\hline *-p- & *v & $* \mathrm{v}^{2}$ & $6^{2}$ & $\mathrm{v}^{2}$ & $\mathrm{v}^{2}$ & $\mathrm{v}^{2}$ \\
\hline *k-p- & *ky & ${ }^{*} \mathrm{p}^{\mathrm{h} 1}$ & $\mathrm{p}^{\mathrm{h} 1}$ & $\mathrm{pf}^{1}$ & $\mathrm{f}^{1}$ & $\mathrm{p}^{\mathrm{h} 1}$ \\
\hline${ }^{*} \mathrm{~K}-\mathrm{z}-$ & ${ }^{*} \mathrm{X}$ & ${ }^{*} \mathrm{k}^{\mathrm{hl}}$ & $\mathrm{k}^{\mathrm{h} 1}$ & $\mathrm{kx}^{1}$ & $x^{1}$ & $\mathrm{k}^{\mathrm{h} 1}$ \\
\hline $\mathrm{N} / \mathrm{A}$ & ${ }^{*} \mathrm{~V}$ & ${ }^{*} \mathrm{k}^{\mathrm{h} 2}$ & $\mathrm{k}^{\mathrm{h} 2}$ & $\mathrm{kx}^{2}$ & $x^{2}$ & $\mathrm{k}^{\mathrm{h} 2}$ \\
\hline
\end{tabular}




\section{Conclusion}

In this study, I have reconstructed a consonantal system for Proto-Ong-Be using the comparative method. 34 onsets (labelled with tone series) and nine codas are proposed, with the syllable structure of $\mathrm{CV}(:)(\mathrm{C})^{\mathrm{T}}$.

A summary of the Proto-Ong-Be initials and finals is given in Tables $5^{8-59}$.

table 58 Proto-Ong-Be initial inventory

\begin{tabular}{|c|c|c|c|c|c|c|c|c|c|c|}
\hline \multirow[b]{2}{*}{ implosives } & \multicolumn{2}{|c|}{ bilabial } & labiodental & \multicolumn{2}{|c|}{ alveolar } & \multicolumn{2}{|c|}{ alveopalatal } & \multicolumn{2}{|c|}{ velar } & \multirow[t]{2}{*}{ glottal } \\
\hline & ${ }^{*} 6^{1}$ & $* b^{2}$ & & ${ }^{*} d^{1}$ & ${ }^{*} d^{2}$ & & & & & \\
\hline stops & ${ }^{*} p^{h 1}$ & ${ }^{*} \mathrm{p}^{\mathrm{h} 2}$ & & ${ }^{*} \mathrm{t}^{1}$ & ${ }^{*} \mathrm{t}^{2}$ & & & $\begin{array}{l}{ }^{*} \mathrm{k}^{1} \\
{ }^{*} \mathrm{k}^{\mathrm{h}}\end{array}$ & $\begin{array}{l}{ }^{*} \mathrm{k}^{2} \\
{ }^{*} \mathrm{k}^{\mathrm{h}} 2\end{array}$ & ${ }^{*} P^{1}$ \\
\hline nasals & ${ }^{*} \mathrm{~m}^{1}$ & ${ }^{*} \mathrm{~m}^{2}$ & & ${ }^{*} n^{1}$ & ${ }^{*} \mathrm{n}^{2}$ & ${ }^{*} \mathrm{n}^{1}$ & ${ }^{*} \mathrm{n}^{2}$ & ${ }^{*} \mathrm{y}^{1}$ & $* \eta^{2}$ & \\
\hline fricatives & & & ${ }^{*} \mathrm{v}^{1} \quad{ }^{*} \mathrm{v}^{2}$ & $\begin{array}{l}{ }^{*} \mathrm{~s}^{1} \\
{ }^{*} \mathrm{z}^{1}\end{array}$ & ${ }^{*} \mathrm{z}^{2}$ & ${ }^{*} \mathrm{z}^{1}$ & ${ }^{*} \mathrm{z}^{2}$ & & & ${ }^{*} \mathrm{~h}^{1} \quad * \mathrm{~h}^{2}$ \\
\hline affricates & & & & $* t s^{1}$ & $* \mathrm{ts}^{2}$ & & & & & \\
\hline laterals & & & & $*^{1}$ & $* 1^{2}$ & & & & & \\
\hline
\end{tabular}

TABLE 59 Proto-Ong-Be finals

\begin{tabular}{|l|l|l|l|l|l|}
\hline & bilabial & alveolar & palatal & velar & glottal \\
\hline stops & ${ }^{*}-\mathrm{p}$ & ${ }^{*}-\mathrm{t}$ & & ${ }^{*}-\mathrm{k}$ & ${ }^{*}-\mathrm{l}$ \\
\hline nasals & ${ }^{*}-\mathrm{m}$ & ${ }^{*}-\mathrm{n}$ & & ${ }^{*}-\eta$ & \\
\hline glides & ${ }^{-}-\mathrm{w}$ & & ${ }^{*} \mathrm{j}$ & & \\
\hline
\end{tabular}

Proto-Ong-Be, which was monosyllabic and tonal, did not allow consonant clusters in onset or coda positions. The reconstruction of a Proto-Ong-Be consonantal system shows how Proto-Ong-Be fits in the broader Kra-Dai family. Most of the Proto-Kra-Dai finals retained in other Kra-Dai branches are also attested in Proto-Ong-Be. By contrast, compared with other Kra-Dai languages, which have preserved initial consonant clusters (a retention from disyllabic Proto-Kra-Dai), the phonology of Proto-Ong-Be is highly eroded. Initial consonant clusters cannot be reconstructed, and the earlier voicing contrast associated with initials cannot be distinguished using internal evidence.

To put it another way, while Ong-Be tones preserve information regarding earlier voicing contrasts, leading to my reconstruction of tone series 1 and 2, such information must be interpreted via comparisons from data beyond Ong-Be. Nevertheless, the indication of an earlier voicing contrast and the use of Kra-Dai tone categories are heuristic for discussing Ong-Be in a Kra-Dai context.

In addition, the syllable structure of Proto-Ong-Be can be explained using a monosyllabic model, unlike other phonologically conservative Kra-Dai languages, such as di- or sesquisyllabic Proto-Tai, Proto-Kam-Sui, Proto-Kra, and Proto-Hlai where a di- or sesquisyllabic model has a better explanatory function. 
This paper shows that the results inferred from a bottom-up approach and a top-down approach reflect different perspectives of Proto-Ong-Be consonants (reflecting different stages of the language), which should be interpreted as complementary not competing. This paper also shows that the gap between the results inferred from a bottom-up and a top-down approach cannot be filled in easily when no relevant information is retained. To conclude, based on the data gathered in the field, together with information from published materials, this study provides a clearer picture of the initials and finals of Ong-Be and Proto-Ong-Be.

\section{Acknowledgements}

I thank Chihkai Lin, Weera Ostapirat, and three anonymous reviewers for their valuable comments. I have benefited from my discussion with Paul Jen-kuei Li, Pei-chuan Wei, and Jackson T.-S. Sun during my visit at Academia Sinica, Taiwan. I am also grateful to the Henry Luce Foundation/ACLS Program and the Li Fang-Kuei Society for Chinese Linguistics for financial support which made my fieldwork trips possible. In addition, I would like to acknowledge my consultants, Li Guangliang (李光良), He Jingying (何京應), Xiao Aifeng (肖愛風), Wang Haixue (王海雪), Fu Houmin (符厚民), Fu Keqing (符克青), Fu Kecai (符克才), Chen Xingbian (陳興變), Zheng Fanghua (鄭方華), Wang Jianzhu (王建祝), Wang Shangyong (王尚勇), as well as Lin Biao (林表) and Fu Caizhi (符才之) at the Association of Cultures and Arts in Lingao (臨高文學藝術界聯合會) for their kind assistance in my data collection. I thank Andrew Pick for proofreading. All errors are my own.

\section{References}

Anttila, Raimo. 1989. Historical and Comparative Linguistics. Amsterdam/Philadelphia: John Benjamins Publishing Company.

Campbell, Lyle. 2013. Historical Linguistics: An Introduction (Third Edition). Edinburgh: Edinburgh University Press. Chen, Yen-ling. 2015. Obstruents in Proto-Ong-Be: A reconstruction. Paper presented at the $25^{\text {th }}$ Annual Meeting of the Southeast Asian Linguistics Society (SEALS), Chiang Mai, 27-29 May 2015.

Chen, Yen-ling. 2018. Proto-Ong-Be. Ph.D. dissertation, Honolulu: The University of Hawai' i at Mānoa.

Department of Chinese Literature (National Taiwan University) 臺灣大學中國文學系, \& Institute of Information Science (Academia Sinica) 中央研究院資訊科學研究所. n.d. 《漢字古今音資料庫》。(http://xiaoxue.iis .sinica.edu.tw/ccr/) (Accessed 2018-10-21.).

Edmondson, Jerold A., and David B. Solnit (eds.). 1997. Comparative Kadai: The Tai branch. Summer Institute of Linguistics Publications in Linguistics, 124. Dallas: Summer Institute of Linguistics and the University of Texas at Arlington.

Edmondson, Jerold A., and John H. Esling. 20o6. Valves of the throat and their functioning in tone, vocal register and stress: Laryngoscopic case studies. Phonology 23.2: 157-91.

Fox, Anthony. 1995. Linguistic Reconstruction: An Introduction to Theory and Method. New York: Oxford University Press.

Gedney, William J. 1989. Future directions in comparative Tai linguistics. Selected Papers on Comparative Tai Studies, eds. by Robert J. Bickner, John Hartmann, Thomas J. Hudak, and Patcharin Peyasantiwong, 7-116. Michigan: Center for South and Southeast Asian studies, the University of Michigan.

Hainan Administration of Surveying Mapping and Geoinformation 海南測繪地理信息局. 2017.《海南省全圖》。 (http://www.hbsm.gov.cn/wsfw_7045/bzdt/201702/t20170216_384724.html) (Accessed 2018-10-21). 
Hansell, Mark. 1988. The relationship of Be to Tai. Comparative Kadai: Linguistic Studies Beyond Tai, eds. by Jerold A. Edmondson and David B. Solnit, 239-287. Dallas: The Summer Institute of Linguistics and the University of Texas at Arlington.

Hashimoto, Mantaro. 1980. The Be Language: A Classified Lexicon of its Limkow Dialect. Tokyo: The Institute for the Study of Languages and Cultures of Asia and Africa.

Haudricourt, André-Georges. 1949. La conservation de la sonorité des sonores du thai commun dans le parler thô de Cao-bang. Actes du XxIe Congrès International des Orientalistes (Paris, 23-31 juillet 1948), 251-152. Paris: Société Asiatique.

Haudricourt, André-Georges. 1954a. De l'origine des tons en Vietnamien. Journal Asiatique 242: 69-82. English translation by Marc Brunelle: “The origin of tones in Vietnamese.” Evolution of Languages and Techniques (Trends in Linguistics. Studies and Monographs [TiLSM] 270), eds. by Martine Mazaudon, Alexis Michaud, and Boyd Michailovsky, in preparation. Berlin: De Gruyter Mouton. (https://halshs.archives-ouvertes.fr/halshs-01678018) (Accessed 2018-01-11).

Haudricourt, André-Georges. 1954b. Comment reconstruire le chinois archäque. Word 10, 2/3: 351-364. English translation by Guillaume Jacques: “How to reconstruct Old Chinese.” Evolution of languages and techniques (Trends in Linguistics. Studies and Monographs [TiLSM] 270), eds. by Martine Mazaudon, Alexis Michaud, and Boyd Michailovsky, in preparation. Berlin: De Gruyter Mouton. (https://halshs.archives-ouvertes.fr/ halshs-01631479) (Accessed 2018-01-11).

Haudricourt, André-Georges. 196o. Note sur les dialectes de la région de Moncay. Bulletin de l'Écolé Française d'Extrême-Orient 50: 167-177.

Haudricourt, André-Georges. 1961. Bipartition et tripartition des systèmes de tons dans quelques langues d'extrêmeOrient. Bulletin de La Société de Linguistique de Paris 56.1: 163-80. English translation by Court, Christopher. 1972. "Two-way and Three-way Splitting of Tonal Systems in Some Far Eastern Languages." Tai Phonetics and Phonology, eds. by Jimmy G. Harris and Richard B. Noss, 58-86. Bangkok: Central Institute of English Language, Mahidol University, 1972.

Hoàng, Văn Ma. 1997. The sound system of the Tày language of Cao Bằng Province, Vietnam. Comparative Kadai: The Tai Branch, eds. by Jerold A. Edmondson and David B. Solnit, 221-235. Dallas: Summer Institute of Linguistics and the University of Texas at Arlington.

Holiday China Tour. n.d. Map of Hainan. (http://www.holidaychinatour.com/pic/201272802959136.jpg) (Accessed 2018-10-21).

Jeremiassen, Carl C. 1893. Loi aborigines of Hainan and their speech. The China Review, or Notes \& Queries on the Far East 20.5: 296-305.

Li, Fang-kuei. 1977. A Handbook of Comparative Tai. Oceanic Linguistics Special Publication, No. 15. Honolulu: University Press of Hawaii.

Liang, Min 梁敏 and Zhang Junru 張均如. 1996.《侗台語族概論》。北京：中國社會科學出版社。 Liang, Min 梁敏 and Zhang Junru 張均如. 1997.《臨高語研究》。上海：上海遠東出版社。

Liu, Jiansan 劉劍三. 2000.《臨高漢詞典》。成都：四川民族出版社。

L-Thongkum, Theraphan. 1997. Implications of the retention of proto voiced obstruents in the Thu language of Yunnan for a theory of tonal development and Tai language classification. Comparative Kadai: The Tai Branch, eds. by Jerold A. Edmondson and David B. Solnit, 193-221. Dallas: Summer Institute of Linguistics and the University of Texas at Arlington.

Maddieson, Ian. 2013. Voicing and gaps in plosive systems. The World Atlas of Language Structures Online, eds. by Matthew S. Dryer and Martin Haspelmath. (http://wals.info/chapter/5) (Accessed 2018-04-13).

Meakins, Felicity. 2013. Mixed languages. Contact Languages, eds. by Yaron Matras and Peter Bakker, 165-236. Berlin: Mouton. 
Michaud, Alexis. 2012. Monosyllabicization: patterns of evolution in Asian languages. Monosyllables: from phonology to typology, Nicole Nau, Thomas Stolz \& Cornelia Stroh (eds.), 115-130. Berlin: Akademie Verlag.

Norquest, Peter K. 2007. A Phonological Reconstruction of Proto-Hlai. Ph.D. dissertation, Tucson: University of Arizona.

Norquest, Peter K. 2015. A Phonological reconstruction of Proto-Hlai. Leiden and Boston: Brill.

Ostapirat, Weera. 1998. A mainland Bê language? Journal of Chinese Linguistics 26.2: 338-344.

Ostapirat, Weera. 2000. Proto-Kra. Linguistics of the Tibeto-Burman Area 23.1:1-251.

Ostapirat, Weera. 2004. Proto-Hlai sound system and lexicons. Studies on Sino-Tibetan Languages: Papers in Honor of Professor Hwang-cherng Gong on His $70^{\text {th }}$ Birthday, eds. by Ying-chin Lin (林英津), Fang-min Hsu (徐芳敏), Chun-chih Lee (李存智), Jackson T.-S. Sun (孫天心), Hsiu-fang Yang (楊秀芳), and Dah-an Ho (何大安), 121175. Taipei: Academia Sinica.

Ostapirat, Weera. 2005a. Tai-Kadai and Austronesian: Notes on phonological correspondences and vocabulary distribution. The Peopling of East Asia: Putting Together Archaeology, Linguistics and Genetics, eds. by Laurent Sagart, Roger Blench and A. Sanchez-Mazas, 109-133. London: RoutledgeCurzon.

Ostapirat, Weera. 2005b. Be's obstruent consonants in Kra-Dai perspective. POLA Forever: Festschrift in Honor of Professor William S.-Y. Wang on His $70^{\text {th }}$ Birthday, eds. by Dah-an Ho (何大安) and Ovid J.L. Tzeng (曾志朗), 275-292. Taipei: Institute of Linguistics, Academia Sinica.

Ostapirat, Weera. 2009. Proto-Tai and Kra-Dai final *-1 and *-c. Journal of Language and Culture, 28.2: 41-56.

Ostapirat, Weera. 2016. Sino-Tai Hypothesis. Encyclopedia of Chinese Language and Linguistics, eds. by Rint Sybesma, Wolfgang Behr, Yueguo Gu, Zev Handel, C.-T. James Huang, and James Myers, 127-133. Leiden \& Boston: Brill.

Ostapirat, Weera. 2017. The Peopling of Kra-Dai in South China. Paper presented at the International Conference on the Ancestry and of Languages and Peoples of China, Guangzhou, 30-31 May 2017.

Parker, Edward Harper. 1892. The Li aboriginese of K'iung Shan. The China Review, or Notes \& Queries on the Far East 19.6.

Pittayaporn, Pittayawat. 2009. The Phonology of Proto-Tai. Ph.D. dissertation, Ithaca: Cornell University.

Savina, François Marie. 1965. Le Vocabulaire Bê de F.M. Savina (Présente par André-Georges Haudricourt). Paris: École française d'Extrême-Orient. [in French].

Solnit, David B. 1982. Linguistic contact in ancient south China: The case of Hainan Chinese, Be, and Vietnamese. Proceedings of the Eighth Annual Meeting of the Berkeley Linguistics Society, 219-230. (http://linguistics.berkeley .edu/bls/previous_proceedings/bls8.pdf) (Accessed 2015-10-07).

Stübel, Hans. 1937. Die Li-stämme der insel Hainan. Berlin: Klinkhardt \& Biermann [in German].

The Institute of Linguistics (Chinese Academy of Social Sciences) 中國社會科學院語言研究所, the Institute of Ethnology and Anthropology (Chinese Academy of Social Sciences) 中國社會科學院民族學與人類學研究所, \& the Language Information Sciences Research Centre (City University of Hong Kong) 香港城市大學語言資訊 科學研究中心 (eds). 2012. 《中國語言地圖集》。北京：商務印書館。

Thurgood, Graham. 1988. Notes on the reconstruction of Proto-Kam-Sui. Comparative Kadai Linguistic Studies Beyond Tai, eds. by Jerold A. Edmondson and David B. Solnit, 179-218. Summer Institute of Linguistics Publications in Linguistics, No. 86. Dallas: Summer Institute of Linguistics and the University of Texas at Arlington.

Woon, Wee-Lee. 1979. A synchronic phonology of Hainan dialect: Part II. Journal of Chinese Linguistics 7.2: 268-302.

Xin, Shibiao 辛世彪. 2006. 〈臨高語的特殊音變及其與同族語言的關係〉。《東方語言學》1:214-245.

Xin, Shibiao 辛世彪. 2007. 〈十九世紀傳教士紀錄的海南臨高語〉。《語言研究》27.4: 115-124.

Xin, Shibiao 辛世彪. 2008.〈海口臨高語長流方言〉。《民族語文》2008.2: 24-36.

Xin, Shibiao 辛世虎. 2011. 〈臨高語第二調在海口石山方言中的促化〉。《廣西民族大學學報》33.2: 34-39.

Zhang, Yuansheng 張元生, Ma Jialin 馬加林, Wen Mingying 文明英, Wei Xinglang 韋星朗. 1985.《海南臨高 話》。南寧：廣西民族出版社。 


\section{原始臨高話的聲母和韻尾}

陳彥伶

夏威夷大學/ 中央研究院

yenling@hawaii.edu

\section{摘要}

本文運用「歷史比較法」構擬原始臨高話的聲母和韻尾。有鑑於各方言點聲調皆分陰陽，本研究認為原始 臨高話的聲母已無清濁對立。而臨高語群韻尾一致性甚高, 透過「歷史比較法」可如實反映早期的韻尾系 統。本文同時討論各家擬音異同之成因。

\section{關鍵詞}

臨高話、聲母、韻尾、構擬、侗台語 